April 19, 2018

\title{
Blown-up intersection cohomology
}

\author{
David Chataur, Martintxo Saralegi-Aranguren, and Daniel Tanré
}

\begin{abstract}
In previous works, we have introduced the blown-up intersection cohomology and used it to extend Sullivan's minimal models theory to the framework of pseudomanifolds, and to give a positive answer to a conjecture of M. Goresky and W. Pardon on Steenrod squares in intersection homology.

In this paper, we establish the main properties of this cohomology. One of its major feature is the existence of cap and cup products for any filtered space and any commutative ring of coefficients, at the cochain level. Moreover, we show that each stratified map induces an homomorphism between the blown-up intersection cohomologies, compatible with the cup and cap products. We prove also its topological invariance in the case of a pseudomanifold with no codimension one strata. Finally, we compare it with the intersection cohomology studied by G. Friedman and J.E. McClure.

A great part of our results involves general perversities, defined independently on each stratum, and a tame intersection homology adapted to large perversities.
\end{abstract}

\section{Contents}

Introduction

Part 1. Blown-up intersection cohomology. Stratified maps

1. Some reminders.

2. Blown-up complex of a weighted simplicial complex. 6

3. Blown-up intersection cohomology. 11

4. Cup product. 13

5. Intersection and tame intersection (co)homology. $\quad 15$

6. Cap product. $\quad 16$

7. Stratified maps: the local level.

8. Stratified maps: the global level. Theorem A

Part 2. Properties of the Blown-up intersection cohomology 27

9. $\mathcal{U}$-small chains. TheoremB 27

10. Mayer-Vietoris exact sequence. Theorem C. 36

11. Product with the real line. Theorem D. 37

12. Blown-up intersection cohomology of a cone. Theorem E 42

13. Comparison between intersection cohomologies. Theorem $\mathrm{F}$.

14. Topological invariance. Theorem G. 53

15. Decomposition of the ordinary cap product. 55

References

2010 Mathematics Subject Classification. 55N33, 57N80, 55S05.

Key words and phrases. Intersection cohomology, Cap product; Cup product;Topological invariance.

The third author is partially supported by the MINECO and FEDER research project MTM2016-78647-P and ANR-11-LABX-0007-01 "CEMPI". 


\section{Introduction}

Intersection homology was defined by M. Goresky and R. MacPherson in the case of PL-pseudomanifolds in [16] and with sheaf theory in [17] where the authors restore Poincaré duality for pseudomanifolds: if $X$ is a compact oriented pseudomanifold of dimension $n$ and $\bar{p}, \bar{q}$ are two complementary perversities, there is an isomorphism

$$
H_{r}^{\bar{p}}(X ; \mathbb{Q}) \cong H_{\bar{q}}^{n-r}(X ; \mathbb{Q}),
$$

with $H_{\bar{q}}^{n-r}(X ; \mathbb{Q}):=\operatorname{hom}\left(H_{n-r}^{\bar{q}}(X ; \mathbb{Q}) ; \mathbb{Q}\right)$. This restoration also presents some disadvantages :

(1) If we don't restrict ourselves to perversities comprised between the zero $\overline{0}$ and the top $\bar{t}$ perversities respecting some growth condition, then we don't have Poincaré duality anymore and we need to use the more general tame intersection cohomology $\mathfrak{H}_{\bar{p}}^{*}(X ; R)$ of $X$ (see Definition [5.5),

(2) The cohomology is defined via a linear dual process and not via a geometrical one,

(3) This duality is not satisfied for any commutative ring $R$. Especially for a commutative ring of positive characteristic properties of "torsion freeness" for the links of the singularities are needed.

Blown-up intersection cohomology aims to overcome some of these difficulties. The goal in this article is to develop the main properties of the Blown-up intersection cohomology $\mathscr{H}_{\bar{p}}^{*}(X ; R)$ for filtered spaces and more precisely for CS sets. This theory was successfully used in [4] to extend the theory of Sullivan's minimal models for intersection cohomology and in [8] to answer a conjecture on Steenrod squares.

The first sections contain some reminders about the type of stratified spaces and maps used in the rest of the paper. After that come the definitions by a local system of the blown-up complex $\widetilde{N}_{\bar{p}}^{*}(X ; R)$, first for a regular simplex, then by a limit argument for a general weighted simplicial complex in Proposition 2.8 and finally for a perverse space $(X, \bar{p})$ in Definition 3.2 . The interesting features are that

(1) the perversities $\bar{p}$ used here are completely general, lifting the restriction on the perversities used for classical intersection cohomology,

(2) it is well defined for any commutative ring $R$ as coefficients ring, regardless of its characteristic.

The existence and properties of a cup product for this cohomology are proved in Proposition 4.2 by the existence of an associative multiplication

$$
-\cup-: \tilde{N}_{\bar{p}}^{k}(X ; R) \otimes \tilde{N}_{\bar{q}}^{\ell}(X ; R) \rightarrow \tilde{N}_{\bar{p}+\bar{q}}^{\ell+k}(X ; R),
$$

inducing a graded commutative multiplication with unity

$$
-\cup-: \mathscr{H}_{\bar{p}}^{k}(X ; R) \otimes \mathscr{H}_{\bar{q}}^{\ell}(X ; R) \rightarrow \mathscr{H}_{\bar{p}+\bar{q}}^{\ell+k}(X ; R) .
$$

The same goes for the cap product, proving in Proposition 6.7 that the cap product allows an interaction between the blown-up intersection cohomology $\mathscr{H}_{\bar{p}}^{*}(X ; R)$ and the tame intersection homology $\mathfrak{H}_{*}^{\bar{p}}(X ; R)$.

The first part ends with two sections detailing the behavior of a stratified map with respect to blown-up intersection cohomology, first at the local level and then 
globally. The key result being here the Theorem $₫$ stating that any stratified map $f: X \rightarrow Y$ as defined in the Definition 1.5 induces a chain map

$$
f^{*}: \widetilde{N}_{\bar{p}}^{k}(Y ; R) \rightarrow \tilde{N}_{\bar{p}}^{k}(X ; R)
$$

compatible with the cup product. This theorem also states that the induced maps $f_{*}$ and $f^{*}$ are compatible with the interaction between the blown-up intersection cohomology $\mathscr{H}_{\bar{p}}^{*}(X ; R)$ and the tame intersection homology $\mathfrak{H}_{*}^{\bar{p}}(X ; R)$ of Proposition 6.7 .

The second part of the paper is devoted to the various properties of the blownup intersection cohomology and their proofs. The first property comes from the Theorem $\mathrm{B}$ stating the existence of the complex of $\mathcal{U}$-small chains $\tilde{N}_{\bar{p}}^{*, \mathcal{U}}(X, R)$ where $\mathcal{U}$ is an open cover of the perverse space $(X, \bar{p})$ and the existence of a quasiisomorphism $\tilde{N}_{\bar{p}}^{*}(X, R) \rightarrow \tilde{N}_{\bar{p}}^{*, \mathcal{U}}(X, R)$. This leads then to the Theorem $\mathrm{C}$ where the Mayer- Vietoris exact sequence is proved. The two next sections detail results about explicit computations with the blown-up intersection cohomology, the first one is the Theorem $\mathrm{D}$ determining the blown-up intersection cohomology of the product of a filtered space $X$ with the line $\mathbb{R}$, from the existence of an isomorphism

$$
\mathscr{H}_{\bar{p}}^{*}(X \times \mathbb{R} ; R) \cong \mathscr{H}_{\bar{p}}^{*}(X ; R) .
$$

The second result is the Theorem $\mathrm{E}$ giving the computation for the blown-up intersection cohomology of an open cone $\stackrel{\circ}{\mathrm{C}}$ over a compact filtered space $X$.

The paper finishes with the two following theorems. The Theorem $\mathrm{F}$ compares the blown-up intersection cohomology $\mathscr{H}_{\bar{p}}^{*}(X ; R)$ to the already known tame intersection cohomology $\mathfrak{H}_{\bar{p}}^{*}(X ; R)$ for $(X, \bar{p})$ a paracompact separable perverse CS set and proves that if $R$ is a field or if the space $X$ is a locally $(D \bar{p}, R)$-torsion free pseudomanifold there is an isomorphism

$$
\mathscr{H}_{\bar{p}}^{*}(X ; R) \cong \mathfrak{H}_{D \bar{p}}^{*}(X ; R)
$$

where $D \bar{p}$ is the complementary perversity. The last Theorem G states that the blown-up intersection cohomology is a topological invariant when working with GMperversities and separable paracompact CS sets with no codimension one strata .

We fix for the sequel a commutative ring $R$ with unity. All (co)homologies in this work are considered with coefficients in $R$. For a topological space $X$, we denote by $\mathrm{c} X=X \times[0,1] / X \times\{0\}$ the cone on $X$ and $\stackrel{\circ}{\mathrm{c}}=X \times[0,1[/ X \times\{0\}$ the open cone on $X$.

We thank the anonymous referee for her/his comments and suggestions which have in particular contributed to improve the writing and the organization of this introduction.

\section{Part 1. Blown-up intersection cohomology. Stratified maps}

We introduce the main notion of this work: the blown-up intersection cohomology and its associated cup and cap products. 


\section{Some reminders.}

This section contains the basic definitions and properties of the main notions used in this work.

Definition 1.1. A filtered space is a Hausdorff topological space endowed with a filtration by closed sub-spaces

$$
\varnothing=X_{-1} \subseteq X_{0} \subseteq X_{1} \subseteq \ldots \subseteq X_{n-1} \varsubsetneqq X_{n}=X .
$$

The formal dimension of $X$ is $\operatorname{dim} X=n$.

The non-empty connected components of $X_{i} \backslash X_{i-1}$ are the strata of $X$. Those of $X_{n} \backslash X_{n-1}$ are regular strata, while the others are singular strata. The family of strata of $X$ is denoted by $\mathcal{S}_{X}$. The singular set is $X_{n-1}$, denoted by $\Sigma_{X}$ or simply $\Sigma$. The formal dimension of a stratum $S \subset X_{i} \backslash X_{i-1}$ is $\operatorname{dim} S=i$. The formal codimension of $S$ is $\operatorname{codim} S=\operatorname{dim} X-\operatorname{dim} S$.

An open subset $U$ of $X$ can be provided with the induced filtration, defined by $U_{i}=U \cap X_{i}$. If $M$ is a topological manifold, the product filtration is defined by $(M \times X)_{i}=M \times X_{i}$ (see remarks about shifted filtrations of subsections 3.1 and 5.1).

The more restrictive concept of stratified space provides a better behavior of the intersection (co)homology with regard to continuous maps.

Definition 1.2. A stratified space is a filtered space verifying the following frontier condition: for any two strata $S, S^{\prime} \in \mathcal{S}_{X}$ such that $S \cap \overline{S^{\prime}} \neq \varnothing$ then $S \subset \overline{S^{\prime}}$.

In their work ([16, [17), Goresky and MacPherson proved that the intersection (co)homology has richer properties on a singular space $X$ with local conical structure: these are the classical stratified pseudomanifolds. The local structure is characterized by the fact that any stratum $S$ is a manifold having a conical transversal structure over the link $L$. This link must be in turn a compact stratified pseudomanifold. Friedman [14] observed that we can suppose the link $L$ to be only a compact filtered space to preserve the (co)homological properties of $X$. These are the CS sets of L. Siebenman, [25.

Definition 1.3. A $C S$ set of dimension $n$ is a filtered space,

$$
\varnothing \subset X_{0} \subseteq X_{1} \subseteq \cdots \subseteq X_{n-2} \subseteq X_{n-1} \varsubsetneqq X_{n}=X,
$$

such that, for each $i, X_{i} \backslash X_{i-1}$ is a topological manifold of dimension $i$ or the empty set. Moreover, for each point $x \in X_{i} \backslash X_{i-1}, i \neq n$, there exist

(i) an open neighborhood $V$ of $x$ in $X$, endowed with the induced filtration,

(ii) an open neighborhood $U$ of $x$ in $X_{i} \backslash X_{i-1}$,

(iii) a compact filtered space $L$, with formal dimension $n-i-1$, where the open

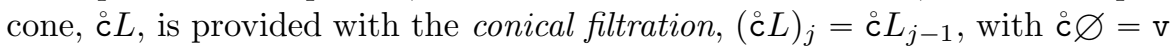
the apex of $\stackrel{\circ}{c} L$,

(iv) a homeomorphism, $\varphi: U \times \stackrel{\circ}{c} L \rightarrow V$, such that

(a) $\varphi(u, \mathrm{v})=u$, for each $u \in U$,

(b) $\varphi\left(U \times \stackrel{\circ}{\mathrm{c}} L_{j}\right)=V \cap X_{i+j+1}$, for each $j \in\{0, \ldots, n-i-1\}$.

The pair $(V, \varphi)$ is a conical chart of $x$ and the filtered space $L$ is a link of $x$. The CS set is called normal if the links are connected. 
In the above definition, the links are always non-empty sets. Therefore, the open subset $X_{n} \backslash X_{n-1}$ is dense. Links are not necessarily CS sets but they are always filtered spaces. Note also that the links associated to points living in the same stratum may be not homeomorphic but they always have the same intersection homology, see for example [14, Chapter 5]. Finally, note that any open subset of a CS set is a CS set, that any CS set is a locally compact stratified space 4, Theorem G] and that a paracompact CS set is metrizable [5. Proposition 1.11].

The pseudomanifolds are special cases of CS sets. Their definition varies in the literature; we consider here the original definition of M. Goresky and R. MacPherson [16.

DeFINITION 1.4. An n-dimensional pseudomanifold (or simply pseudomanifold) is an $n$-dimensional CS set, where the link $L$ of a point $x \in X_{i} \backslash X_{i-1}$ is an $(n-i-1)$-dimensional pseudomanifold. We refer to a pseudomanifold such that $X_{n-1}=X_{n-2}$ as a classical $n$-dimensional pseudomanifold .

There are several notions of stratified maps.

Definition 1.5. Let $f: X \rightarrow Y$ be a continuous map between two stratified spaces. The map $f$ is a stratified map, if it sends a stratum $S$ of $X$ in a stratum $S^{f}$ of $Y, f(S) \subset S^{f}$, verifying $\operatorname{codim} S \geqslant \operatorname{codim} S^{f}$.

The stratified map $f$ is a stratified homeomorphism, if $f$ and $f^{-1}$ are stratified maps.

The map $f$ is a stratum preserving stratified map if $n=\operatorname{dim} X=\operatorname{dim} Y$ and $f^{-1}\left(Y_{n-\ell}\right)=X_{n-\ell}$, for any $\ell \in\{0, \ldots, n\}$.

This notion of stratified map does not match exactly with those found in [14] or 17 .

There are two families of perversities: filtration depending (introduced by Goresky-MacPherson [16]) and the stratification depending (introduced by MacPherson [22]).

Definition 1.6. A Goresky-MacPherson perversity (or GM-perversity) (see 16]) is a map $\bar{p}: \mathbb{N} \rightarrow \mathbb{Z}$, verifying $\bar{p}(0)=\bar{p}(1)=\bar{p}(2)=0$ and $\bar{p}(i) \leqslant \bar{p}(i+1) \leqslant$ $\bar{p}(i)+1$ for each $i \geqslant 2$.

We use in this work the notion of perversity introduced by MacPherson [22] and also present in 23, 24, 12, 11, 13, 15. Unlike classic perversities, these perversities are not maps depending only on the codimension of the strata but are maps defined on the strata themselves.

Definition 1.7. A perversity on a filtered space $X$ is a map $\bar{p}: \mathcal{S}_{X} \rightarrow \mathbb{Z} \cup\{ \pm \infty\}$ taking the value 0 on the regular strata. The pair $(X, \bar{p})$ is called a perverse space. If $X$ is a CS set, we will say that $(X, \bar{p})$ is a perverse $C S$ set.

The top perversity is the perversity defined by $\bar{t}(S)=\operatorname{codim}(S)-2$ on singular strata. The complementary perversity of a perversity $\bar{p}$ is the perversity $D \bar{p}=\bar{t}-\bar{p}$. The zero perversity is defined by $\overline{0}(S)=0$.

A GM-perversity induces a perversity on a filtered space $X$, still denoted by $\bar{p}$, defined by $\bar{p}(S)=\bar{p}(\operatorname{codim} S)$.

Definition 1.8. Let $f: X \rightarrow Y$ be a stratified map. The pull-back of a perversity $\bar{q}$ of $Y$ is the perversity $f^{*} \bar{q}$ of $X$ defined by $f^{*} \bar{q}(S)=\bar{q}\left(S^{f}\right)$. 


\section{Blown-up complex of a weighted simplicial complex.}

The blown-up complex is based on the blow up of a filtered simplex. This technique goes back to [2], (see also [24]). In this section, we introduce the notions and first properties associated to the blow up of a regular euclidean simplex (see Definition 2.2) and, more generally, of a weighted simplicial complex (see Definition 2.1).

The cone of an euclidean simplex $\Delta=\left[e_{0}, \ldots, e_{m}\right]$ is the simplicial simplex $\mathrm{c} \Delta=\left[e_{0}, \ldots, e_{m}, \mathrm{v}\right]$.

The notation $\nabla \triangleleft \Delta$ means that $\nabla$ is a face of $\Delta$.

2.1. Differential complexes associated to a simplicial complex. Let $\mathcal{L}$ be an oriented simplicial complex whose family of vertices is $\mathcal{V}(\mathcal{L})=\left\{e_{0}, \ldots, e_{m}\right\}$. We denote $\left(N_{*}(\mathcal{L}), \partial\right)$ the $R$-complex of linear simplices of $\mathcal{L}$. The differential of $\left(N_{*}(\mathcal{L}), \partial\right)$ is defined by

$$
\partial\left[e_{i_{0}}, \ldots, e_{i_{p}}\right]=\sum_{k=0}^{p}(-1)^{k}\left[e_{i_{0}}, \ldots, \hat{e}_{i_{k}}, \ldots, e_{i_{p}}\right] .
$$

For any vertex $e \in \mathcal{V}(\mathcal{L})$, we define the homomorphism - *e: $N_{p}(\mathcal{L}) \rightarrow N_{p+1}(\mathcal{L})$, by

$$
\left[e_{i_{0}}, \ldots, e_{i_{p}}\right] * e=\left\{\begin{array}{cl}
{\left[e_{i_{0}}, \ldots, e_{i_{p}}, e\right]} & \text { if }\left[e_{i_{0}}, \ldots, e_{i_{p}}, e\right] \subset \mathcal{L}, \\
0 & \text { if not. }
\end{array}\right.
$$

This operator is extended to the empty set by $\varnothing * e=[e]$. When $\left[e_{0}, \ldots, e_{r}\right]$ does not match an ordered simplex, by convention, it means $(-1)^{\varepsilon(\sigma)}\left[e_{\sigma(0)}, \ldots, e_{\sigma(r)}\right]$, where $\sigma$ is the permutation for which $\left[e_{\sigma(0)}, \ldots, e_{\sigma(r)}\right]$ is an ordered simplex and $\varepsilon(\sigma)$ its signature. We also put $\left[e_{0}, \ldots, e_{r}\right]=0$ if two vertices $e_{i}$ are equal.

Let $\left(N^{*}(\mathcal{L}), \delta\right)$ be the $R$-dual, $\operatorname{hom}\left(N_{*}(\mathcal{L}), R\right)$, equipped with the transpose differential of $\partial$, defined by $(\delta f)(v)=-(-1)^{|f|} f(\partial v)$. Among the elements of $N^{*}(\mathcal{L})$, we consider the dual basis of the simplices of $\mathcal{L}$; i.e., if $F$ is a $p$-simplex of $\mathcal{L}$, we denote by $\mathbf{1}_{F}$ the $p$-cochain taking the value 1 on $F$ and 0 on the other simplices of $\mathcal{L}$. If $e \in \mathcal{V}(\mathcal{L})$, we also introduce a homomorphism, $-* e: N^{p}(\mathcal{L}) \rightarrow N^{p+1}(\mathcal{L})$, defined by

$$
\mathbf{1}_{\left[e_{i_{0}}, \ldots, e_{i_{p}}\right]} * e=(-1)^{p} \mathbf{1}_{\left[e_{i_{0}}, \ldots, e_{i_{p}}, e\right]},
$$

putting $\mathbf{1}_{\left[e_{i_{0}}, \ldots, e_{i_{p}} e\right]}=0$ if $\left[e_{i_{0}}, \ldots, e_{i_{p}}, e\right] \not \mathcal{L}$. On the elements of the dual basis, the differential $\delta: N^{*}(\mathcal{L}) \rightarrow N^{*+1}(\mathcal{L})$ takes the value $\delta \mathbf{1}_{\left[e_{i_{0}}, \ldots, e_{i_{p}}\right]}=\sum_{k \notin\left\{i_{0}, \ldots, i_{p}\right\}} \mathbf{1}_{\left[e_{i_{0}}, \ldots, e_{i_{p}}, e_{k}\right]}$. Using $\left[e_{i_{0}}, \ldots, e_{i_{k}}\right]=0$ when two of the vertices are equal, we can consider that the above sum is indexed by the set of vertices of $\mathcal{L}$; i.e.,

$$
\delta \mathbf{1}_{\left[e_{i_{0}}, \ldots, e_{i_{p}}\right]}=\sum_{e \in \mathcal{V}(\mathcal{L})} \mathbf{1}_{\left[e_{i_{0}}, \ldots, e_{i_{p}}, e\right]}=(-1)^{p} \sum_{e \in \mathcal{V}(\mathcal{L})} \mathbf{1}_{\left[e_{i_{0}}, \ldots, e_{i_{p}}\right]} * e .
$$

The cochain $\delta \mathbf{1}_{F}$ depends on the simplicial complex $\mathcal{L}$ in which the face $F$ lives. We denote by $\delta^{\mathcal{L}} \mathbf{1}_{F}$ the differential of $\mathbf{1}_{F}$ in $\mathcal{L}$, when necessary.

DeFinition 2.1. A weighted simplicial complex is an oriented simplicial complex, where each vertex $e$ has a weight $w(e) \in\{0, \ldots, n\}$. The integer $n$ is called the formal dimension of $\mathcal{L}$. 
Let $\mathcal{L}_{i}$ be the union of the simplices of $\mathcal{L}$ whose vertices have a weight equal to $i$. Any simplex $F$ of $\mathcal{L}$ is written (modulo orientation) as a join, $F=F_{0} * \cdots * F_{n}$, with $F_{i} \subset \mathcal{L}_{i}$. They are the filtered euclidean simplices.

Let $\mathrm{c} \mathcal{L}_{i}$ be the cone on $\mathcal{L}_{i}$, whose apex $\mathrm{v}_{i}$ is called virtual vertex, chosen as the last vertex of the cone; i.e., if $F_{i}=\left[e_{i_{0}}, \ldots, e_{i_{p}}\right] \subset \mathcal{L}_{i}$, then $c F_{i}=\left[e_{i_{0}}, \ldots, e_{i_{p}}, \mathrm{v}_{i}\right]$. The face $\left[\mathrm{v}_{i}\right]$ can be considered as the cone on the empty set. For a comprehensive treatment of all these cases, we consider the empty set as a simplex of $\mathcal{L}_{i}$.

The full blow up of a weighted simplicial complex $\mathcal{L}$ is the prismatic set $\widetilde{\mathcal{L}}^{\text {all }}=$ c $\mathcal{L}_{0} \times \cdots \times$ c $\mathcal{L}_{n}$. For each $i \in\{0, \ldots, n\}$, we denote by $L_{i}$ - a simplex $F_{i}$ of $\mathcal{L}_{i}$ - or the cone $\mathrm{c} F_{i}$ of a simplex of $\mathcal{L}_{i}$,- either the singleton reduced to the virtual vertex $\mathrm{v}_{i}$. The products $L_{0} \times \cdots \times L_{n}$ are called faces of $\widetilde{\mathcal{L}}^{\text {all }}$ and represented by

$$
(F, \varepsilon)=\left(F_{0}, \varepsilon_{0}\right) \times \cdots \times\left(F_{n}, \varepsilon_{n}\right),
$$

satisfying the following conventions:

- the iterated join, $F=F_{0} * \cdots * F_{n}$, is a simplex of $\mathcal{L}$;

- if $\varepsilon_{i}=0$ and $F_{i} \neq \varnothing$, then $\left(F_{i}, 0\right)=F_{i}$ is a simplex of $\mathcal{L}_{i}$;

- if $\varepsilon_{i}=1$ and $F_{i} \neq \varnothing$, then $\left(F_{i}, 1\right)=\mathrm{c} F_{i}$ is the cone over the simplex $F_{i}$ of $\mathcal{L}_{i}$;

- if $F_{i}=\varnothing$, one must have $\varepsilon_{i}=1$.

Let $(F, \varepsilon)=\left(F_{0}, \varepsilon_{0}\right) \times \cdots \times\left(F_{n}, \varepsilon_{n}\right)$ be a face of $\tilde{\mathcal{L}}^{\text {all }}$ and let $\gamma \in\{0, \ldots, n\}$. We put,

- $|(F, \varepsilon)|_{<\gamma}=\sum_{i<\gamma}\left(\operatorname{dim} F_{i}+\varepsilon_{i}\right)$ and $|(F, \varepsilon)|_{>\gamma}=\sum_{i>\gamma}\left(\operatorname{dim} F_{i}+\varepsilon_{i}\right)$,

- $|(F, \varepsilon)|_{\leqslant \gamma}=\sum_{i \leqslant \gamma}\left(\operatorname{dim} F_{i}+\varepsilon_{i}\right)$ and $|(F, \varepsilon)|_{\geqslant \gamma}=\sum_{i \geqslant \gamma}\left(\operatorname{dim} F_{i}+\varepsilon_{i}\right)$,

- $|(F, \varepsilon)|=|(F, \varepsilon)|_{\leqslant n}$.

Definition 2.2. The blow up of a weighted simplicial complex of dimension $n, \mathcal{L}$, is the sub-prism $\widetilde{\mathcal{L}}$ of $\widetilde{\mathcal{L}}^{\text {all }}$ consisting of the $(F, \varepsilon)^{\prime} s$ such that $\varepsilon_{n}=0$. Notice that the corresponding simplices, $F=F_{0} * \cdots * F_{n} \subset \mathcal{L}$, must verify $F_{n} \neq \varnothing$. This kind of filtered simplices are called regular.

EXAMPLE 2.3. The generic case of a weighted simplicial complex is a regular simplex $\Delta=\Delta_{0} * \cdots * \Delta_{n}$. Assume $\Delta$ is oriented by an order on its vertices, $\left(e_{i}\right)_{0 \leqslant i \leqslant m}, e_{i}<e_{i+1}$, in a compatible way with the join decomposition. More precisely, expressing the maximal simplex by its vertices, and indicating by a vertical bar the filtration change, we have

$$
\Delta=[\underbrace{e_{0}, \ldots, e_{k_{0}}}_{\Delta_{0}}|\underbrace{e_{k_{0}+1}, \ldots, e_{k_{1}}}_{\Delta_{1}}| \cdots|\underbrace{\varnothing}_{\Delta_{i}}| \cdots \mid \underbrace{e_{k_{n-1}+1}, \ldots, e_{k_{n}}}_{\Delta_{n}}],
$$

with $k_{n}=m$. The vertices $\left\{e_{0}, \ldots, e_{k_{\ell}}\right\}$ generate the simplex $\Delta_{0} * \cdots * \Delta_{\ell}$. A face $\Delta_{i}$ can be empty, as shown in the decomposition above. As we have already noticed, giving the filtration $\Delta=\Delta_{0} * \cdots * \Delta_{n}$ of the simplex $\Delta$ is equivalent to giving a weight on each vertex of $\Delta$, in the sense of Definition 2.1. The corresponding blow up is

$$
\widetilde{\Delta}=\mathrm{c} \Delta_{0} \times \cdots \times \mathrm{c} \Delta_{n-1} \times \Delta_{n} .
$$

EXAMPLE 2.4. The coding of the faces of the blow up of a regular simplex under the form of a product $(F, \varepsilon)=\left(F_{0}, \varepsilon_{0}\right) \times \cdots \times\left(F_{n}, \varepsilon_{n}\right)$ is used throughout the text. To familiarize the reader with this representation, we specify the blow up $\widetilde{\Delta}$ of $\Delta=\Delta_{0} * \Delta_{1}=\left[e_{0}\right] *\left[e_{1}, e_{2}\right]$. 


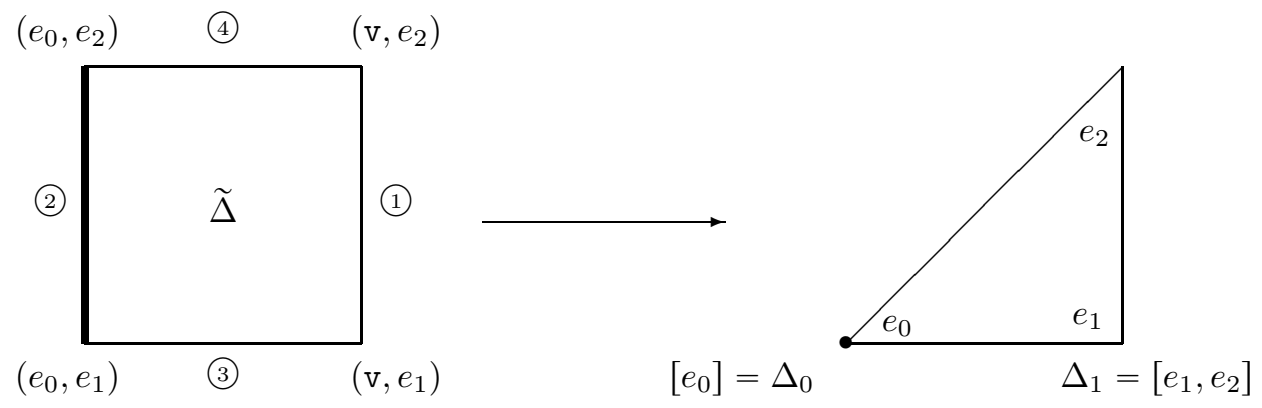

The four one dimensional faces of the blow up $\widetilde{\Delta}=\mathrm{c} \Delta_{0} \times \Delta_{1}$ are encoded as

$$
\begin{array}{ll}
\text { (1) }=(\varnothing, 1) \times\left(\Delta_{1}, 0\right), & (2)=\left(\left[e_{0}\right], 0\right) \times\left(\Delta_{1}, 0\right), \\
\text { (3) }=\left(\left[e_{0}\right], 1\right) \times\left(\left[e_{1}\right], 0\right), & (4)=\left(\left[e_{0}\right], 1\right) \times\left(\left[e_{2}\right], 0\right),
\end{array}
$$

corresponding to

$$
\text { (1) }=[\mathrm{v}] \times \Delta_{1}, \quad \text { (2) }=\Delta_{0} \times \Delta_{1}, \quad \text { (3) }=\mathrm{c} \Delta_{0} \times\left[e_{1}\right], \quad \text { (4) }=\mathrm{c} \Delta_{0} \times\left[e_{2}\right] \text {. }
$$

2.2. Blown-up complex associated to a weighted simplicial complex. We recall the notations of the previous paragraph. The element $\mathbf{1}_{\left(F_{i}, \varepsilon_{i}\right)}$ is the cochain on $\mathrm{c} \mathcal{L}_{i}$, taking the value 1 on the simplex $\left(F_{i}, \varepsilon_{i}\right)$ and 0 on other simplices of $c \mathcal{L}_{i}$. If $F=F_{0} * \cdots * F_{n} \subset \mathcal{L}$ and $(F, \varepsilon)=\left(F_{0}, \varepsilon_{0}\right) \times \cdots \times\left(F_{n}, \varepsilon_{n}\right)$, we write

$$
\mathbf{1}_{(F, \varepsilon)}=\mathbf{1}_{\left(F_{0}, \varepsilon_{0}\right)} \otimes \cdots \otimes \mathbf{1}_{\left(F_{n}, \varepsilon_{n}\right)} .
$$

The $R$-module generated by these elements when $F$ runs over the simplices of $\mathcal{L}$ is denoted by $\widetilde{N}^{a l l, *}(\mathcal{L} ; R)$ or $\widetilde{N}^{a l l, *}(\mathcal{L})$ if there is no ambiguity. The sub- $R$-module generated by the elements such that $\varepsilon_{n}=0$, endowed with the differential

$$
\begin{aligned}
\delta \mathbf{1}_{(F, \varepsilon)}= & \sum_{i=0}^{n-1}(-1)^{|(F, \varepsilon)|_{<i}} \mathbf{1}_{\left(F_{0}, \varepsilon_{0}\right)} \otimes \cdots \otimes \delta^{c \mathcal{L}_{i}} \mathbf{1}_{\left(F_{i}, \varepsilon_{i}\right)} \otimes \cdots \otimes \mathbf{1}_{F_{n}} \\
& +(-1)^{|(F, \varepsilon)|_{<n}} \mathbf{1}_{\left(F_{0}, \varepsilon_{0}\right)} \otimes \cdots \otimes \mathbf{1}_{\left(F_{n-1}, \varepsilon_{n-1}\right)} \otimes \delta^{\mathcal{L}_{n}} \mathbf{1}_{F_{n}},
\end{aligned}
$$

is called the blown-up complex of $\mathcal{L}$ and denoted by $\tilde{N}^{*}(\mathcal{L} ; R)$ or simply $\tilde{N}^{*}(\mathcal{L})$. (Recall that $\delta^{\mathrm{c} \mathcal{L}_{i}}$ is the differential of the cochain complex on the simplicial complex c $\mathcal{L}_{i}$.)

The elements of the blown-up complex are provided with an additional degree which looks like the degree of a differential form along the fiber in a bundle.

Definition 2.5. Let $\ell \in\{1, \ldots, n\}$. The $\ell$-perverse degree of the cochain $\mathbf{1}_{(F, \varepsilon)}$ is equal to

$$
\left\|\mathbf{1}_{(F, \varepsilon)}\right\|_{\ell}=\left\{\begin{array}{ccc}
-\infty & \text { if } & \varepsilon_{n-\ell}=1 \\
|(F, \varepsilon)|_{>n-\ell} & \text { if } & \varepsilon_{n-\ell}=0
\end{array}\right.
$$


If $\omega \in \tilde{N}^{*}(\mathcal{L} ; R)$ decomposes as $\omega=\sum_{\mu} \lambda_{\mu} \mathbf{1}_{\left(F_{\mu} \varepsilon_{\mu}\right)}$, with $\lambda_{\mu} \neq 0$, its $\ell$-perverse degree is equal to

$$
\|\omega\|_{\ell}=\max _{\mu}\left\|\mathbf{1}_{\left(F_{\mu}, \varepsilon_{\mu}\right)}\right\|_{\ell} .
$$

By convention, we set $\|0\|_{\ell}=-\infty$.

EXAMPLE 2.6. We compute the perverse degree of some cochains of the blow up $\widetilde{\Delta}=c \Delta_{0} \times c \Delta_{1} \times \Delta_{2}$ of the regular simplex $\Delta=\Delta_{0} * \Delta_{1} * \Delta_{2}$.
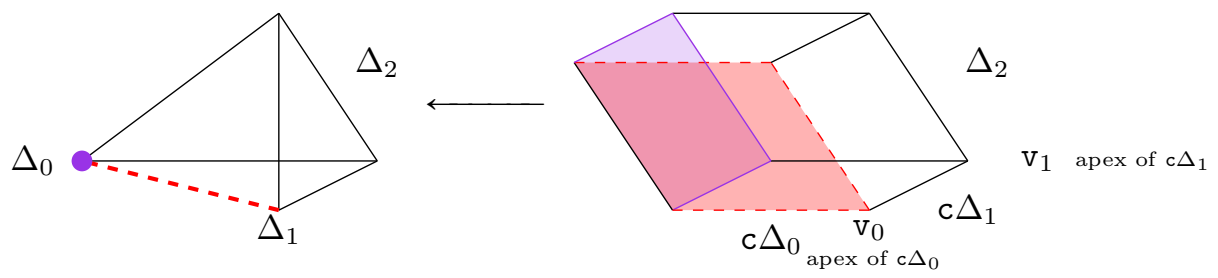

$$
\begin{array}{ll}
\left\|\mathbf{1}_{\mathrm{v}_{0} \times \mathrm{v}_{1} \times \Delta_{2}}\right\|_{2}=-\infty & \left\|\mathbf{1}_{\mathrm{v}_{0} \times \mathrm{v}_{1} \times \Delta_{2}}\right\|_{1}=-\infty \\
\left\|\mathbf{1}_{c \Delta_{0} \times \Delta_{1} \times \Delta_{2}}\right\|_{2}=-\infty & \left\|\mathbf{1}_{c \Delta_{0} \times \Delta_{1} \times \Delta_{2}}\right\|_{1}=\operatorname{dim} \Delta_{2} \\
\left\|\mathbf{1}_{\Delta_{0} \times \mathrm{c} \Delta_{1} \times \Delta_{2}}\right\|_{2}=\operatorname{dim} \mathrm{c} \Delta_{1}+\operatorname{dim} \Delta_{2} & \left\|\mathbf{1}_{\Delta_{0} \times \mathrm{c} \Delta_{1} \times \Delta_{2}}\right\|_{1}=-\infty \\
\left\|\mathbf{1}_{\Delta_{0} \times \Delta_{1} \times \Delta_{2}}\right\|_{2}=\operatorname{dim} \Delta_{1}+\operatorname{dim} \Delta_{2} & \left\|\mathbf{1}_{\Delta_{0} \times \Delta_{1} \times \Delta_{2}}\right\|_{1}=\operatorname{dim} \Delta_{2}
\end{array}
$$

(We have used $\mathrm{v}_{i}$ the apex of the cone $\mathrm{c} \Delta_{i}, i=0,1$ ).

DeFinition 2.7. In the case of a regular simplex $\Delta=\Delta_{0} * \cdots * \Delta_{n}, \Delta_{n} \neq \varnothing$, the blown-up complex is the tensor product, $\tilde{N}^{*}(\Delta)=N^{*}\left(\mathrm{c} \Delta_{0}\right) \otimes \cdots \otimes N^{*}\left(\mathrm{c} \Delta_{n-1}\right) \otimes$ $N^{*}\left(\Delta_{n}\right)$ which corresponds to [4, Definition 1.31].

The face operators of a filtered simplex $\Delta=\Delta_{0} * \cdots * \Delta_{n}$ are maps $\mu: \Delta^{\prime}=$ $\Delta_{0}^{\prime} * \cdots * \Delta_{n}^{\prime} \rightarrow \Delta=\Delta_{0} * \cdots * \Delta_{n}$, of the form $\mu=*_{i=0}^{n} \mu_{i}$ where $\mu_{i}$ is an injective map preserving the order. The face $\Delta^{\prime}$ is a codimension 1 regular face. Each operator face of a factor of the join $\delta_{\ell}: \Delta_{i}^{\prime} \rightarrow \Delta_{i}$ induces a map, still denoted by $\delta_{\ell}: N_{*}\left(\mathrm{c} \Delta_{0}^{\prime}\right) \otimes \cdots \otimes N_{*}\left(\mathrm{c} \Delta_{n-1}^{\prime}\right) \otimes N_{*}\left(\Delta_{n}^{\prime}\right) \rightarrow N_{*}\left(\mathrm{c} \Delta_{0}\right) \otimes \cdots \otimes N_{*}\left(\mathrm{c} \Delta_{i}\right) \otimes \cdots \otimes$ $N_{*}\left(\Delta_{n}\right)$, obtained by carrying out the join with the identity map on factors $\Delta_{j}$, $j \neq \ell$. We call $\delta_{\ell}^{*}: N^{*}\left(\mathrm{c} \Delta_{0}\right) \otimes \cdots \otimes N^{*}\left(\mathrm{c} \Delta_{n-1}\right) \otimes N^{*}\left(\Delta_{n}\right) \rightarrow N^{*}\left(\mathrm{c} \Delta_{0}^{\prime}\right) \otimes \cdots \otimes$ $N^{*}\left(\mathrm{c} \Delta_{i}^{\prime}\right) \otimes \cdots \otimes N^{*}\left(\Delta_{n}^{\prime}\right)$, the transpose of the linear map $\delta_{\ell}$.

The blown-up complex of a weighted simplicial complex is expressed from the blown-up complex of regular simplices.

Proposition 2.8. Let $\mathcal{L}$ be a weighted simplicial complex. Then

$$
\tilde{N}^{*}(\mathcal{L} ; R) \cong \lim _{\Delta \subset \mathcal{L}, \overleftarrow{\Delta \text { regular }}} \tilde{N}^{*}(\Delta ; R)
$$

Proof. An element $\omega$ of $\lim _{\longleftarrow} \widetilde{N}^{*}(\Delta)$ is a family $\omega=\left(\omega_{\Delta}\right)_{\Delta}$, indexed by the regular simplices of $\mathcal{L}$, with $\omega_{\Delta} \in \widetilde{N}^{*}(\Delta)$ and satisfying the following face compatibility conditions: if $\delta_{\ell}: \nabla \rightarrow \Delta$ is a regular face of codimension 1 of a simplex $\Delta$ of $\mathcal{L}$, then $\delta_{\ell}^{*} \omega_{\Delta}=\omega_{\nabla} \in \tilde{N}^{*}(\nabla)$. To $\omega \in \varliminf_{\longleftarrow} \widetilde{N}^{*}(\Delta)$, we associate the cochain $\omega_{\mathcal{L}} \in \tilde{N}^{k}(\mathcal{L})$ defined by

$$
\omega_{\mathcal{L}}(F, \varepsilon)=\omega_{F}(F, \varepsilon)
$$


Conversely, let $\omega_{\mathcal{L}}=\sum_{F} \alpha_{(F, \varepsilon)} \mathbf{1}_{(F, \varepsilon)} \in \tilde{N}^{k}(\mathcal{L})$, where $F$ runs over the regular simplices of $\mathcal{L},|(F, \varepsilon)|=k$ and $\alpha_{(F, \varepsilon)} \in R$. For each regular simplex $\Delta$ of $\mathcal{L}$, we define $\omega_{\Delta} \in \widetilde{N}^{k}(\Delta)$ by

$$
\omega_{\Delta}=\sum_{F \triangleleft \Delta} \alpha_{(F, \varepsilon)} \mathbf{1}_{(F, \varepsilon)} .
$$

It remains to prove the compatibility with respect to the face operators. Let $\delta_{\ell}: \nabla \rightarrow \Delta$ be a regular face of $\Delta$ and $F$ a simplex of $\Delta$. By definition of the dual basis one has

$$
\delta_{\ell}^{*}\left(\mathbf{1}_{(F, \varepsilon)}\right)=\left\{\begin{array}{cl}
\mathbf{1}_{(F, \varepsilon)} & \text { if } F \subset \nabla \\
0 & \text { if not. }
\end{array}\right.
$$

It follows

$$
\delta_{\ell}^{*}\left(\omega_{\Delta}\right)=\sum_{F \triangleleft \nabla} \alpha_{(F, \varepsilon)} \mathbf{1}_{(F, \varepsilon)}=\omega_{\nabla}
$$

\subsection{Adjunction of a vertex to a cochain of the blow up.}

Definition 2.9. Let $(F, \varepsilon)=\left(F_{0}, \varepsilon_{0}\right) \times \cdots \times\left(F_{n}, \varepsilon_{n}\right)$ be a face of $\tilde{\mathcal{L}}^{\text {all }}$ and $\ell \in\{0, \ldots, n\}$. The adjunction of a vertex $e \in \mathcal{L}_{\ell}$ to the cochain $\mathbf{1}_{(F, \varepsilon)}$ is the cochain

$$
\mathbf{1}_{(F, \varepsilon)} * e=(-1)^{|(F, \varepsilon)|_{>\ell}} \mathbf{1}_{\left(F_{0}, \varepsilon_{0}\right)} \otimes \cdots \otimes\left(\mathbf{1}_{\left(F_{\ell}, \varepsilon_{\ell}\right)} * e\right) \otimes \cdots \otimes \mathbf{1}_{\left(F_{n}, \varepsilon_{n}\right)} .
$$

Likewise, for the virtual vertex $v_{\ell}$, we set,

$$
\mathbf{1}_{(F, \varepsilon)} * \mathrm{v}_{\ell}=(-1)^{|(F, \varepsilon)|>\ell} \mathbf{1}_{\left(F_{0}, \varepsilon_{0}\right)} \otimes \cdots \otimes\left(\mathbf{1}_{\left(F_{\ell}, \varepsilon_{\ell}\right)} * \mathrm{v}_{\ell}\right) \otimes \cdots \otimes \mathbf{1}_{\left(F_{n}, \varepsilon_{n}\right)} .
$$

We extend by linearity this adjunction to $\tilde{N}^{\text {all,* }}(\mathcal{L} ; R)$.

The next property follows directly from the definition.

LeMma 2.10. Let $\mathcal{L}$ be a weighted simplicial complex and $(F, \varepsilon)$ a face of $\widetilde{\mathcal{L}}^{\text {all }}$. Consider two vertices of $\mathcal{L}, e_{\alpha} \in \mathcal{L}_{\ell(\alpha)}$ and $e_{\beta} \in \mathcal{L}_{\ell(\beta)}$, and two virtual vertices $\mathrm{v}_{\ell}$ and $\mathrm{v}_{\ell^{\prime}}$. Then the following properties are verified,

$$
\begin{aligned}
\mathbf{1}_{(F, \varepsilon)} * e_{\alpha} * e_{\beta} & =-\mathbf{1}_{(F, \varepsilon)} * e_{\beta} * e_{\alpha}, \\
\mathbf{1}_{(F, \varepsilon)} * e_{\alpha} * \mathrm{v}_{\ell} & =-\mathbf{1}_{(F, \varepsilon)} * \mathrm{v}_{\ell} * e_{\alpha}, \\
\mathbf{1}_{(F, \varepsilon)} * \mathrm{v}_{\ell} * \mathrm{v}_{\ell^{\prime}} & =-\mathbf{1}_{(F, \varepsilon)} * \mathrm{v}_{\ell^{\prime}} * \mathrm{v}_{\ell} .
\end{aligned}
$$

Proposition 2.11. Let $\mathcal{L}$ be a weighted simplicial complex. The blown-up differential of an element $\mathbf{1}_{(F, \varepsilon)} \in \tilde{N}^{*}(\mathcal{L} ; R)$ can be written as,

$$
\delta \mathbf{1}_{(F, \varepsilon)}=(-1)^{|(F, \varepsilon)|}\left(\sum_{e \in \mathcal{V}(\mathcal{L})} \mathbf{1}_{(F, \varepsilon)} * e+\sum_{i=0}^{n-1} \mathbf{1}_{(F, \varepsilon)} * \mathrm{v}_{i}\right) .
$$

Proof. Let $\mathbf{1}_{(F, \varepsilon)}=\mathbf{1}_{\left(F_{0}, \varepsilon_{0}\right)} \otimes \cdots \otimes \mathbf{1}_{\left(F_{n-1}, \varepsilon_{n-1}\right)} \otimes \mathbf{1}_{F_{n}}$. By replacing $\delta^{\text {c } \mathcal{L}_{i}}$ and $\delta^{\mathcal{L}_{n}}$ by their values from (2.1) in the equality (2.4) and denoting $\left|\left(F_{i}, \varepsilon_{i}\right)\right|=$ 
$\operatorname{dim} F_{i}+\varepsilon_{i}$, we get,

$$
\begin{array}{r}
\sum_{i=0}^{n}(-1)^{|(F, \varepsilon)|<i} \sum_{e_{i} \in \mathcal{V}\left(\mathcal{L}_{i}\right)}(-1)^{\left|\left(F_{i}, \varepsilon_{i}\right)\right|} \mathbf{1}_{\left(F_{0}, \varepsilon_{0}\right)} \otimes \cdots \otimes\left(\mathbf{1}_{\left(F_{i}, \varepsilon_{i}\right)} * e_{i}\right) \otimes \cdots \otimes \mathbf{1}_{\left(F_{n}, \varepsilon_{n}\right)} \\
+\sum_{i=0}^{n-1}(-1)^{|(F, \varepsilon)| \leqslant i} \mathbf{1}_{\left(F_{0}, \varepsilon_{0}\right)} \otimes \cdots \otimes\left(\mathbf{1}_{\left(F_{i}, \varepsilon_{i}\right)} * \mathrm{v}_{i}\right) \otimes \cdots \otimes \mathbf{1}_{F_{n}} .
\end{array}
$$

The wanted formula follows from the definition of the adjunction of a vertex.

From Proposition 2.11 and Lemma 2.10, we directly deduce the behavior of the adjunction of a vertex with respect to the differential of the blown-up complex.

Corollary 2.12. Let $\mathcal{L}$ be a weighted simplicial complex and let $\mathbf{1}_{(F, \varepsilon)} \in$ $\tilde{N}^{*}(\mathcal{L} ; R)$. For each vertex $e_{\alpha} \in \mathcal{V}(\mathcal{L})$ and each virtual vertex $\mathrm{v}_{\ell}$, one has $\delta\left(\mathbf{1}_{(F, \varepsilon)} *\right.$ $\left.e_{\alpha}\right)=\left(\delta \mathbf{1}_{(F, \varepsilon)}\right) * e_{\alpha}$ and $\delta\left(\mathbf{1}_{(F, \varepsilon)} * \mathrm{v}_{\ell}\right)=\left(\delta \mathbf{1}_{(F, \varepsilon)}\right) * \mathrm{v}_{\ell}$.

\section{Blown-up intersection cohomology.}

In this section, we define the blown-up intersection cohomology of a perverse space.

Definition 3.1. Let $X$ be a filtered space. A filtered singular simplex is a continuous map, $\sigma: \Delta \rightarrow X$, where the euclidean simplex $\Delta$ is endowed with a decomposition $\Delta=\Delta_{0} * \Delta_{1} * \cdots * \Delta_{n}$, called $\sigma$-decomposition of $\Delta$, verifying

$$
\sigma^{-1} X_{i}=\Delta_{0} * \Delta_{1} * \cdots * \Delta_{i}
$$

for each $i \in\{0, \ldots, n\}$. The filtered singular simplex $\sigma$ is regular if $\Delta_{n} \neq \varnothing$. To specify that the filtration of the euclidean simplex $\Delta$ is induced from that of $X$ by the map $\sigma$, we sometimes write $\Delta=\Delta_{\sigma}$. This notation is particularly useful when a simplex carries two filtrations associated with two distinct maps.

The dimensions of the simplices $\Delta_{i}$ of the $\sigma$-decomposition measure the nontransversality of the simplex $\sigma$ with respect to the strata of $X$. These simplices $\Delta_{i}$ may be empty, with the convention $\varnothing * Y=Y$, for any space $Y$. Note also that a singular simplex $\sigma: \Delta \rightarrow X$ is filtered if each $\sigma^{-1}\left(X_{i}\right), i \in\{0, \ldots, n\}$, is a face of $\Delta$.

To any regular simplex, $\sigma: \Delta=\Delta_{0} * \cdots * \Delta_{n} \rightarrow X$, we associate the cochain complex defined by

$$
\tilde{N}_{\sigma}^{*}=\tilde{N}^{*}(\Delta)=N^{*}\left(\mathrm{c} \Delta_{0}\right) \otimes \cdots \otimes N^{*}\left(\mathrm{c} \Delta_{n-1}\right) \otimes N^{*}\left(\Delta_{n}\right) .
$$

If $\delta_{\ell}: \Delta^{\prime}=\Delta_{0}^{\prime} * \cdots * \Delta_{\ell}^{\prime} * \cdots * \Delta_{n}^{\prime} \rightarrow \Delta=\Delta_{0} * \cdots * \Delta_{\ell} * \cdots * \Delta_{n}$ is a face operator, we denote $\partial_{\ell} \sigma$ the filtered simplex defined by $\partial_{\ell} \sigma=\sigma \circ \delta_{\ell}: \Delta^{\prime} \rightarrow X$. The face operator $\delta_{\ell}$ is regular if $\Delta_{n}^{\prime} \neq \varnothing$.

Definition 3.2. Let $X$ be a filtered space. The blown-up complex of $X$ with coefficients in $R, \tilde{N}^{*}(X ; R)$, is the cochain complex formed by the elements $\omega$, associating to any regular filtered simplex, $\sigma: \Delta_{0} * \cdots * \Delta_{n} \rightarrow X$, an element $\omega_{\sigma} \in \widetilde{N}_{\sigma}^{*}$, so that $\delta_{\ell}^{*}\left(\omega_{\sigma}\right)=\omega_{\partial_{\ell} \sigma}$, for any regular face operator, $\delta_{\ell}$. The differential $\delta \omega$ of $\omega \in \tilde{N}^{*}(X ; R)$ is defined by $(\delta \omega)_{\sigma}=\delta\left(\omega_{\sigma}\right)$ for any regular filtered simplex $\sigma$. 
For any $\ell \in\{1, \ldots, n\}$, the element $\omega_{\sigma} \in \tilde{N}_{\sigma}^{*}$ is endowed with the perverse degree $\left\|\omega_{\sigma}\right\|_{\ell}$, introduced in Definition 2.5. We extend this degree to the elements of $\tilde{N}^{*}(X ; R)$ as follows.

Definition 3.3. Let $X$ be a filtered space and $\omega \in \tilde{N}^{*}(X ; R)$. The perverse degree of $\omega$ along a singular stratum, $S$, is equal to

$$
\|\omega\|_{S}=\sup \left\{\left\|\omega_{\sigma}\right\|_{\operatorname{codim} S} \mid \sigma: \Delta \rightarrow X \text { regular with } \sigma(\Delta) \cap S \neq \varnothing\right\} .
$$

We denote by $\|\omega\|$ the map associating to any singular stratum $S$ of $X$ the element $\|\omega\|_{S}$ and 0 to a regular stratum.

Notice that, by definition, face operators $\delta_{\ell}^{*}$ decrease the perverse degree.

Definition 3.4. Let $(X, \bar{p})$ be a perverse space. A cochain $\omega \in \tilde{N}^{*}(X ; R)$ is $\bar{p}$-allowable if

$$
\|\omega\| \leqslant \bar{p}
$$

A cochain $\omega$ is a $\bar{p}$-intersection cochain if $\omega$ and its coboundary, $\delta \omega$, are $\bar{p}$ allowable. We denote by $\tilde{N}_{\bar{p}}^{*}(X ; R)$ the complex of $\bar{p}$-intersection cochains and $\mathscr{H}_{*}^{\bar{p}}(X ; R)$ its homology, called blown-up intersection cohomology of $X$ with coefficients in $R$, for the perversity $\bar{p}$.

3.1. Shifted filtrations. Blown-up intersection cohomology does not depend on the dimension of the strata of $X$ but on the codimension of these strata (see [14. Section 4.3.1]). Let us consider on $X$ the shifted filtration $Y$, where $m \in \mathbb{N}^{*}$ :

$$
\begin{array}{r}
\varnothing=Y_{0}=\cdots=Y_{m-1} \subset Y_{m}=X_{0} \subset \cdots \subset Y_{n+m-1}= \\
X_{n-1} \subset Y_{n+m}=X_{n}=X .
\end{array}
$$

So, the formal dimension of $Y$ is $n+m$. For example, on $\mathbb{R}^{m} \times X$ we have two natural shifted filtrations: $\left(\mathbb{R}^{m} \times X\right)_{i}=\mathbb{R}^{m} \times X_{i}$, with $i \in\{0, \ldots, n\}$, and $\left(\mathbb{R}^{m} \times X\right)_{i}=$ $\mathbb{R}^{m} \times X_{i-m}$, with $i \in\{0, \ldots, n+m\}$.

The family of regular simplices is the same for both filtrations. The perverse degree of a filtered simplex $\sigma: \Delta \rightarrow X=Y$ is the same for both filtrations. Let us see that. If $S$ is a singular stratum of $X$ (and therefore of $Y$ ) with $\operatorname{Im} \sigma \cap$ $S \neq \varnothing$, we have $\operatorname{codim}_{X} S=\operatorname{codim}_{Y} S$. Let $\ell$ be this codimension. On the other hand, if $\Delta_{\sigma}^{X}=\Delta_{0} * \cdots * \Delta_{n}$ is the induced filtration by $\sigma: \Delta \rightarrow X$ then $\Delta_{\sigma}^{Y}=\underbrace{\varnothing * \cdots * \varnothing}_{m \text { times }} * \Delta_{m}^{\prime} * \cdots * \Delta_{n+m}^{\prime}$, with $\Delta_{m+r}^{\prime}=\Delta_{r}$, is the induced filtration by $\sigma: \Delta \rightarrow Y$. So, if $\left(F=F_{0} * \cdots * F_{n}, \varepsilon=\left(\varepsilon_{0} * \cdots * \varepsilon_{n}\right)\right)$ is a face of the blow up of $\Delta_{\sigma}^{X}$ then $(F^{\prime}=\underbrace{\varnothing, \ldots, \varnothing}_{m \text { times }} * F_{0} * \cdots * F_{n}, \varepsilon^{\prime}=(\underbrace{1, \ldots, 1}_{m \text { times }}, \varepsilon_{0}, \ldots, \varepsilon_{n}))$ is the corresponding face of the blow up of $\Delta_{\sigma}^{Y}$. We have, for $\ell \in\{1, \ldots, n\}$

$$
\begin{aligned}
\left\|\mathbf{1}_{(F, \varepsilon)}\right\|_{\ell}^{X} & =\left\{\begin{array}{ll}
-\infty & \text { if } \varepsilon_{n-\ell}=1 \\
|(F, \varepsilon)|_{>n-\ell} & \text { if } \varepsilon_{n-\ell}=0
\end{array}= \begin{cases}-\infty & \text { if } \varepsilon_{m+n-\ell}^{\prime}=1 \\
\left|\left(F^{\prime}, \varepsilon^{\prime}\right)\right|_{>m+n-\ell} & \text { if } \varepsilon_{m+n-\ell}^{\prime}=0\end{cases} \right. \\
& =\| \mathbf{1}_{\left(F^{\prime}, \varepsilon^{\prime}\right) \|_{\ell}^{Y},}
\end{aligned}
$$

and $\left\|\mathbf{1}_{\left(F^{\prime}, \varepsilon^{\prime}\right)}\right\|_{\ell}^{Y}=-\infty \quad$ if $\ell \in\{n+1, \ldots, m+n\}$. We get $\tilde{N}_{\bar{p}}^{*}(X ; R)=\tilde{N}_{\bar{p}}^{*}(Y ; R)$ and therefore $\mathscr{H}_{\bar{p}}^{*}(X ; R)=\mathscr{H}_{\bar{p}}^{*}(Y ; R)$. 
We prove in Theorem A that any stratified map induces a morphism in blownup intersection cohomology. The following Proposition, which is a weaker version of this result, is used in Part 2

Proposition 3.5. Let $f: X \rightarrow Y$ be a stratum preserving stratified map. The induced map $f: \widetilde{N}^{*}(Y ; R) \rightarrow \widetilde{N}^{*}(X ; R)$, defined by $\left(f^{*}(\omega)\right)_{\sigma}=\omega_{f \circ \sigma}$, is a well defined chain map.

Consider a perversity $\bar{p}$ on $X$ and a perversity $\bar{q}$ on $Y$ verifying $\bar{p} \geqslant f^{*} \bar{q}$. The induced operator $f^{*}: \widetilde{N}_{\bar{q}}^{*}(Y ; R) \rightarrow \widetilde{N}_{\bar{p}}^{*}(X ; R)$, is a well defined chain map inducing the morphism $f^{*}: \mathscr{H}_{\bar{q}}^{*}(Y ; R) \rightarrow \mathscr{H}_{\bar{p}}^{*}(X ; R)$.

Proof. The definition makes sense since $\left(\delta_{\ell}^{*} f^{*}(\omega)\right)_{\sigma}=\delta_{\ell}^{*}\left(f^{*}(\omega)_{\sigma}\right)=\delta_{\ell}^{*}\left(\omega_{f \circ \sigma}\right)$ $=\omega_{f \circ \sigma \circ \delta_{\ell}}=\left(f^{*}(\omega)\right)_{\sigma \circ \delta_{\ell}}$, for each $\omega \in \tilde{N}^{*}(Y ; R)$, each regular simplex $\sigma: \Delta \rightarrow X$ and each regular face $\delta_{\ell}: \nabla \rightarrow \Delta$.

The induced morphism is a chain map since $\left(\delta f^{*}(\omega)\right)_{\sigma}=\delta\left(f^{*}(\omega)_{\sigma}\right)=\delta\left(\omega_{f \circ \sigma}\right)=$ $(\delta \omega)_{f \circ \sigma}=\left(f^{*}(\delta \omega)\right)_{\sigma}$, for each $\omega \in \tilde{N}^{*}(Y ; R)$ and each regular simplex $\sigma: \Delta_{\sigma} \rightarrow X$.

We turn now to perversities and firstly to the filtrations. The euclidean simplex $\Delta$ has two filtrations, respectively induced by $\sigma$ and $f \circ \sigma$, denoted by $\Delta_{\sigma}$ and $\Delta_{f \circ \sigma}$, following the conventions of Definition 3.1. For each $\ell \in\{0, \ldots, n\}$, the hypothesis $f^{-1}\left(Y_{n-\ell}\right)=X_{n-\ell}$ implies equalities, $\sigma^{-1}\left(X_{n-\ell}\right)=\sigma^{-1} f^{-1}\left(Y_{n-\ell}\right)=$ $(f \circ \sigma)^{-1}\left(Y_{n-\ell}\right)$ and $\Delta_{\sigma}=\Delta_{f \circ \sigma}$.

Let $S$ be an $\ell$-codimensional singular stratum of $X$ such that $S \cap \operatorname{Im} \sigma \neq \varnothing$. The stratum $S^{f}$ of $Y$, characterized by $f(S) \subset S^{f}$, also has codimension $\ell$ and verifies $S^{f} \cap \operatorname{Im}(f \circ \sigma) \neq \varnothing$. Moreover, the definition of $f^{*}$ and the previous observation on the two filtrations of $\Delta$ give $\left\|f^{*}(\omega)_{\sigma}\right\|_{\ell}=\left\|\omega_{f \circ \sigma}\right\|_{\ell}$. From Definition 3.3, we deduce $\left\|f^{*}(\omega)\right\|_{S} \leqslant\|\omega\|_{S^{f}} \leqslant \bar{q}\left(S^{f}\right)$. The assumption made on the perversities $\bar{p}$ and $\bar{q}$ allows us to conclude $\left\|f^{*}(\omega)\right\|_{S} \leqslant \bar{p}(S)$. The same argument applied to the $\bar{q}$-allowable form, $\delta \omega$, gives $\left\|f^{*}(\delta \omega)\right\|_{S} \leqslant \bar{p}(S)$. The compatibility of $f^{*}$ with the differential $\delta$ implies $f^{*}(\omega) \in \widetilde{N}_{\bar{p}}^{*}(X ; R)$.

REMARK 3.6. If $f$ is a stratum preserving stratified map, for any filtered simplex $\sigma: \Delta \rightarrow X$, the $\sigma$ - and $f \circ \sigma$ - decompositions of $\Delta$ are the same: $\Delta_{\sigma}=\Delta_{f \circ \sigma}$. This is not the case for a general stratified map (see [4, A.25]).

\section{Cup product.}

We have defined the notion of cup product in 4 for "filtered face sets". In 8], we also introduced the notion of cup $_{i}$-products and Steenrod squares on the blown-up intersection cohomology. We give below a definition of cup product for any coefficient ring, in our context of stratum depending perversities.

Definition 4.1. Two ordered simplices, $F=\left[a_{0}, \ldots, a_{k}\right]$ and $G=\left[b_{0}, \ldots, b_{\ell}\right]$, of an euclidean simplex $\Delta$, are said to be compatible if $a_{k}=b_{0}$. In this case, we set $F \cup G=\left[a_{0}, \ldots, a_{k}, b_{1}, \ldots, b_{\ell}\right] \in N_{*}(\Delta)$. The cup product is defined on the dual basis of $N^{*}(\Delta)$ by

$$
\mathbf{1}_{F} \cup \mathbf{1}_{G}=(-1)^{k \cdot \ell} \mathbf{1}_{F \cup G}
$$

if $F$ and $G$ are compatible and 0 if not. 
In the case of the cone $\mathrm{c} \Delta$, this law appears on $N^{*}(\mathrm{c} \Delta)$ as:

$$
\mathbf{1}_{(F, \varepsilon)} \cup \mathbf{1}_{(G, \kappa)}=\left\{\begin{array}{cl}
(-1)^{|F| \cdot|(G, \kappa)|} \mathbf{1}_{(F \cup G, \kappa)} & \text { if } F, G \text { compatible and } \varepsilon=0, \\
\mathbf{1}_{(F, \varepsilon)} & \text { if }(G, \kappa)=(\varnothing, 1) \text { and } \varepsilon=1, \\
0 & \text { if not. }
\end{array}\right.
$$

If $\Delta=\Delta_{0} * \cdots * \Delta_{n}$ is a regular euclidean simplex, this definition extend to $\tilde{N}^{*}(\Delta)$ as a law defined on a tensor product. More precisely, if $\omega_{0} \otimes \cdots \otimes \omega_{n}$ and $\eta_{0} \otimes \cdots \otimes \eta_{n}$ belong to $N^{*}\left(\mathrm{c} \Delta_{0}\right) \otimes \cdots \otimes N^{*}\left(\Delta_{n}\right)$, we set

$$
\left(\omega_{0} \otimes \cdots \otimes \omega_{n}\right) \cup\left(\eta_{0} \otimes \cdots \otimes \eta_{n}\right)=(-1)^{\sum_{i>j}\left|\omega_{i}\right|\left|\eta_{j}\right|}\left(\omega_{0} \cup \eta_{0}\right) \otimes \cdots \otimes\left(\omega_{n} \cup \eta_{n}\right) .
$$

Given two perversities $\bar{p}, \bar{q}$ defined on a filtered set $X$ we define the perversity $\bar{p}+\bar{q}$ by $(\bar{p}+\bar{q})(S)=\bar{p}(S)+\bar{q}(S)$, with the convention $-\infty+\bar{p}(S)=\bar{p}(S)-\infty=-\infty$.

Proposition 4.2. For each filtered space $X$ endowed with two perversities $\bar{p}$ and $\bar{q}$, there exists an associative multiplication,

$$
-\cup-: \tilde{N}_{\bar{p}}^{k}(X ; R) \otimes \tilde{N}_{\bar{q}}^{\ell}(X ; R) \rightarrow \tilde{N}_{\bar{p}+\bar{q}}^{k+\ell}(X ; R),
$$

defined by $(\omega \cup \eta)_{\sigma}=\omega_{\sigma} \cup \eta_{\sigma}$, for each pair of cochains $(\omega, \eta) \in \widetilde{N}_{\bar{p}}^{k}(X ; R) \times \tilde{N}_{\bar{q}}^{\ell}(X ; R)$ and each regular simplex $\sigma: \Delta \rightarrow X$. It induces a graded commutative multiplication with unity called intersection cup product,

$$
-\cup-: \mathscr{H}_{\bar{p}}^{k}(X ; R) \otimes \mathscr{H}_{\bar{q}}^{\ell}(X ; R) \rightarrow \mathscr{H}_{\bar{p}+\bar{q}}^{k+\ell}(X ; R) .
$$

PROOF. Begin by verifying that the product locally defined on each regular simplex extends to the blown-up complex. For this, consider two cochains $\omega, \eta \in$ $\tilde{N}^{*}(X ; R)$ and $\delta_{\ell}: \nabla \rightarrow \Delta$ a regular face of a simplex $\sigma: \Delta \rightarrow X$. The map induced by $\delta_{\ell}$ at the cochain level, $\delta_{\ell}^{*}: \tilde{N}^{*}(X ; R) \rightarrow \tilde{N}^{*}(X ; R)$, is the identity on each factor of the tensor product, except for one where it is induced by a canonical inclusion. It is compatible with the cup product and we can write, $\delta_{\ell}^{*}(\omega \cup \eta)_{\sigma}=\delta_{\ell}^{*}\left(\omega_{\sigma} \cup \eta_{\sigma}\right)={ }_{(1)}$ $\delta_{\ell}^{*}\left(\omega_{\sigma}\right) \cup \delta_{\ell}^{*}\left(\eta_{\sigma}\right)=\omega_{\sigma \circ \delta_{\ell}} \cup \eta_{\sigma \circ \delta_{\ell}}=(\omega \cup \eta)_{\sigma \circ \delta_{\ell}}$, where ${ }_{(1)}$ comes from the naturality of the usual cup product. Then the product $\omega \cup \eta$ of the statement is well defined.

Let us now study the behavior of the cup product with respect to the perverse degree. This is a local issue. Let $\omega \in \widetilde{N}_{\bar{p}}^{*}(X ; R)$ and $\eta \in \widetilde{N}_{\bar{q}}^{*}(X ; R)$. We need to prove that

$$
\left\|\omega_{\sigma} \cup \eta_{\sigma}\right\|_{\ell} \leqslant(\bar{p}+\bar{q})(S)
$$

where $S$ is a singular stratum of $X, \ell=\operatorname{codim} S$, and $\sigma: \Delta=\Delta_{0} * \cdots * \Delta_{n} \rightarrow X$ is a regular simplex with $\operatorname{Im} \sigma \cap S \neq \varnothing$.

Without loss of generality, we can suppose $\omega_{\sigma}=\mathbf{1}_{(F, \varepsilon)}$ and $\eta_{\sigma}=\mathbf{1}_{(G, \kappa)}$. The result is clear if $\left\|\omega_{\sigma} \cup \eta_{\sigma}\right\|_{\ell}=-\infty$. So, we can suppose that $\left\|\omega_{\sigma} \cup \eta_{\sigma}\right\|_{\ell} \neq-\infty$. If $\bar{p}(S)=-\infty$, condition $\left\|\omega_{\sigma}\right\|_{\ell} \leqslant-\infty$ implies $\varepsilon_{n-\ell}=1$ and therefore $\left\|\omega_{\sigma} \cup \eta_{\sigma}\right\|_{\ell}=$ $-\infty$. So, we can suppose that $\bar{p}(S)>-\infty$ and similarly $\bar{q}(S)>-\infty$, which imply $(\bar{p}+\bar{q})(S)=\bar{p}(S)+\bar{q}(S)$. Using (4.1) and Definition 2.5. we see that condition $\left\|\omega_{\sigma} \cup \eta_{\sigma}\right\|_{\ell} \neq-\infty$ gives $\varepsilon_{n-\ell}=\kappa_{n-\ell}=0$. The usual degree of the cup product of two cochains gives $\left\|\omega_{\sigma} \cup \eta_{\sigma}\right\|_{\ell} \leqslant\left\|\omega_{\sigma}\right\|_{\ell}+\left\|\eta_{\sigma}\right\|_{\ell} \leqslant \bar{p}(S)+\bar{q}(S)=(\bar{p}+\bar{q})(S)$.

Then, the cup product of a $\bar{p}$-allowable cochain and of a $\bar{q}$-allowable cochain is $(\bar{p}+\bar{q})$-allowable. For the intersection cochains, the result follows from the formula $\delta(\omega \cup \eta)=\delta(\omega) \cup \eta+(-1)^{|\omega|} \omega \cup \delta(\eta)$.

Associativity is deduced from the associativity of the usual cup product. The unity element is the 0 -cochain taking the value 1 on any face. 
Let us verify commutativity. We have constructed the product $-\cup_{1}-: \widetilde{N}_{\bar{p}}^{k}\left(X, \mathbb{Z}_{2}\right) \otimes \widetilde{N}_{\bar{q}}^{\ell}\left(X, \mathbb{Z}_{2}\right) \rightarrow \widetilde{N}_{\bar{p}+\bar{q}}^{k+\ell-1}\left(X, \mathbb{Z}_{2}\right)$ verifying the Leibniz condition $\delta\left(x_{1} \cup_{1} x_{2}\right)=x_{1} \cup x_{2}+x_{2} \cup x_{1}+\delta x_{1} \cup_{1} x_{2}+x_{1} \cup_{1} \delta x_{2}$ (see [8]). For general coefficients, and following the same procedure, we construct the product $-\cup_{1}-: \widetilde{N}_{\bar{p}}^{k}(X ; R) \otimes$ $\tilde{N}_{\bar{q}}^{\ell}(X ; R) \rightarrow \tilde{N}_{\bar{p}+\bar{q}}^{k+\ell-1}(X ; R)$, taking into account the signs (see for example [3, p. 414]). So, for each $\omega \in \tilde{N}_{\bar{p}}^{k}(X ; R)$ and $\eta \in \tilde{N}_{\bar{q}}^{\ell}(X ; R)$ we have

$$
\delta\left(\omega \cup_{1} \eta\right)=(-1)^{p+q-1} \omega \cup \eta+(-1)^{p+q+p q} \eta \cup \omega+\delta \omega \cup_{1} \eta+(-1)^{p} \omega \cup_{1} \delta \eta .
$$

So, if $\delta \omega=\delta \eta=0$, we get the commutativity $[\omega] \cup[\eta]=(-1)^{p q}[\eta] \cup[\omega]$.

See [1, 15] for other cup products.

REMARK 4.3. Given perversities $\bar{a} \leqslant \bar{p}$ and $\bar{b} \leqslant \bar{q}$, the cup product $-\cup$ $-: \tilde{N}_{\bar{a}}^{*}(X ; R) \otimes \tilde{N}_{\bar{b}}^{*}(X ; R) \rightarrow \tilde{N}_{\bar{a}+\bar{b}}^{*}(X ; R)$ is the restriction of $-\cup-: \tilde{N}_{\bar{p}}^{*}(X ; R) \otimes$ $\tilde{N}_{\bar{q}}^{*}(X ; R) \rightarrow \tilde{N}_{\bar{p}+\bar{q}}^{*}(X ; R)$.

\section{Intersection and tame intersection (co)homology.}

For a perversity $\bar{p}$ such that $\bar{p} \nless \bar{t}$, we may have $\bar{p}$-allowable simplices that are not regular. This failure has bad consequences; for instance, Poincaré duality may be not satisfied in this case (see [6]). To overcome this problem the tame intersection homology $\mathfrak{H}_{*}^{\bar{T}}(X ; R)$ has been introduced in [24]. In this work, we use the simpler presentation of G. Friedman (see [9, 14, 5]).

We begin by recalling the notions of intersection homology. As proved in 4 , Proposition A29], it can be computed using filtered chains.

Definition 5.1. Consider a perverse space $(X, \bar{p})$ and a filtered $\operatorname{simplex} \sigma: \Delta=$ $\Delta_{0} * \cdots * \Delta_{n} \rightarrow X$.

(i) The perverse degree of $\sigma$ is the $(n+1)$-tuple, $\|\sigma\|=\left(\|\sigma\|_{0}, \ldots,\|\sigma\|_{n}\right)$, where $\|\sigma\|_{i}=\operatorname{dim} \sigma^{-1}\left(X_{n-i}\right)=\operatorname{dim}\left(\Delta_{0} * \cdots * \Delta_{n-i}\right)$, with the convention $\operatorname{dim} \varnothing=$ $-\infty$.

(ii) Given a stratum $S$ of $X$, the perverse degree of $\sigma$ along $S$ is defined by

$$
\|\sigma\|_{S}=\left\{\begin{array}{cc}
-\infty, & \text { if } S \cap \operatorname{Im} \sigma=\varnothing, \\
\|\sigma\|_{\operatorname{codim} S} & \text { if } S \cap \operatorname{Im} \sigma \neq \varnothing .
\end{array}\right.
$$

(iii) The filtered singular simplex $\sigma$ is $\bar{p}$-allowable if $\|\sigma\|_{S} \leqslant \operatorname{dim} \Delta-\operatorname{codim} S+\bar{p}(S)$, for any stratum $S$.

(iv) A chain $c$ is $\bar{p}$-allowable if it is a linear combination of $\bar{p}$-allowable simplices. The chain $c$ is a $\bar{p}$-intersection chain if $c$ and $\partial c$ are $\bar{p}$-allowable chains.

Definition 5.2. Consider a perverse space $(X, \bar{p})$. We denote by $C_{*}(X ; R)$ the complex of filtered chains of $X$, generated by filtered simplices. The dual complex is $C^{*}(X ; R)=\operatorname{hom}\left(C_{*}(X ; R), R\right)$.

The complex of $\bar{p}$-intersection chains of $X$ with the differential $\partial$ is denoted by $C_{*}^{\bar{p}}(X ; R)$. Its homology $H_{*}^{\bar{p}}(X ; R)$ is the $\bar{p}$-intersection homology of $X$ (see [5. Théorème A]). The dual complex $C_{\bar{p}}^{*}(X ; R)=\operatorname{hom}\left(C_{*}^{\bar{p}}(X ; R), R\right)$ computes the $\bar{p}$-intersection cohomology $H_{\bar{p}}^{*}(X ; R)$ (see [14]). 
Definition 5.3. Given a regular simplex $\Delta=\Delta_{0} * \cdots * \Delta_{n}$ we denote by $\mathfrak{d}$ the regular part of the chain $\partial \Delta$. That is $\mathfrak{d} \Delta=\partial\left(\Delta_{0} * \cdots * \Delta_{n-1}\right) * \Delta_{n}$, if $\left|\Delta_{n}\right|=0$, or $\mathfrak{d} \Delta=\partial \Delta$, if $\left|\Delta_{n}\right| \geqslant 1$.

Definition 5.4. Let $(X, \bar{p})$ be a perverse space. Given a regular simplex $\sigma: \Delta \rightarrow X$, we define the chain $\mathfrak{d} \sigma$ by $\sigma \circ \mathfrak{d}$. Notice that $\mathfrak{d}^{2}=0$. We denote by $\mathfrak{C}_{*}(X ; R)$ the chain complex generated by the regular simplices, endowed with the differential $\mathfrak{d}$.

Definition 5.5. Let $(X, \bar{p})$ be a perverse space. A $\bar{p}$-allowable filtered simplex $\sigma: \Delta \rightarrow X$ is $\bar{p}$-tame if $\sigma$ is also a regular simplex. A chain $\xi$ is $\bar{p}$-tame if is a linear combination of $\bar{p}$-tame simplices. The chain $\xi$ is a tame $\bar{p}$-intersection chain if $\xi$ and $\mathfrak{d} \xi$ are $\bar{p}$-tame chains.

We write $\mathfrak{C}_{*}^{\bar{p}}(X ; R) \subset \mathfrak{C}_{*}(X ; R)$ the complex of tame $\bar{p}$-intersection chains endowed with the differential $\mathfrak{d}$. Its homology $\mathfrak{H}_{*}^{\bar{p}}(X ; R)$ is the tame $\bar{p}$-intersection homology of $X$

Main properties of this homology have been studied in $[\mathbf{5}, \mathbf{1 4}$. We have proven in [5] that the homology $\mathfrak{H}_{*}^{\bar{p}}(X ; R)$ coincides with those of [24, 10] (see also [14, Chapter 6]). It is also proved that $\mathfrak{H}_{*}^{\bar{p}}(X ; R)=H_{*}^{\bar{p}}(X ; R)$ if $\bar{p} \leqslant \bar{t}$. The tame $\bar{p}$ intersection cohomology $\mathfrak{H}_{\bar{p}}^{*}(X ; R)$ is defined by using the dual complex $\mathfrak{C}_{\bar{p}}^{*}(X ; R)=$ $\operatorname{Hom}\left(\mathfrak{C}_{*}^{\bar{p}}(X ; R), R\right)$. It verifies $\mathfrak{H}_{\bar{p}}^{*}(X ; R)=H_{\bar{p}}^{*}(X ; R)$ if $\bar{p} \leqslant \bar{t}$.

5.1. Shifted filtrations. Intersection homology does not depend on the dimension of the strata of $X$ but on the codimension of these strata (see 14, Section 4.3.1]). Let us consider on $X$ the shifted filtration $Y$ of (3.2) in subsection 3.1. So, the formal dimension of $Y$ is $n+m$. Following subsection 3.1 we have:

$$
\|\sigma\|_{\ell}^{X}=\operatorname{dim} \sigma^{-1}\left(X_{n-\ell}\right)=\operatorname{dim} \sigma^{-1}\left(Y_{n+m-\ell}\right)=\|\sigma\|_{\ell}^{Y} .
$$

The allowability condition is the same. The perversity $\bar{p}$ is also a perversity on $Y$. If $S$ is a stratum of $X$ (and therefore of $Y$ ) with $\operatorname{Im} \sigma \cap S \neq \varnothing$ we have $\ell=\operatorname{codim}_{X} S=\operatorname{codim}_{Y} S$. So,

$$
\|\sigma\|_{\ell}^{X} \leqslant \operatorname{dim} \Delta-\ell+\bar{p}(S) \Longleftrightarrow\|\sigma\|_{\ell}^{Y} \leqslant \operatorname{dim} \Delta-\ell+\bar{p}(S) .
$$

We get $C_{*}^{\bar{p}}(X ; R)=C_{*}^{\bar{p}}(Y ; R)$ and therefore $H_{*}^{\bar{p}}(X ; R)=H_{*}^{\bar{p}}(Y ; R)$. By the same reasons, we also have $H_{\bar{p}}^{*}(X ; R)=H_{\bar{p}}^{*}(Y ; R), \mathfrak{H}_{*}^{\bar{p}}(X ; R)=\mathfrak{H}_{*}^{\bar{p}}(Y ; R)$ and $\mathfrak{H}_{\bar{p}}^{*}(X ; R)=\mathfrak{H}_{\bar{p}}^{*}(Y ; R)$.

\section{Cap product.}

We introduce the notion of cap product for the blown-up intersection (co)homology, already treated in [7] in a different context. First of all, we work at the simplex level.

Let $\Delta$ be an $m$-dimensional euclidean simplex. We denote by $[\Delta]$ its face of maximal dimension. The classical cap product $-\cap[\Delta]: N^{*}(\Delta) \rightarrow N_{m-*}(\Delta)$ is defined by

(6.1) $\mathbf{1}_{F} \cap[\Delta]= \begin{cases}G & \text { if } F \cup G=\Delta \text { (where } \cup \text { is the map of Definition 4.1), } \\ 0 & \text { otherwise. }\end{cases}$ 
Consider now the cone $\mathrm{c} \Delta$ whose apex is denoted by $\mathrm{v}$, which is the last vertex of the cone (see page 7). We have the formula:

$$
\mathbf{1}_{(F, j)} \cap[\mathrm{c} \Delta]=\left\{\begin{array}{cl}
(G, 1) & \text { if } j=0 \text { or }(F, j)=(\Delta, 1), \\
0 & \text { if not, }
\end{array}\right.
$$

where the simplex $G$ is the face of $\Delta$ with $F \cup G=\Delta$ (cf. Definition 4.1) if $j=0$ and $G=\varnothing$ if $(F, j)=(\Delta, 1)$.

We extend it to regular simplices $\Delta=\Delta_{0} * \cdots * \Delta_{n}$ as follows. Denote $\tilde{N}_{*}(\Delta)=$ $N_{*}\left(\mathrm{c} \Delta_{0}\right) \otimes \cdots \otimes N_{*}\left(\mathrm{c} \Delta_{n-1}\right) \otimes N_{*}\left(\Delta_{n}\right)$.

Definition 6.1. We define the cap product $-\widetilde{\cap} \widetilde{\Delta}: \widetilde{N}^{*}(\Delta) \rightarrow \widetilde{N}_{*-m}(\Delta)$, linearly from

$$
\mathbf{1}_{(F, \varepsilon)} \widetilde{\cap} \widetilde{\Delta}=(-1)^{\nu(F, \varepsilon, \Delta)}\left(\mathbf{1}_{\left(F_{0}, \varepsilon_{0}\right)} \cap \mathrm{c}\left[\Delta_{0}\right]\right) \otimes \cdots \otimes\left(\mathbf{1}_{F_{n}} \cap\left[\Delta_{n}\right]\right),
$$

where $\mathbf{1}_{(F, \varepsilon)}=\mathbf{1}_{\left(F_{0}, \varepsilon_{0}\right)} \otimes \cdots \otimes \mathbf{1}_{\left(F_{n-1}, \varepsilon_{n-1}\right)} \otimes \mathbf{1}_{F_{n}}, \widetilde{\Delta}=\mathrm{c} \Delta_{0} \times \cdots \times \mathrm{c} \Delta_{n-1} \times \Delta_{n}$ (see (2.3) ) and $\nu(F, \varepsilon, \Delta)=\sum_{j=0}^{n-1}\left(\operatorname{dim} \Delta_{j}+1\right)\left(\sum_{i=j+1}^{n}\left|\left(F_{i}, \varepsilon_{i}\right)\right|\right)$, with the convention $\varepsilon_{n}=0$.

At the filtered simplex level, the local intersection cap product $-\cap \widetilde{\Delta}: \widetilde{N}^{*}(\Delta) \rightarrow$ $N_{m-*}(\Delta)$ is defined by

$$
c \cap \widetilde{\Delta}=\mu_{*}(c \widetilde{\cap} \widetilde{\Delta}),
$$

where the map $\mu_{*}: \tilde{N}_{*}(\Delta) \rightarrow N_{*}(\Delta)$ is defined by

$$
\mu_{*}\left(\otimes_{k=0}^{n-1}\left(F_{k}, \varepsilon_{k}\right) \otimes F_{n}\right)=\left\{\begin{array}{cl}
F_{0} * \cdots * F_{\ell} & \text { if } \operatorname{dim}(F, \varepsilon)=\operatorname{dim}\left(F_{0} * \cdots * F_{\ell}\right), \\
0 & \text { otherwise, }
\end{array}\right.
$$

where $(F, \varepsilon)=\left(F_{0}, \varepsilon_{0}\right) \otimes \cdots \otimes\left(F_{n-1}, \varepsilon_{n-1}\right) \otimes F_{n}$ and $\ell$ is the smallest integer, $j$, such that $\varepsilon_{j}=0$.

Definition 6.2. Since $\widetilde{\Delta}=\mathrm{c} \Delta_{0} \times \cdots \times \mathrm{c} \Delta_{n-1} \times \Delta_{n}$ (see (2.3) $)$, we have the boundary chain

$$
\begin{aligned}
\partial \widetilde{\Delta}= & \sum_{\substack{i=0 \\
\Delta_{i} \neq \varnothing}}^{n}(-1)^{|\Delta|_{\leqslant i-1+1}}\left[\mathrm{c} \Delta_{0}\right] \otimes \cdots \otimes\left[\mathrm{c} \partial \Delta_{i}\right] \otimes \cdots \otimes\left[\Delta_{n}\right] \\
& +\sum_{\substack{i=0 \\
\Delta_{i} \neq \varnothing}}^{n-1}(-1)^{|\Delta|_{\leqslant i+1}}\left[\mathrm{c} \Delta_{0}\right] \otimes \cdots \otimes\left[\Delta_{i}\right] \otimes \cdots \otimes\left[\Delta_{n}\right] .
\end{aligned}
$$

Let $i \in\{0, \ldots, n-1\}$ such that $\Delta_{i} \neq \varnothing$, the face $\mathcal{H}_{i}=\mathrm{c} \Delta_{0} \times \cdots \times \mathrm{c} \Delta_{i-1} \times \Delta_{i} \times$ $\mathrm{c} \Delta_{i+1} \times \cdots \times \Delta_{n}$ is called a hidden face of $\widetilde{\Delta}$. This gives

$$
\partial \widetilde{\Delta}=\widetilde{\mathfrak{d} \Delta}+\sum_{\substack{i=0 \\ \Delta_{i} \neq \varnothing}}^{n-1}(-1)^{|\Delta|_{\leqslant i+1}} \mathcal{H}_{i},
$$

where $\widetilde{d} \Delta$ is the blow up of the weighted simplicial complex $\mathfrak{d} \Delta$ (cf. Definition 2.2).

REMARK 6.3. 
- For each $\omega \in \tilde{N}^{*}(\Delta)$ the chain $\omega \cap \widetilde{\Delta}$ is regular or 0 . Let us see that. Let $\mathbf{1}_{(F, \varepsilon)}=\mathbf{1}_{\left(F_{0}, \varepsilon_{0}\right)} \otimes \cdots \otimes \mathbf{1}_{F_{n}} \in \tilde{N}^{*}(\Delta)$. From the definition of the cap product we have, up to a sign,

$$
\mathbf{1}_{(F, \varepsilon)} \cap \widetilde{\Delta}=G_{0} * \cdots * G_{n}
$$

if $\mathbf{1}_{(F, \varepsilon)} \cap \widetilde{\Delta} \neq 0$. The faces $G_{\bullet}$ have been defined in (6.1), (6.2) and (6.4).

The simplex $\mathbf{1}_{(F, \varepsilon)} \cap \widetilde{\Delta}$ is regular: $F_{n} \neq \varnothing \Rightarrow G_{n} \neq \varnothing$.

- Given a hidden face $\mathcal{H}_{i}=\mathrm{c} \Delta_{0} \times \cdots \times \Delta_{i} \times \cdots \times \Delta_{n}$, we have

$$
\mu_{*}\left(\mathcal{H}_{i}\right)=\left\{\begin{array}{cl}
\Delta_{0} * \cdots * \Delta_{i} & \text { if } \operatorname{dim}\left(\Delta_{i+1} * \cdots * \Delta_{n}\right)=0 \\
0 & \text { otherwise }
\end{array}\right.
$$

which is a non regular face or 0 .

We define the intersection cap product for any filtered space $X$.

Definition 6.4. Let $X$ be a filtered space. The intersection cap product

$$
-\cap-: \tilde{N}^{*}(X ; R) \otimes \mathfrak{C}_{m}(X ; R) \rightarrow \mathfrak{C}_{m-*}(X ; R)
$$

is defined by linear extension of

$$
\omega \cap \sigma=\sigma_{*}\left(\omega_{\sigma} \cap \widetilde{\Delta}\right)
$$

where $\omega \in \tilde{N}^{*}(X ; R)$ and $\sigma: \Delta \rightarrow X$ is a regular simplex.

Lemma 6.5. Let $\Delta$ be a regular simplex. The map $\mu_{*}: \tilde{N}_{*}(\Delta) \rightarrow N_{*}(\Delta)$ commutes with differentials.

Proof. Set $\Delta=\Delta_{0} * \cdots * \Delta_{n}$. We proceed by induction on $n$. The result is clear when $n=0$. We consider the regular simplex $\nabla=\Delta_{1} * \cdots * \Delta_{n}$, et $\widetilde{N}_{*}(\nabla)=N_{*}\left(\mathrm{c} \Delta_{1}\right) \otimes \cdots \otimes N_{*}\left(\mathrm{c} \Delta_{n-1}\right) \otimes N_{*}\left(\Delta_{n}\right)$. The map $\mu_{\Delta, *}$ is the composition

$$
\mu_{\Delta, *}: N_{*}\left(\mathrm{c} \Delta_{0}\right) \otimes \tilde{N}_{*}(\nabla) \stackrel{\operatorname{id} \otimes \mu_{\nabla, *}}{\longrightarrow} N_{*}\left(\mathrm{c} \Delta_{0}\right) \otimes N_{*}(\nabla) \stackrel{\nu}{\rightarrow} N_{*}(\Delta),
$$

(see (6.4) $)$, where

$$
\nu\left(\left(F_{0}, \varepsilon_{0}\right) \otimes G\right)=\left\{\begin{array}{cl}
F_{0} & \text { if } \varepsilon_{0}=0 \text { and } \operatorname{dim} G=0, \\
0 & \text { if } \varepsilon_{0}=0 \text { and } \operatorname{dim} G>0, \\
F_{0} * G & \text { if } \varepsilon_{0}=1 .
\end{array}\right.
$$

By induction hypothesis, the operator $\mu_{\nabla, *}$ is compatible with differentials. It remains to prove that $\nu$ is also compatible with differentials. We distinguish four cases.

- If $\varepsilon_{0}=0$ and $\operatorname{dim} G=0$, we have $\nu\left(\partial\left(\left(F_{0}, 0\right) \otimes G\right)\right)=\nu\left(\left(\partial F_{0}, 0\right) \otimes G\right)=$ $\partial F_{0}=\partial \nu\left(\left(F_{0}, 0\right) \otimes G\right)$.

- If $\varepsilon_{0}=0$ and $\operatorname{dim} G=1$, we have $\nu\left(\partial\left(\left(F_{0}, 0\right) \otimes G\right)\right)=\nu\left(\left(\partial F_{0}, 0\right) \otimes G\right)+$ $(-1)^{\left|F_{0}\right|} \nu\left(\left(F_{0}, 0\right) \otimes \partial G\right)=0+F_{0}-F_{0}=0=\partial \nu\left(\left(F_{0}, 0\right) \otimes G\right)$.

- If $\varepsilon_{0}=0$ and $\operatorname{dim} G>1$, all the terms vanish.

- If $\varepsilon_{0}=1$, the result comes from

$$
\begin{array}{r}
\nu\left(\partial\left(\left(F_{0}, 1\right) \otimes G\right)\right)= \\
\nu\left(\left(\partial F_{0}, 1\right) \otimes G\right)+(-1)^{\left|F_{0}\right|+1}\left(\nu\left(\left(F_{0}, 0\right) \otimes G\right)+\nu\left(\left(F_{0}, 1\right) \otimes \partial G\right)\right)= \\
\left(\partial F_{0}\right) * G+(-1)^{\left|F_{0}\right|+1}\left\{\begin{array}{cc}
F_{0} & \text { if } \operatorname{dim} G=0, \\
F_{0} * \partial G & \text { if } \operatorname{dim} G \geqslant 1, \\
\partial\left(F_{0} * G\right)=\partial \nu\left(\left(F_{0}, 1\right) \otimes G\right) .
\end{array}\right.
\end{array}
$$


Proposition 6.6. The intersection cap product verifies the following properties

(i) $\mathfrak{d}(\omega \cap \xi)=(\delta \omega) \cap \xi+(-1)^{|\omega|} \omega \cap \mathfrak{d} \xi$, and

(ii) $(\omega \cup \eta) \cap \xi=(-1)^{|\omega| \cdot|\eta|} \eta \cap \omega \cap \xi$.

where $\omega, \eta \in \tilde{N}^{*}(X ; R)$ and $\xi \in \mathfrak{C}_{*}(X ; R)$.

Proof. (i) It suffices to prove it for a regular simplex $\sigma: \Delta \rightarrow X$. We write $k=|\omega|$. Using formula (6.5), the classic Leibniz formula for the cap product gives

$$
\begin{aligned}
& \partial\left(\omega_{\sigma} \cap \widetilde{\Delta}\right)=\partial \mu_{*}\left(\omega_{\sigma} \widetilde{\cap} \widetilde{\Delta}\right) \stackrel{\text { Lemma } 6.5}{=} \mu_{*} \partial\left(\omega_{\sigma} \widetilde{\cap} \widetilde{\Delta}\right)= \\
& \mu_{*}\left(\left(\delta \omega_{\sigma}\right) \tilde{\cap} \widetilde{\Delta}+(-1)^{k} \omega_{\sigma} \tilde{\cap}(\partial \widetilde{\Delta})\right)= \\
& \stackrel{\text { 6.5. }}{=} \mu_{*}\left(\left(\delta \omega_{\sigma}\right) \widetilde{\cap} \widetilde{\Delta}+(-1)^{k} \omega_{\sigma} \widetilde{\cap} \widetilde{\mathfrak{d} \Delta}+\sum_{\substack{i=0 \\
\Delta_{i} \neq \varnothing}}^{n-1}(-1)^{(-1)^{k+|\Delta| \leqslant i}} \omega_{\sigma} \widetilde{\cap} \mathcal{H}_{i}\right)= \\
& =\underbrace{\mu_{*}\left(\left(\delta \omega_{\sigma}\right) \widetilde{\cap} \widetilde{\Delta}+(-1)^{k} \omega_{\sigma} \widetilde{\cap} \widetilde{\mathfrak{d} \Delta}\right)}_{\text {regular or } 0}+\sum_{\substack{i=0 \\
\Delta_{i} \neq \varnothing}}^{n-1}(-1)^{(-1)^{k+|\Delta|_{\leqslant i}}} \underbrace{\mu_{*}\left(\omega_{\sigma} \widetilde{\cap} \mathcal{H}_{i}\right)}_{\text {non regular or } 0}
\end{aligned}
$$

(cf. Remark 6.3). So, $\mathfrak{d}\left(\omega_{\sigma} \cap \widetilde{\Delta}\right)=\mu_{*}\left(\left(\delta \omega_{\sigma}\right) \widetilde{\cap} \widetilde{\Delta}\right)+(-1)^{k} \mu_{*}\left(\omega_{\sigma} \widetilde{\cap} \widetilde{\mathfrak{d} \Delta}\right)=\left(\delta \omega_{\sigma}\right) \cap$ $\widetilde{\Delta}+(-1)^{k} \omega_{\sigma} \cap \widetilde{d} \Delta$, and therefore $\sigma_{*} \mathfrak{d}\left(\omega_{\sigma} \cap \widetilde{\Delta}\right)=(\delta \omega) \cap \Delta+(-1)^{k} \omega \cap \mathfrak{d} \Delta$.

The regular simplex $\sigma: \Delta \rightarrow X$ is in fact a stratified map when one considers on $\Delta$ the filtration $\Delta_{0} \subset \Delta_{0} * \Delta_{1} \subset \cdots \subset \Delta_{0} * \cdots * \Delta_{n}$. So, $\sigma_{*} \circ \partial=\partial \circ \sigma_{*}$, 4 , Theorem F]. Since $\Delta_{0} * \cdots * \Delta_{n-1}=\sigma^{-1}\left(\Sigma_{X}\right)$ then $\sigma_{*} \circ \mathfrak{d}=\mathfrak{d} \circ \sigma_{*}$. We get property (i) since $\mathfrak{d}(\omega \cap \Delta)=\mathfrak{d} \sigma_{*}\left(\omega_{\sigma} \cap \widetilde{\Delta}\right)=\sigma_{*} \mathfrak{d}\left(\omega_{\sigma} \cap \widetilde{\Delta}\right)$.

(ii) Without loss of generality we can suppose $\omega_{\sigma}=\mathbf{1}_{(F, \varepsilon)}=\mathbf{1}_{\left(F_{0}, \varepsilon_{0}\right)} \otimes \cdots \otimes$ $\mathbf{1}_{\left(F_{n-1}, \varepsilon_{n-1}\right)} \otimes \mathbf{1}_{F_{n}}$ and $\eta_{\sigma}=\mathbf{1}_{(H, \kappa)}=\mathbf{1}_{\left(H_{0}, \kappa_{0}\right)} \otimes \cdots \otimes \mathbf{1}_{\left(H_{n-1}, \kappa_{n-1}\right)} \otimes \mathbf{1}_{H_{n}}$. It suffices to prove

$$
\left(\mathbf{1}_{(F, \varepsilon)} \cup \mathbf{1}_{(H, \kappa)}\right) \widetilde{\cap} \widetilde{\Delta}=(-1)^{|(F, \varepsilon)| \cdot|(H, \kappa)|} \mathbf{1}_{(H, \kappa)} \widetilde{\cap}\left(\mathbf{1}_{(F, \varepsilon)} \widetilde{\cap} \widetilde{\Delta}\right) .
$$


For $n=0$ we find the classic property of cup/cap products. In the general case, with the convention $\varepsilon_{n}=\kappa_{n}=0$, we have

$$
\begin{aligned}
& \left(\mathbf{1}_{(F, \varepsilon)} \cup \mathbf{1}_{(H, \kappa)}\right) \widetilde{\cap} \widetilde{\Delta}=\left(\bigotimes_{a=0}^{n} \mathbf{1}_{\left(F_{a}, \varepsilon_{a}\right)} \cup \bigotimes_{a=0}^{n} \mathbf{1}_{\left(H_{a}, \kappa_{a}\right)}\right) \widetilde{\sim} \widetilde{\Delta} \stackrel{(4.2)}{=} \\
& (-1)^{\ltimes_{1}} \cdot\left(\bigotimes_{a=0}^{n}\left(\mathbf{1}_{\left(F_{a}, \varepsilon_{a}\right)} \cup \mathbf{1}_{\left(H_{a}, \kappa_{a}\right)}\right)\right) \widetilde{\cap} \widetilde{\Delta} \stackrel{(6.3)}{=} \\
& (-1)^{\ltimes_{2}} \cdot \bigotimes_{a=0}^{n-1}\left(\left(\mathbf{1}_{\left(F_{a}, \varepsilon_{a}\right)} \cup \mathbf{1}_{\left(H_{a}, \kappa_{a}\right)}\right) \cap\left[c \Delta_{a}\right]\right) \otimes\left(\left(\mathbf{1}_{F_{n}} \cup \mathbf{1}_{H_{n}}\right) \cap\left[\Delta_{n}\right]\right) \stackrel{\text { classic }}{=} \\
& (-1)^{\ltimes_{3}} \cdot \bigotimes_{a=0}^{n-1}\left(\mathbf{1}_{\left(H_{a}, \kappa_{a}\right)} \cap \mathbf{1}_{\left(F_{a}, \varepsilon_{a}\right)} \cap\left[\mathrm{c} \Delta_{a}\right]\right) \otimes\left(\mathbf{1}_{H_{n}} \cap \mathbf{1}_{F_{n}} \cap\left[\Delta_{n}\right]\right) \stackrel{(6.3)}{=} \\
& (-1)^{\ltimes_{4}} \cdot \mathbf{1}_{(H, \kappa)} \widetilde{\cap}\left(\bigotimes_{a=0}^{n-1}\left(\mathbf{1}_{\left(F_{a}, \varepsilon_{a}\right)} \cap\left[\mathrm{c} \Delta_{a}\right]\right) \otimes\left(\mathbf{1}_{F_{n}} \cap\left[\Delta_{n}\right]\right)\right) \stackrel{(6.3)}{=} \\
& (-1)^{|(F, \varepsilon)| \cdot|(H, \kappa)|} \cdot \mathbf{1}_{(H, \kappa)} \widetilde{\cap} \mathbf{1}_{(F, \varepsilon)} \widetilde{\cap} \widetilde{\Delta} .
\end{aligned}
$$

where $\ltimes_{1}=\sum_{i>j}\left|\left(F_{i}, \varepsilon_{i}\right)\right| \cdot\left|\left(H_{j}, \kappa_{j}\right)\right|, \ltimes_{2}=\ltimes_{1}+\sum_{j=0}^{n-1}\left(\operatorname{dim} \Delta_{j}+1\right)\left(\sum_{i>j}\left|\left(F_{i}, \varepsilon_{i}\right)\right|+\right.$ $\left.\left|\left(H_{i}, \kappa_{i}\right)\right|\right), \ltimes_{3}=\ltimes_{2}+\sum_{i}\left|\left(F_{i}, \varepsilon_{i}\right)\right| \cdot\left|\left(H_{i}, \kappa_{i}\right)\right|$ and

$$
\begin{aligned}
\ltimes_{4}= & \ltimes_{3}+\sum_{j=0}^{n-1}\left(\operatorname{dim} \Delta_{j}+1-\left|\left(F_{j}, \varepsilon_{j}\right)\right|\right)\left(\sum_{i>j}\left|\left(H_{i}, \kappa_{i}\right)\right|\right)=\sum_{i \geqslant j}\left|\left(F_{i}, \varepsilon_{i}\right)\right| \cdot\left|\left(H_{j}, \kappa_{j}\right)\right| \\
& +\sum_{j=0}^{n-1}\left(\operatorname{dim} \Delta_{j}+1\right)\left(\sum_{i>j}\left|\left(F_{i}, \varepsilon_{i}\right)\right|\right)-\sum_{j=0}^{n-1}\left|\left(F_{j}, \varepsilon_{j}\right)\right|\left(\sum_{i>j}\left|\left(H_{i}, \kappa_{i}\right)\right|\right) \\
= & \sum_{j=0}^{n-1}\left(\operatorname{dim} \Delta_{j}+1\right)\left(\sum_{i>j}\left|\left(F_{i}, \varepsilon_{i}\right)\right|\right)+|(F, \varepsilon)| \cdot|(H, \kappa)| .
\end{aligned}
$$

The cap product between a cochain $\omega \in \tilde{N}^{*}(X ; R)$ and a chain $\xi \in C^{*}(X ; R)$ may not exist. This happens when a simplex $\sigma$ of $\xi$ lies in the singular part of $X$. In this case, $\sigma$ is not regular and we cannot construct the blow up of $\Delta_{\sigma}$. This is the reason to use the tame intersection homology in the definition of the cap product: all the involved simplices are regular.

Proposition 6.7. For each filtered space, $X$, endowed with two perversities, $\bar{p}$ and $\bar{q}$, the intersection cap product

$$
-\cap-: \tilde{N}_{\bar{p}}^{*}(X ; R) \otimes \mathfrak{C}_{m}^{\bar{q}}(X ; R) \rightarrow \mathfrak{C}_{m-*}^{\bar{p}+\bar{q}}(X ; R),
$$

is well defined and induces a morphism

$$
-\cap-: \mathscr{H}_{\bar{p}}^{*}(X ; R) \otimes \mathfrak{H}_{m}^{\bar{q}}(X ; R) \rightarrow \mathfrak{H}_{m-*}^{\bar{p}+\bar{q}}(X ; R) .
$$

Proof. Following Proposition 6.6 it remains to study the behavior of the cap product with respect to the perverse degree on a singular stratum $S$. Let us consider $\omega \in \tilde{N}^{*}(X ; R)$ with $\|\omega\|_{S} \leqslant \bar{p}(S), \sigma: \Delta \rightarrow X$ a regular simplex with $\operatorname{Im} \sigma \cap S \neq \varnothing$ and $\|\sigma\|_{S} \leqslant|\Delta|-\ell+\bar{q}(S)$, where $\ell=\operatorname{codim} S$. We need to prove

$$
\|\omega \cap \sigma\|_{S} \leqslant|\omega \cap \sigma|-\ell+(\bar{p}+\bar{q})(S)
$$


Without loss of generality, we can suppose $\omega \cap \sigma \neq 0$.

When $\bar{p}(S)=-\infty$, the condition $\|\omega\|_{S} \leqslant-\infty$ implies $\varepsilon_{n-\ell}=1$ and therefore the $(n-\ell)$-factor of $\omega_{\sigma} \widetilde{\cap} \widetilde{\Delta}$ is $\left[\mathrm{v}_{n-\ell}\right]$ which gives $\omega \cap \sigma \cap S=\varnothing$ and therefore (6.6). On the other hand, if $\bar{q}(S)=-\infty$, the condition $\|\sigma\|_{S}=-\infty$ gives $\Delta_{n-\ell}=\varnothing$ then $\omega \cap \sigma \cap S=\varnothing$ and therefore (6.6).

We can suppose $(\bar{p}+\bar{q})(S)=\bar{p}(S)+\bar{q}(S)$. Then we have (6.6) from

$\|\omega \cap \sigma\|_{S} \stackrel{\operatorname{claim}}{\leqslant}\|\sigma\|_{S}+\left\|\omega_{\sigma}\right\|_{\ell}-\left|\omega_{\sigma}\right| \leqslant|\Delta|-\ell+\bar{p}(S)+\bar{q}(S)-\left|\omega_{\sigma}\right|=|\omega \cap \sigma|-\ell+\bar{p}(S)+\bar{q}(S)$

We verify the claim. It suffices to consider $\omega_{\sigma}=\mathbf{1}_{(F, \varepsilon)}$ with $\mathbf{1}_{(F, \varepsilon)} \cap \widetilde{\Delta} \neq 0$. We know, from Remark 6.3, that $\mathbf{1}_{(F, \varepsilon)} \cap \Delta= \pm G_{0} * \cdots * G_{n}$ with $\left|\Delta_{i}\right|=\left|G_{i}\right|+\left|\left(F_{i}, \varepsilon_{i}\right)\right|$, for each $i \in\{0, \ldots, n\}$. Then, $\|\omega \cap \sigma\|_{S}$ is equal to

$$
\begin{aligned}
\left|G_{0} * \cdots * G_{n-\ell}\right| & =\left|G_{0}\right|+\cdots+\left|G_{n-\ell}\right|+n-\ell \\
& =\left|\Delta_{0}\right|+\cdots+\left|\Delta_{n-\ell}\right|+n-\ell-\left|\left(F_{0}, \varepsilon_{0}\right)\right|-\cdots-\left|\left(F_{n-\ell}, \varepsilon_{n-\ell}\right)\right| \\
& =\|\sigma\|_{S}-|(F, \varepsilon)|+|(F, \varepsilon)|_{>n-\ell}=\|\sigma\|_{S}-\left|\omega_{\sigma}\right|+\left\|\omega_{\sigma}\right\|_{\ell} .
\end{aligned}
$$

REMARK 6.8. The intersection cap product is natural respectively to perversities. Given perversities $\bar{a} \leqslant \bar{p}$ and $\bar{b} \leqslant \bar{q}$, the cap product $-\cap-: \widetilde{N}_{\bar{a}}^{*}(X) \otimes \mathfrak{C}_{m}^{\bar{b}}(X) \rightarrow$ $\mathfrak{C}_{m-*}^{\bar{a}+\bar{b}}(X)$ is the restriction of $-\cap-: \widetilde{N}_{\bar{p}}^{*}(X) \otimes \mathfrak{C}_{m}^{\bar{q}}(X) \rightarrow \mathfrak{C}_{m-*}^{\bar{p}+\bar{q}}(X)$.

\section{Stratified maps: the local level.}

In this section and the next one, we prove that any stratified map induces a homomorphism between the blown-up intersection cohomologies if a certain compatibility of the involved perversities is satisfied. In particular, if $\bar{p}$ is a GM-perversity, any stratified map $f: X \rightarrow Y$ induces a homomorphism $f: \mathscr{H}_{\bar{p}}^{*}(X ; R) \rightarrow \mathscr{H}_{\bar{p}}^{*}(Y ; R)$.

Let $f: X \rightarrow Y$ be a stratified map as in Definition [1.5. If $\sigma: \Delta \rightarrow X$ is a filtered simplex then the composite $f \circ \sigma: \Delta \rightarrow Y$ is also a filtered simplex but, in general, the two filtrations induced on $\Delta$ by $\sigma$ and $f \circ \sigma$ differ. We denote them by $\Delta_{\sigma}$ and $\Delta_{f \circ \sigma}$. In [4, Corollary A.25 and Lemma A.24], we prove that the two filtrations $\Delta_{\sigma}$ and $\Delta_{f \circ \sigma}$ (on the same euclidean simplex) match through a finite number of elementary amalgamations that we describe and study in this section.

Definition 7.1. Let $k \in\{0, \ldots, n-1\}$. An elementary $k$-amalgamation of a regular simplex $\Delta=\Delta_{0} * \cdots * \Delta_{n}$ is the regular simplex: $\Delta^{\prime}=\Delta_{0}^{\prime} * \cdots * \Delta_{n-1}^{\prime}$ with

$$
\Delta_{i}^{\prime}= \begin{cases}\Delta_{i} & \text { if } i \leqslant n-k-2, \\ \Delta_{n-k-1} * \Delta_{n-k} & \text { if } i=n-k-1, \\ \Delta_{i-1} & \text { if } i \geqslant n-k .\end{cases}
$$

If $\Delta_{n-k-1}=\varnothing$ then the elementary amalgamation is $k$-simple.

We study the effect of these maps at the blow up level. First of all, we introduce a technical tool.

7.1. Stratification. The simplices $\Delta=\Delta_{0} * \cdots * \Delta_{n}$ and $\Delta^{\prime}=\Delta_{0}^{\prime} * \cdots * \Delta_{n-1}^{\prime}$ are filtered spaces (see Definition 1.1) of respective dimension $n$ and $n-1$. We set $\mathbb{N}_{\Delta}=\left\{i \in\{0, \ldots, n\} / \Delta_{0} * \cdots * \Delta_{n-i-1} \neq \Delta_{0} * \cdots * \Delta_{n-i}\right\}$. We observe that the family of strata of $\Delta$ is

$$
\mathcal{S}_{\Delta}=\left\{S_{i}=\Delta_{0} * \cdots * \Delta_{n-i} \backslash \Delta_{0} * \cdots * \Delta_{n-i-1} / i \in \mathbb{N}_{\Delta}\right\} .
$$


Notice that $\operatorname{codim} S_{i}=i$ for each $i \in \mathbb{N}_{\Delta}$.

If $k \in\{0, \ldots, n-1\}$, we denote $\varepsilon_{k}:\{0, \ldots, n\} \rightarrow\{0, \ldots, n-1\}$ the map defined by $\varepsilon_{k}(i)=i$ if $i \leqslant k$ and $\varepsilon_{k}(i)=i-1$ if $i \geqslant k+1$. We check easily that this maps restricts as a map $a_{k}: \mathbb{N}_{\Delta} \rightarrow \mathbb{N}_{\Delta^{\prime}}$, called the index map. If $S_{i} \in \mathcal{S}_{\Delta}$, we have $S_{i} \subset S_{a_{k}(i)}^{\prime}$. Thus by definition, the identity map is a stratified map from $\Delta$ to $\Delta^{\prime}$, we denote it

$$
\mathcal{A}[k]: \Delta=\Delta_{0} * \cdots * \Delta_{n} \rightarrow \Delta^{\prime}=\Delta_{0} * \cdots * \Delta_{n-1}^{\prime} .
$$

If $S \in \mathcal{S}_{\Delta}$, with the notations of Definition 1.5 we have $S^{\mathcal{A}[k]}=S_{a_{k}(i)}^{\prime}$, with $i=$ $\operatorname{codim} S$, and therefore

$$
\operatorname{codim} S^{\mathcal{A}[k]}=a_{k}(\operatorname{codim} S) .
$$

We also say that $\mathcal{A}[k]$ is an elementary amalgamation. If the elementary $k$ amalgamation is simple, then $a_{k}$ is a bijection and $\mathcal{A}[k]$ is a stratified homeomorphism (see Definition 1.5).

The elementary $k$-amalgamation has an effect only on two components . We first focus on them. For the sake of the simplicity we write $E_{0}=\Delta_{n-k-1}$ and $E_{1}=\Delta_{n-k}$, they can be empty.

Definition 7.2. We define a map

$$
\theta: N_{*}\left(\mathrm{c} E_{0}\right) \otimes N_{*}\left(\mathrm{c} E_{1}\right) \rightarrow N_{*}\left(\mathrm{c}\left(E_{0} * E_{1}\right)\right)
$$

by

$$
\theta\left(\left(F_{0}, \varepsilon_{0}\right) \otimes\left(F_{1}, \varepsilon_{1}\right)\right)= \begin{cases}\left(F_{0} * F_{1}, \varepsilon_{1}\right) & \text { if } \varepsilon_{0}=1, \\ \left(F_{0}, 0\right) & \text { if } \varepsilon_{0}=0 \text { and }\left|\left(F_{1}, \varepsilon_{1}\right)\right|=0, \\ 0 & \text { if not. }\end{cases}
$$

Notice that the restriction of $\theta$ to $N_{*}\left(\mathrm{c} E_{0}\right) \otimes N_{*}\left(E_{1}\right)$ gives a map, still denoted $\theta$,

$$
\theta: N_{*}\left(\mathrm{c} E_{0}\right) \otimes N_{*}\left(E_{1}\right) \rightarrow N_{*}\left(E_{0} * E_{1}\right) .
$$

By duality, we get a map

$$
\Xi: N^{*}\left(\mathrm{c}\left(E_{0} * E_{1}\right)\right) \rightarrow N^{*}\left(\mathrm{c} E_{0}\right) \otimes N^{*}\left(\mathrm{c} E_{1}\right)
$$

which verifies

$$
\left\{\begin{array}{lll}
\Xi\left(\mathbf{1}_{\left(F_{0} * F_{1}, \varepsilon\right)}\right)=(-1)^{\left|\left(F_{0}, 1\right)\right| \cdot\left|\left(F_{1}, \varepsilon\right)\right|} \mathbf{1}_{\left(F_{0}, 1\right)} \otimes \mathbf{1}_{\left(F_{1}, \varepsilon\right)} & \text { if }\left(F_{1}, \varepsilon\right) \neq(\varnothing, 0) . \\
\Xi\left(\mathbf{1}_{\left(F_{0}, 0\right)}\right)=\mathbf{1}_{\left(F_{0}, 0\right)} \otimes \lambda_{c E_{1}}, & \text { with } \lambda_{c E_{1}}=\mathbf{1}_{(\varnothing, 1)} \\
& & +\sum_{e \in \mathcal{V}\left(E_{1}\right)} \mathbf{1}_{(e, 0)} .
\end{array}\right.
$$

We also denote $\Xi: N^{*}\left(E_{0} * E_{1}\right) \rightarrow N^{*}\left(\mathrm{c} E_{0}\right) \otimes N^{*}\left(E_{1}\right)$ the restriction of $\Xi$.

LEMma 7.3. The morphisms $\theta$ and $\Xi$ are chain maps. Moreover,

(1) if $E_{0}=\varnothing$ then $\theta$ and $\Xi$ are isomorphisms,

(2) $\Xi$ is compatible with the cup products,

(3) $\theta$ and $\Xi$ are compatible with the cap product, i.e.,

$$
\begin{aligned}
\theta\left(\Xi\left(\mathbf{1}_{\left(F_{0} * F_{1}, \varepsilon\right)}\right) \widetilde{\cap}\left(\left[\mathrm{c} E_{0}\right] \otimes\left[\mathrm{c} E_{1}\right]\right)\right) & =\mathbf{1}_{\left(F_{0} * F_{1}, \varepsilon\right)} \cap\left[\mathrm{c}\left(E_{0} * E_{1}\right)\right], \text { and } \\
\theta\left(\Xi\left(\mathbf{1}_{F_{0} * F_{1}}\right) \widetilde{\cap}\left(\left[\mathrm{c} E_{0}\right] \otimes\left[E_{1}\right]\right)\right) & =\mathbf{1}_{F_{0} * F_{1}} \cap\left[E_{0} * E_{1}\right]
\end{aligned}
$$

where $[\nabla]$ is the maximal simplex of an euclidean simplex $\nabla,\left(F_{0} * F_{1}, \varepsilon\right)$, is a face of $\mathrm{c}\left(E_{0} * E_{1}\right)$ and $F_{0} * F_{1}$, is a face of $E_{0} * E_{1}$. 
We postpone the proof of this Lemma and deduce first some properties on amalgamations from it.

Definition 7.4. Let us consider the elementary $k$-amalgamation $\mathcal{A}[k]: \Delta \rightarrow$ $\Delta^{\prime}$ with $k \in\{0, \ldots, n-1\}$ (see Definition 7.1). We define two homomorphisms $\mathcal{A}[k]_{*}: \tilde{N}_{*}(\Delta) \rightarrow \tilde{N}_{*}\left(\Delta^{\prime}\right)$ and $\mathcal{A}[k]^{*}: \tilde{N}^{*}\left(\Delta^{\prime}\right) \rightarrow \tilde{N}^{*}(\Delta)$ by

$$
\mathcal{A}[k]_{*}=\underbrace{\mathrm{id} \otimes \cdots \otimes \mathrm{id}}_{n-k-1 \text { times }} \otimes \theta \otimes \underbrace{\mathrm{id} \otimes \cdots \otimes \mathrm{id}}_{k \text { times }}
$$

and

$$
\mathcal{A}[k]^{*}=\underbrace{\mathrm{id} \otimes \cdots \otimes \mathrm{id}}_{n-k-1 \text { times }} \otimes \Xi \otimes \underbrace{\mathrm{id} \otimes \cdots \otimes \mathrm{id}}_{k \text { times }}
$$

If there is not ambiguity, we use the notation $\mathcal{A}_{*}$ and $\mathcal{A}^{*}$ for these two maps and $a$ for the index map. In order to get coherent notation, we write $\|-\|_{0}=0$.

Proposition 7.5. Let $\mathcal{A}: \Delta \rightarrow \Delta^{\prime}$ be an elementary $k$-amalgamation. Then, the maps $\mathcal{A}_{*}$ and $\mathcal{A}^{*}$ defined above satisfy the following properties.

(1) They are chain maps and $\mathcal{A}^{*}$ is compatible with the cup product.

(2) Let $\omega \in \tilde{N}^{*}\left(\Delta^{\prime}\right)$, then we have

$$
\mathcal{A}_{*}\left(\mathcal{A}^{*}(\omega) \widetilde{\cap} \widetilde{\Delta}\right)=\omega \widetilde{\cap} \widetilde{\Delta^{\prime}} .
$$

(3) In the case of simple amalgamations, they are isomorphisms.

(4) For each regular face operator $\delta_{\ell}: \nabla \rightarrow \Delta$ we denote by $\mathcal{A}_{\Delta}^{*}$ the previous map and by $\mathcal{A}_{\nabla}^{*}$ the map corresponding to the amalgamation induced on $\nabla$. Then we have

$$
\delta_{\ell}^{*} \circ \mathcal{A}_{\nabla}^{*}=\mathcal{A}_{\Delta}^{*} \circ \delta_{\ell}^{*} .
$$

(5) The map $\mathcal{A}^{*}$ decreases the perverse degree, i.e., for $\omega \in \tilde{N}^{*}\left(\Delta^{\prime}\right)$ and $\ell \in \mathbb{N}_{\Delta}$, we have

$$
\left\|\mathcal{A}^{*}(\omega)\right\|_{\ell} \leqslant\|\omega\|_{a(\ell)} .
$$

Moreover, if the amalgamation is simple, this inequality becomes an equality,

$$
\left\|\mathcal{A}^{*}(\omega)\right\|_{\ell}=\|\omega\|_{a(\ell)} .
$$

Proof. The properties (1) and (3) are consequences of Lemma 7.3 (2),(1).

(2) From the definition of $\mathcal{A}_{*}$ and $\mathcal{A}^{*}$ it suffices to apply Lemma 7.3 (3).

(4) Direct from definitions.

(5) Let $\omega=\mathbf{1}_{\left(F_{0}, \varepsilon_{0}\right)} \otimes \cdots \otimes \mathbf{1}_{\left(F_{n-k-2}, \varepsilon_{n-k-2}\right)} \otimes \mathbf{1}_{\left(F_{n-k-1} * F_{n-k}, \varepsilon\right)} \otimes \mathbf{1}_{\left(F_{n-k+1}, \varepsilon_{n-k+1}\right)} \otimes$ $\cdots \otimes 1_{F_{n}} \in \tilde{N}^{*}\left(\Delta^{\prime}\right)$. We distinguish two cases (see Definitions 7.2 7.4):

- Suppose $\left(F_{n-k}, \varepsilon\right) \neq(\varnothing, 0)$. We have, up to a sign, $\mathcal{A}^{*}(\omega)= \pm \mathbf{1}_{\left(F_{0}, \varepsilon_{0}\right)} \otimes$ $\cdots \otimes \mathbf{1}_{\left(F_{n-k-2}, \varepsilon_{n-k-2}\right)} \otimes \mathbf{1}_{\left(F_{n-k-1}, 1\right)} \otimes \mathbf{1}_{\left(F_{n-k}, \varepsilon\right)} \otimes \mathbf{1}_{\left(F_{n-k+1}, \varepsilon_{n-k+1}\right)} \otimes \cdots \otimes$ $\mathbf{1}_{\left(F_{n}, \varepsilon_{n}\right)}$. The result comes from

$$
\left\|\mathcal{A}^{*}(\omega)\right\|_{\ell}=\left\{\begin{array}{cl}
\|\omega\|_{\ell-1} & \text { if } \ell \geqslant k+2 \\
-\infty & \text { if } \ell=k+1 \\
\|\omega\|_{\ell} & \text { if } \ell \leqslant k
\end{array} \leqslant\left\{\begin{array}{cl}
\|\omega\|_{\ell-1} & \text { if } \ell \geqslant k+1 \\
\|\omega\|_{\ell} & \text { if } \ell \leqslant k
\end{array}=\|\omega\|_{a(\ell)},\right.\right.
$$

for $\ell \in \mathbb{N}_{\Delta}$. 
- Suppose $\left(F_{n-k}, \varepsilon\right)=(\varnothing, 0)$. We have the equality $\mathcal{A}^{*}(\omega)=\mathbf{1}_{\left(F_{0}, \varepsilon_{0}\right)} \otimes \cdots \otimes$ $\mathbf{1}_{\left(F_{n-k-2}, \varepsilon_{k-2}\right)} \otimes \mathbf{1}_{\left(F_{n-k-1}, 0\right)} \otimes \mathbf{1}_{(\varnothing, 1)} \otimes \mathbf{1}_{\left(F_{n-k+1}, \varepsilon_{n-k+1}\right)} \otimes \cdots \otimes \mathbf{1}_{\left(F_{n}, \varepsilon_{n}\right)}+$ $\sum_{e \in \mathcal{V}\left(\Delta_{k}\right)} \mathbf{1}_{\left(F_{0}, \varepsilon_{0}\right)} \otimes \cdots \otimes \mathbf{1}_{\left(F_{n-k-2}, \varepsilon_{n-k-2}\right)} \otimes \mathbf{1}_{\left(F_{n-k-1}, 0\right)} \otimes \mathbf{1}_{(e, 0)} \otimes$ $\mathbf{1}_{\left(F_{n-k+1}, \varepsilon_{n-k+1}\right)} \otimes \cdots \otimes \mathbf{1}_{\left(F_{n}, \varepsilon_{n}\right)}$. Then we have

$$
\left\|\mathcal{A}^{*}(\omega)\right\|_{\ell}=\left\{\begin{array}{ll}
\|\omega\|_{\ell-1} & \text { if } \ell \geqslant k+2 \\
\|\omega\|_{\ell} & \text { if } \ell \leqslant k
\end{array}=\|\omega\|_{a(\ell)}\right.
$$

for $\ell \in \mathbb{N}_{\Delta}$. Since the amalgamation is simple, the equality comes from the fact that $k+1 \notin \mathbb{N}_{\Delta}$.

Proof of Lemma 7.3. We begin with the compatibility with the differentials. Let $H=\left(F_{0}, \varepsilon_{0}\right) \otimes\left(F_{1}, \varepsilon_{1}\right)$ and suppose $\varepsilon_{0}=1$ and $\left|\left(F_{1}, \varepsilon_{1}\right)\right| \geqslant 2$. We have

$$
\begin{aligned}
\theta \partial H= & \theta\left(\left(\partial F_{0}, 1\right) \otimes\left(F_{1}, \varepsilon_{1}\right)\right)+(-1)^{\left|\left(F_{0}, 1\right)\right|} \theta\left(\left(F_{0}, 1\right) \otimes\left(\partial F_{1}, \varepsilon_{1}\right)\right) \\
& +(-1)^{\left|\left(F_{0}, 1\right)+\right| F_{1} \mid+1} \begin{cases}0 & \text { if } \varepsilon_{1}=0 \\
\theta\left(\left(F_{0}, 1\right) \otimes\left(F_{1}, 0\right)\right) & \text { if } \varepsilon_{1}=1\end{cases} \\
= & \left(\partial F_{0} * F_{1}, \varepsilon_{1}\right)+(-1)^{\left|\left(F_{0}, 1\right)\right|}\left(F_{0} * \partial F_{1}, \varepsilon_{1}\right) \\
& + \begin{cases}0 & \text { if } \varepsilon_{1}=0 \\
(-1)^{\left|\left(F_{0}, 1\right)+\right| F_{1} \mid+1}\left(F_{0} * F_{1}, 0\right) & \text { if } \varepsilon_{1}=1\end{cases} \\
= & \partial\left(F_{0} * F_{1}, \varepsilon_{1}\right)
\end{aligned}
$$

On the other hand, we get $\partial \theta H=\partial\left(F_{0} * F_{1}, \varepsilon_{1}\right)$ and we have established the equality $\theta \partial=\partial \theta$ in the case $\varepsilon_{0}=1$ and $\left|\left(F_{1}, \varepsilon_{1}\right)\right| \geqslant 2$. The proof is similar in the other cases. The compatibility of $\Xi$ with the differentials follows by duality.

(1) If $E_{0}=\varnothing$, then $\varepsilon_{0}=1$ and, by definition of the map $\theta$, we have $\theta((\varnothing, 1) \otimes$ $\left.\left(F_{1}, \varepsilon_{1}\right)\right)=\left(F_{1}, \varepsilon_{1}\right)$. Therefore, $\theta$ is an isomorphism and so is $\Xi$ by duality.

(2) Let $\mathbf{1}_{\left(F_{0} * F_{1}, \varepsilon\right)}, \mathbf{1}_{\left(F_{0}^{\prime} * F_{1}^{\prime}, \varepsilon^{\prime}\right)} \in \tilde{N}^{*}\left(\mathrm{c}\left(E_{0} * E_{1}\right)\right)$. We suppose that $\left(F_{1}, \varepsilon\right)$ and $\left(F_{1}^{\prime}, \varepsilon^{\prime}\right)$ are different from $(\varnothing, 0)$. Let us consider $A=\Xi\left(\mathbf{1}_{\left(F_{0} * F_{1}, \varepsilon\right)}\right) \cup \Xi\left(\mathbf{1}_{\left(F_{0}^{\prime} * F_{1}^{\prime}, \varepsilon^{\prime}\right)}\right)$ and $B=\Xi\left(\mathbf{1}_{\left(F_{0} * F_{1}, \varepsilon\right)} \cup \mathbf{1}_{\left(F_{0}^{\prime} * F_{1}^{\prime}, \varepsilon^{\prime}\right)}\right)$. A direct computation gives the following equalities from definitions.

$$
\begin{array}{r}
A=(-1)^{\left|\left(F_{0}, 1\right)\right| \cdot\left|\left(F_{1}, \varepsilon\right)\right|+\left|\left(F_{0}^{\prime}, 1\right)\right| \cdot\left|\left(F_{1}^{\prime}, \varepsilon^{\prime}\right)\right|}\left(\mathbf{1}_{\left(F_{0}, 1\right)} \otimes \mathbf{1}_{\left(F_{1}, \varepsilon\right)}\right) \cup\left(\mathbf{1}_{\left(F_{0}^{\prime}, 1\right)} \otimes \mathbf{1}_{\left(F_{1}^{\prime}, \varepsilon^{\prime}\right)}\right)= \\
(-1)^{\left|\left(F_{1}, \varepsilon\right)\right| \cdot\left|\left(F_{0}^{\prime}, 1\right)\right|+\left|\left(F_{0}, 1\right)\right| \cdot\left|\left(F_{1}, \varepsilon\right)\right|+\left|\left(F_{0}^{\prime}, 1\right)\right| \cdot\left|\left(F_{1}^{\prime}, \varepsilon^{\prime}\right)\right|}\left(\mathbf{1}_{\left(F_{0}, 1\right)} \cup \mathbf{1}_{\left(F_{0}^{\prime}, 1\right)}\right) \otimes\left(\mathbf{1}_{\left(F_{1}, \varepsilon\right)} \cup \mathbf{1}_{\left(F_{1}^{\prime}, \varepsilon^{\prime}\right)}\right)
\end{array}
$$

If $\left(F_{0}^{\prime}, \varepsilon\right)=(\varnothing, 0)$ and $F_{1}, F_{1}^{\prime}$ are compatibles (see Definition 4.1), we have

$$
\begin{aligned}
A & =(-1)^{\left|\left(F_{1}, 0\right)\right|\left(\left|\left(F_{0}, 1\right)\right|+\left|\left(F_{1}^{\prime}, \varepsilon^{\prime}\right)\right|\right)} \mathbf{1}_{\left(F_{0}, 1\right)} \otimes \mathbf{1}_{\left(F_{1} \cup F_{1}^{\prime}, \varepsilon^{\prime}\right)} \\
& =(-1)^{\left|\left(F_{0} * F_{1}, 0\right)\right| \cdot\left|\left(F_{1}^{\prime}, \varepsilon^{\prime}\right)\right|} \Xi\left(\mathbf{1}_{\left(F_{0} * F_{1} \cup F_{1}^{\prime}, \epsilon^{\prime}\right)}\right)=B .
\end{aligned}
$$

If $\left(F_{0}^{\prime}, \varepsilon\right)=(\varnothing, 1)$ and $\left(F_{1}^{\prime}, \varepsilon^{\prime}\right)=(\varnothing, 1)$, we have $A=(-1)^{\left|\left(F_{0}, 1\right)\right| \cdot\left|\left(F_{1}, 1\right)\right|} \mathbf{1}_{\left(F_{0}, 1\right)} \otimes$ $\mathbf{1}_{\left(F_{1}, 1\right)}=\Xi\left(\mathbf{1}_{\left(F_{0} * F_{1}, 1\right)}\right)=B$. In the other cases we have $A=B=0$.

We have established the equality $A=B$ in the case where $\left(F_{1}, \varepsilon\right)$ and $\left(F_{1}^{\prime}, \varepsilon^{\prime}\right)$ are different from $(\varnothing, 0)$. The verification is similar in the other cases. 
(3) Suppose $\left(F_{1}, \varepsilon\right) \neq(\varnothing, 0)$. We have

$$
\begin{aligned}
C & =\theta\left(\Xi\left(\mathbf{1}_{\left(F_{0} * F_{1}, \varepsilon\right)}\right) \widetilde{\cap}\left(\left[\mathrm{c} E_{0}\right] \otimes\left[\mathrm{c} E_{1}\right]\right)\right) \\
& =(-1)^{\left|\left(F_{0}, 1\right)\right| \cdot\left|\left(F_{1}, \varepsilon\right)\right|} \theta\left(\left(\mathbf{1}_{\left(F_{0}, 1\right)} \otimes \mathbf{1}_{\left(F_{1}, \varepsilon\right)}\right) \widetilde{\cap}\left(\left[\mathrm{c} E_{0}\right] \otimes\left[\mathrm{c} E_{1}\right]\right)\right) \\
& =(-1)^{\left|\left(F_{1}, \varepsilon\right)\right| \cdot\left|c E_{0}\right|+\left|\left(F_{0}, 1\right)\right| \cdot\left|\left(F_{1}, \varepsilon\right)\right|} \theta\left(\left(\mathbf{1}_{\left(F_{0}, 1\right)} \cap\left[\mathrm{c} E_{0}\right]\right) \otimes\left(\mathbf{1}_{\left(F_{1}, \varepsilon\right)} \cap\left[\mathrm{c} E_{1}\right]\right)\right) .
\end{aligned}
$$

We apply the definition of cap product (6.2) and we consider three different cases.

- If $F_{0}=E_{0}, F_{1}=E_{1}$ and $\varepsilon=1$, we have

$$
C=\theta([\mathrm{v}] \otimes[\mathrm{v}])=[\mathrm{v}]=\mathbf{1}_{\left(F_{0} * F_{1}, \varepsilon\right)} \cap\left[\mathrm{c}\left(E_{0} * E_{1}\right)\right] .
$$

- If $F_{0}=E_{0}, F_{1} \cup G_{1}=E_{1}$ and $\varepsilon=0$, we have

$$
C=\theta\left([\mathrm{v}] \otimes\left(G_{1}, 1\right)\right)=\left(G_{1}, 1\right)=\mathbf{1}_{\left(F_{0} * F_{1}, \varepsilon\right)} \cap\left[\mathrm{c}\left(E_{0} * E_{1}\right)\right] .
$$

- The other cases correspond to $C=\mathbf{1}_{\left(F_{0} * F_{1}, \varepsilon\right)} \cap\left[\mathrm{c}\left(E_{0} * E_{1}\right)\right]=0$.

We have established the property $(3)$ in the case $\left(F_{1}, \varepsilon\right) \neq(\varnothing, 0)$. The verification is similar in the other cases.

\section{Stratified maps: the global level. Theorem $\mathrm{A}$.}

Let $f: X \rightarrow Y$ be a stratified map, $\sigma: \Delta_{\sigma} \rightarrow X$ and $f \circ \sigma: \Delta_{f \circ \sigma} \rightarrow Y$ filtered simplices of $X$ and $Y$ respectively. In [4, Corollary A.25] we have proved that the filtrations $\Delta_{\sigma}$ and $\Delta_{f \circ \sigma}$ (of the same euclidean simplex $\Delta$ ) are connected by an amalgamation, more exactly, by a finite sequence $\mathcal{A}_{1}$ of elementary amalgamations and a finite sequence $\mathcal{A}_{2}^{-1}$ of inverse of simple amalgamations as follows.

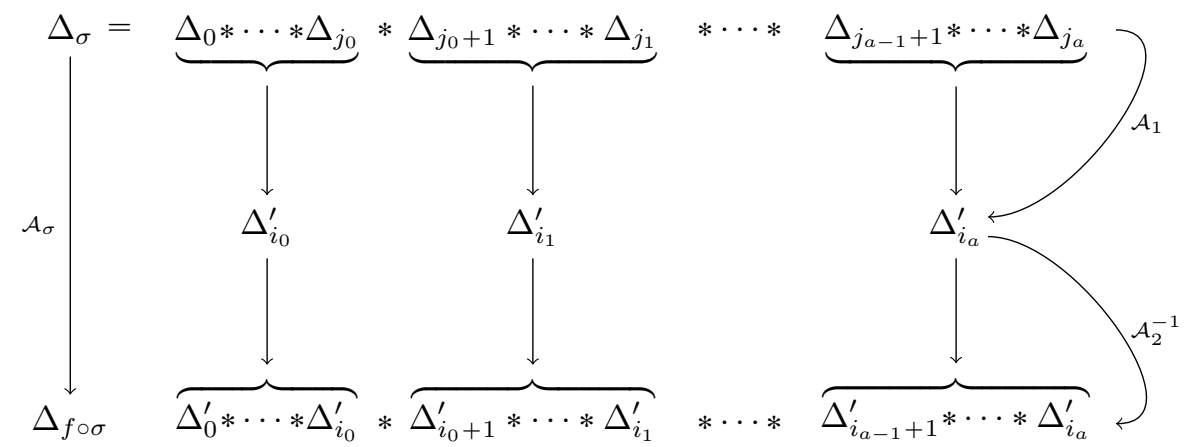

We denote the amalgamation $\mathcal{A}_{\sigma}=\mathcal{A}_{2}^{-1} \circ \mathcal{A}_{1}$, with $\mathcal{A}_{1}=\mathcal{A}_{1,1} \circ \cdots \circ \mathcal{A}_{1, u}$ and $\mathcal{A}_{2}=\mathcal{A}_{2,1} \circ \cdots \circ \mathcal{A}_{2, v}$. The elementary amalgamations $\mathcal{A}_{1, i}$ (resp. simple amalgamations $\mathcal{A}_{2, j}$ ) are written in a canonical way, going from the left to the right.

8.1. $\mu$-amalgamation. Mention the amalgamation of $\Delta_{\sigma}$ collecting all the filtration in one factor. We call it the $\mu$-amalgamation $\mathcal{A}_{\sigma}^{\prime \prime}: \Delta_{\sigma} \rightarrow \Delta$. The induced $\operatorname{map} \mathcal{A}_{\sigma, *}^{\prime \prime}: \tilde{N}_{*}\left(\Delta_{\sigma}\right) \rightarrow N_{*}(\Delta)$ is defined by

$\bigotimes_{i=0}^{n}\left(F_{i}, \varepsilon_{i}\right) \quad \leadsto \quad\left(F_{0} * \cdots * F_{\ell}, 0\right) \otimes \bigotimes_{i>\ell}\left(F_{i}, \varepsilon_{i}\right) \leadsto\left\{\begin{array}{cl}F_{0} * \cdots * F_{\ell} & \text { if }|(F, \epsilon)|_{>\ell}=0 \\ 0 & \text { otherwise }\end{array}\right.$

with $\varepsilon_{n}=0$ and $\ell$ is the smallest integer, $j$, such that $\varepsilon_{j}=0$ (see Definition 6.1). We recover the map $\mu_{*}$ of (6.4). We also define $\mu^{*}=\mathcal{A}_{\sigma}^{\prime \prime *}$. 
With the previous notations, we notice the commutativity of the following diagram

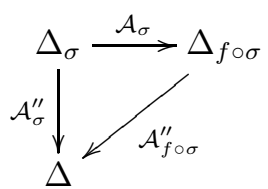

If $\bar{p}$ is a perversity on $X$ and $\bar{q}$ is a perversity on $Y$ with $\bar{p} \leqslant f^{*} \bar{q}$, we have proved in [5. Proposition 3.6] that the map $f$ induces a homomorphism $f_{*}: H_{*}^{\bar{p}}(X ; R) \rightarrow$ $H_{*}^{\bar{p}}(Y ; R)$. We define now the induced map in blown-up intersection cohomology and study its properties.

Definition 8.1. Let $f: X \rightarrow Y$ be a stratified map. The induced map $f: \tilde{N}^{*}(Y ; R) \rightarrow \tilde{N}^{*}(X ; R)$ is the map defined by $\left(f^{*} \omega\right)_{\sigma}=\mathcal{A}_{\sigma}^{*} \omega_{f \circ \sigma}$, for each $\omega \in \tilde{N}^{*}(Y ; R)$ and each regular simplex $\sigma: \Delta_{\sigma} \rightarrow X$.

With Proposition 7.5) (4) this definition makes sense. It generalizes the notion of induced map of Proposition 3.5 (see Remark 3.6).

TheOrem A. Let $f: X \rightarrow Y$ be a stratified map.

(1) The induced morphism $f^{*}: \tilde{N}^{*}(Y ; R) \rightarrow \tilde{N}^{*}(X ; R)$ is a chain map.

(2) The map $f^{*}$ is compatible with the cup product.

(3) The maps $f_{*}: \mathfrak{C}_{*}(X ; R) \rightarrow \mathfrak{C}_{*}(Y ; R)$ and $f^{*}: \tilde{N}^{*}(Y ; R) \rightarrow \tilde{N}^{*}(X ; R)$ verify

$$
f_{*}\left(f^{*} \omega \cap \xi\right)=\omega \cap f_{*} \xi
$$

for each $\omega \in \tilde{N}^{*}(Y ; R)$ and each $\xi \in \mathfrak{C}_{*}(X ; R)$.

(4) Let $\bar{p}$ be a perversity on $X$ and $\bar{q}$ a perversity on $Y$ such that $\bar{p} \geqslant f^{*} \bar{q}$. Then

$f$ induces a chain map $f^{*}: \tilde{N}_{\bar{q}}^{*}(Y ; R) \rightarrow \tilde{N}_{\bar{p}}^{*}(X ; R)$, thus a homomorphism $f^{*}: \mathscr{H}_{\bar{q}}^{*}(X ; R) \rightarrow \mathscr{H}_{\bar{p}}^{*}(Y ; R)$.

As a GM-perversity verifies $\bar{p} \geqslant f^{*} \bar{p}$, the previous Theorem has an adaptation in this context.

COROllary 8.2. Let $f: X \rightarrow Y$ be a stratified map and $\bar{p}$ a GM-perversity. Then $f$ induces a homomorphism $f^{*}: \mathscr{H}_{\bar{p}}^{*}(Y ; R) \rightarrow \mathscr{H}_{\bar{p}}^{*}(X ; R)$ compatible with cup products.

The maps induced in cohomology and homology for a GM-perversity satisfy also the condition (3) of Theorem $\mathrm{A}$.

Proof of Theorem A, Properties (1) and (2) are direct consequences of Proposition 7.5

(3) Notice first that $f_{*}: \mathfrak{C}_{*}(X ; R) \rightarrow \mathfrak{C}_{*}(Y ; R)$ is well defined since $f_{*}$ preserves filtered simplices (see [4, Theorem F]) and $f\left(X \backslash \Sigma_{X}\right) \subset Y \backslash \Sigma_{Y}$, since $f$ is stratified.

We can suppose that $\xi$ is a regular simplex $\sigma: \Delta_{\sigma} \rightarrow X$. Applying Proposition $7.5(2)$ repeatedly we have $\mathcal{A}_{\sigma, *}\left(\mathcal{A}_{\sigma}^{*} \omega_{f \circ \sigma} \tilde{\cap} \tilde{\Delta}_{\sigma}\right)=\omega_{f \circ \sigma} \widetilde{\cap} \tilde{\Delta}_{f \circ \sigma}$. The result follows from

$$
\begin{array}{r}
f_{*}\left(f^{*} \omega \cap \sigma\right)=f_{*} \sigma_{*} \mu_{\Delta_{\sigma}, *}\left(\left(f^{*} \omega\right)_{\sigma} \widetilde{\cap} \widetilde{\Delta}_{\sigma}\right)=f_{*} \sigma_{*} \mu_{\Delta_{\sigma}, *}\left(\mathcal{A}_{\sigma}^{*} \omega_{f \circ \sigma} \widetilde{\cap} \tilde{\Delta}_{\sigma}\right) \\
\stackrel{\text { 8.1 }}{=} f_{*} \sigma_{*} \mu_{\Delta_{f \circ \sigma}, *} \mathcal{A}_{\sigma, *}\left(\mathcal{A}_{\sigma}^{*} \omega_{f \circ \sigma} \widetilde{\cap} \widetilde{\Delta}_{\sigma}\right)=(f \circ \sigma)_{*} \mu_{\Delta_{f \circ \sigma}, *}\left(\omega_{f \circ \sigma} \widetilde{\cap} \widetilde{\Delta}_{f \circ \sigma}\right) \\
=\omega \cap f_{*}(\sigma) .
\end{array}
$$


(4) Let $\omega \in \tilde{N}_{\bar{q}}^{*}(Y ; R)$. Let $\sigma: \Delta \rightarrow X$ be a regular simplex and $S$ a singular stratum of $X$ with $\operatorname{Im} \sigma \cap S \neq \varnothing$. We have to prove

$$
\|\omega\|_{S^{f}} \leqslant \bar{q}\left(S^{f}\right) \Rightarrow\left\|f^{*} \omega\right\|_{S} \leqslant \bar{p}(S)
$$

Since $\bar{p} \geqslant f^{*} \bar{q}$, it suffices to prove $\left\|\mathcal{A}_{\sigma}^{*} \omega_{f \circ \sigma}\right\|_{\operatorname{codim} S} \leqslant\left\|\omega_{f \circ \sigma}\right\|_{\operatorname{codim} S^{f}}$. Recall the decomposition, $\mathcal{A}_{\sigma}=\mathcal{A}_{2, v}^{-1} \circ \cdots \circ \mathcal{A}_{2,1}^{-1} \circ \mathcal{A}_{1,1} \circ \cdots \circ \mathcal{A}_{1, u}$, at the beginning of the section and denote by $a_{1, i}, a_{2, j}$ the associated index maps. We have

$$
\operatorname{codim} S^{f}=\left(a_{2, v}^{-1} \circ \cdots \circ a_{2,1}^{-1} \circ a_{1,1} \circ \cdots \circ a_{1, u}\right)(\operatorname{codim} S)
$$

(see (17.1)). Now, the result follows from Proposition 7.5 (5).

\section{Part 2. Properties of the Blown-up intersection cohomology}

We present the properties of the blown-up intersection cohomology used in this work. We end this Part by comparing this cohomology with the intersection cohomology obtained from the dual cochains.

\section{9. $\mathcal{U}$-small chains. Theorem $\mathrm{B}$.}

We establish a Theorem of $\mathcal{U}$-small filtered simplices, where $\mathcal{U}$ is an open cover of $X$ (Theorem B).

Let $\Delta$ be an euclidean simplex whose vertices are $e_{0}, \ldots, e_{m}$. Let $e_{\left\{i_{0} \ldots i_{p}\right\}}$ be the barycenter of the simplex $\left[e_{i_{0}}, \ldots, e_{i_{p}}\right]$ and $\operatorname{Sub} \Delta$ the simplicial complex given by the barycentric subdivision of $\Delta$. The subdivision linear map, $\operatorname{Sub}_{*}: N_{*}(\Delta) \rightarrow$ $N_{*}(\operatorname{Sub} \Delta)$, is defined by $\operatorname{Sub}_{*}\left[e_{i_{0}}, \ldots, e_{i_{p}}\right]=(-1)^{p}\left(\operatorname{Sub}_{*} \partial\left[e_{i_{0}}, \ldots, e_{i_{p}}\right]\right) * e_{\left\{i_{0} \ldots i_{p}\right\}}$. Any face of Sub $\Delta$ appears at most once in the expression of $\operatorname{Sub}_{*}$ and some faces do not.

Definition 9.1. An $\ell$-simplex of $\operatorname{Sub} \Delta$ is a subdividing simplex if it appears in the subdivision of an $\ell$-simplex of $\Delta$.

From the definition of $\mathrm{Sub}_{*}$ we know that the subdividing simplices of $\mathrm{Sub} \Delta$ are the simplices of the form

$$
\nabla=\left[e_{i_{0}}, e_{\left\{i_{0} i_{1}\right\}}, e_{\left\{i_{0} i_{1} i_{2}\right\}}, \ldots, e_{\left\{i_{0} \ldots i_{p}\right\}}\right] .
$$

The vertex $e_{i_{0}}$ is the first vertex and the vertex $e_{i_{p}}$ is the last vertex of $\nabla$. We write $e_{i_{p}}=\operatorname{der} \nabla$.

The transpose map of $\mathrm{Sub}_{*}$ is denoted by $\mathrm{Sub}^{*}: N^{*}(\operatorname{Sub} \Delta) \rightarrow N^{*}(\Delta)$. It is defined on the subdividing simplices by

$$
\operatorname{Sub}^{*}\left(\mathbf{1}_{\left[e_{i_{0}}, e_{\left\{i_{0} i_{1}\right\}}, e_{\left\{i_{0} i_{1} i_{2}\right\}}, \ldots, e_{\left\{i_{0} \ldots i_{p}\right\}}\right]}\right)=\mathbf{1}_{\left[e_{i_{0}}, \ldots, e_{i_{p}}\right]},
$$

and by 0 on the other simplices. Since the linear map $\mathrm{Sub}_{*}$ is compatible with differentials, then Sub* also is a cochain map.

To construct a homotopy between the barycentric subdivision operator and the identity, we consider the simplicial complex $K(\Delta)$, whose simplices are the joins, $F * G$, of a simplex $F$ of $\Delta$ and of a simplex $G$ of Sub $\Delta$, such that: if $F=$ $\left[e_{i_{0}}, \ldots, e_{i_{p}}\right]$ and $G=\left[e_{J_{0}}, \ldots, e_{J_{k}}\right]$, then $\left\{i_{0}, \ldots, i_{p}\right\} \subset J_{\ell}$, for each $\ell \in\{0, \ldots, k\}$. In addition, simplices $F$ and $G$ can be empty, but not simultaneously, making $\Delta$ and Sub $\Delta$ two sub-complexes of $K(\Delta)$. 
The homotopy operator, $T: N_{*}(\Delta) \rightarrow N_{*+1}(K(\Delta))$, defined by $T\left[e_{i_{0}}, \ldots, e_{i_{p}}\right]=(-1)^{p+1}\left(\left[e_{i_{0}}, \ldots, e_{i_{p}}\right]-T \partial\left[e_{i_{0}}, \ldots, e_{i_{p}}\right]\right) * e_{\left\{i_{0} \ldots i_{p}\right\}}$, verifies

$$
(\partial T+T \partial)(F)=F-\operatorname{Sub}_{*}(F) .
$$

In particular, $T$ takes the values, $T\left(e_{0}\right)=-e_{0} * e_{\{0\}}$ and $T\left[e_{0}, e_{1}\right]=\left[e_{0} e_{1}\right] * e_{\{01\}}-$ $e_{0} *\left[e_{\{0\}}, e_{\{0,1\}}\right]+e_{1} *\left[e_{\{1\}}, e_{\{0,1\}}\right]$.

Definition 9.2. A full simplex of $K(\Delta)$ is a simplex of the form

$$
F * G=\left[e_{i_{0}}, \ldots, e_{i_{p}}\right] *\left[e_{\left\{i_{0} \ldots i_{p}\right\}}, e_{\left\{i_{0} \ldots i_{p} i_{p+1}\right\}}, \ldots, e_{\left\{i_{0} \ldots i_{p} \ldots i_{p+r}\right\}}\right] .
$$

The vertex $e_{i_{p+r}}$ is the last vertex of $F * G$ and denoted by $\operatorname{der}(F * G)$.

Note that if $F * G$ is full and $p=0$, then $G$ is subdividing in the sense of Definition 9.1

Endow the simplex $\Delta$ with a filtration, $\Delta=\Delta_{0} * \cdots * \Delta_{n}$, compatible with the order considered on the vertices, cf. (2.2). The simplicial complex Sub $\Delta$ is a weighted simplicial complex, associating to the vertex $e_{\left\{i_{0} \ldots i_{p}\right\}}$ the weight $\ell$ if we have $\left[e_{i_{0}}, \ldots, e_{i_{p}}\right] \triangleleft \Delta_{0} * \cdots * \Delta_{\ell}$ and $\left[e_{i_{0}}, \ldots, e_{i_{p}}\right] \pitchfork \Delta_{0} * \cdots * \Delta_{\ell-1}$. Since the family of the vertices of $K(\Delta)$ is the union of those of $\Delta$ and those of Sub $\Delta$, the complex $K(\Delta)$ also is a weighted simplicial complex. The chosen canonical basis of $\tilde{N}^{*}(K(\Delta))$ is composed of elements of the form,

$$
\mathbf{1}_{(F * G, \varepsilon)}=\mathbf{1}_{\left(F_{0}, \varepsilon_{0}\right)} \otimes \cdots \otimes \mathbf{1}_{\left(F_{q} * G_{q}, \varepsilon_{q}\right)} \otimes \cdots \otimes \mathbf{1}_{\left(G_{n-1}, \varepsilon_{n-1}\right)} \otimes \mathbf{1}_{G_{n}},
$$

with $F=F_{0} * \cdots * F_{q} \triangleleft \Delta$ and $G=G_{q} * \cdots * G_{n} \triangleleft \operatorname{Sub} \Delta$.

REMARK 9.3. The linear operator Sub* does not necessarily respect the filter degree of simplices. Consider $\Delta=\left[e_{0}\right] *\left[e_{1}, e_{2}\right]$. Previous computations give $\operatorname{Sub}^{*}\left(\mathbf{1}_{\left[e_{1}, e_{\{0,1\}}\right]}\right)=\mathbf{1}_{\left[e_{1}, e_{0}\right]}$. Taking into account the filtration defined on Sub $\Delta$, one has $\left[e_{1}, e_{\{0,1\}}\right]=\varnothing *\left[e_{1}, e_{\{0,1\}}\right]$ and $\left[e_{1}, e_{0}\right]=-\left[e_{0}\right] *\left[e_{1}\right]$, from which one gets $\operatorname{Sub}^{*}\left(\mathbf{1}_{\varnothing *\left[e_{1}, e_{\{0,1\}}\right]}\right)=-\mathbf{1}_{\left[e_{0}\right] *\left[e_{1}\right]}$.

More generally, let $\Delta=\Delta_{0} * \cdots * \Delta_{n}$ and let $\nabla=\nabla_{0} * \cdots * \nabla_{n} \triangleleft \operatorname{Sub} \Delta$. The previous example shows that the use of $\mathrm{Sub}^{*}$ for the construction of a cochain morphism,

$\widetilde{\mathrm{Sub}}: N^{*}\left(\mathrm{c} \nabla_{0}\right) \otimes \cdots \otimes N^{*}\left(\mathrm{c} \nabla_{n-1}\right) \otimes N^{*}\left(\nabla_{n}\right) \rightarrow N^{*}\left(\mathrm{c} \Delta_{0}\right) \otimes \cdots \otimes N^{*}\left(\mathrm{c} \Delta_{n-1}\right) \otimes N^{*}\left(\Delta_{n}\right)$, requires a reordering of the vertices according to the filtrations of $\Delta$ and $\operatorname{Sub} \Delta$. To avoid this difficulty we define below the morphism $\widetilde{\operatorname{Sub}}_{\Delta}: \tilde{N}^{*}(\operatorname{Sub} \Delta) \rightarrow \tilde{N}^{*}(\Delta)$ and the homotopy $\widetilde{\mathrm{T}}_{\Delta}: \tilde{N}^{*}(K(\Delta)) \rightarrow \tilde{N}^{*-1}(\Delta)$ by induction.

The two canonical injections of $\Delta$ and Sub $\Delta$ into $K(\Delta)$ induce the chain complex epimorphisms,

- $\iota_{\Delta}^{*}: \tilde{N}^{*}(K(\Delta)) \rightarrow \tilde{N}^{*}(\Delta)$, defined by the identity on the elements $\mathbf{1}_{(F, \varepsilon)}$ with $F \triangleleft \Delta$ and 0 elsewhere,

- $\iota_{\text {Sub } \Delta}^{*}: \tilde{N}^{*}(K(\Delta)) \rightarrow \tilde{N}^{*}(\operatorname{Sub} \Delta)$, defined by the identity on the elements $\mathbf{1}_{(G, \varepsilon)}$ with $G \triangleleft \operatorname{Sub} \Delta$ and 0 elsewhere.

Lemma 9.4. There exist a linear map, $\widetilde{\mathrm{T}}_{\Delta}: \tilde{N}^{*}(K(\Delta)) \rightarrow \tilde{N}^{*-1}(\Delta)$, and a complex cochain morphism, $\widetilde{\operatorname{Sub}}_{\Delta}: \tilde{N}^{*}(\operatorname{Sub} \Delta) \rightarrow \tilde{N}^{*}(\Delta)$, verifying

$$
\widetilde{\mathrm{T}}_{\Delta} \circ \widetilde{\delta}^{K(\Delta)}+\widetilde{\delta}^{\Delta} \circ \widetilde{\mathrm{T}}_{\Delta}=\iota_{\Delta}^{*}-\widetilde{\operatorname{Sub}} \Delta \circ \iota_{\mathrm{Sub} \Delta}^{*},
$$

where $\widetilde{\delta}^{K(\Delta)}$ and $\widetilde{\delta}^{\Delta}$ are the differentials on $\tilde{N}^{*}(K(\Delta))$ and $\tilde{N}^{*}(\Delta)$. 
9.1. Construction of $\widetilde{\mathrm{Sub}} \Delta$ and $\widetilde{\mathrm{T}}_{\Delta}$. The homotopy $\widetilde{\mathrm{T}}_{\Delta}$ is constructed by induction at the level of the full blow up's, $\tilde{N}^{\text {all,* }}(K(\Delta))$ and $\widetilde{N}^{\text {all,*-1}}(\Delta)$, specifying its value on the elements $\mathbf{1}_{(F * G, \varepsilon)}$, with $F \triangleleft \Delta$ and $G \triangleleft \operatorname{Sub} \Delta$.

We set $\widetilde{\mathrm{T}}_{\Delta}\left(\mathbf{1}_{(F * G, \varepsilon)}\right)=0$ if $F * G$ is not full, in the sense of Definition 9.2 Now, let,

$$
\mathbf{1}_{(F * G, \varepsilon)}=\mathbf{1}_{\left(F_{0}, \varepsilon_{0}\right)} \otimes \cdots \otimes \mathbf{1}_{\left(F_{q} * G_{q}, \varepsilon_{q}\right)} \otimes \cdots \otimes \mathbf{1}_{\left(G_{n-1}, \varepsilon_{n-1}\right)} \otimes \mathbf{1}_{\left(G_{n}, \varepsilon_{n}\right)},
$$

with $F * G$ full. We use a double induction, on the length $n$ of the filtration of $\Delta$ and on the dimension of the component $\left(G_{n}, \varepsilon_{n}\right)$. We set $\Delta=\nabla * \Delta_{n}$.

a) If $\operatorname{dim}\left(G_{n}, \varepsilon_{n}\right)=0$, we distinguish three cases

i) If $G_{n}=\varnothing$ and $\varepsilon_{n}=1$, then $F * G \triangleleft \nabla$ and we set:

$$
\widetilde{\mathrm{T}}_{\Delta}\left(\mathbf{1}_{(F * G, \varepsilon)} \otimes \mathbf{1}_{(\varnothing, 1)}\right)=\widetilde{\mathrm{T}}_{\nabla}\left(\mathbf{1}_{(F * G, \varepsilon)}\right) \otimes \mathbf{1}_{(\varnothing, 1)} .
$$

ii) If $\operatorname{dim} G_{n}=0, \varepsilon_{n}=0$ and $q<n$, then $G_{n}=\left[e_{J}\right]$, where $e_{J}$ is the barycenter of a face of $\Delta$. Let $e_{\alpha}=\operatorname{der}\left(G_{q} * \cdots * G_{n}\right)$ the last vertex of the face $G$ and $G^{\prime}=G_{q} * \cdots * G_{n-1}$. (Notice $e_{\alpha} \in \Delta_{n}$, otherwise the weight of the vertex $e_{J}$ of $\operatorname{Sub} \Delta$ would be $n-1$.) We set:

$$
\widetilde{\mathrm{T}}_{\Delta}\left(\mathbf{1}_{\left(F * G^{\prime}, \varepsilon\right)} \otimes \mathbf{1}_{\left(\left[e_{J}\right], 0\right)}\right)=\widetilde{\mathrm{T}}_{\nabla}\left(\mathbf{1}_{\left(F * G^{\prime}, \varepsilon\right)}\right) \otimes \mathbf{1}_{\left(\left[e_{\alpha}\right], 0\right)} .
$$

iii) If $\operatorname{dim} G_{n}=0, \varepsilon_{n}=0$ and $q=n$, then $G=G_{n}=\left[e_{\{F\}}\right]$ is reduced to the barycenter of the face $F$. (Notice $F_{n} \neq \varnothing$ otherwise the weight of the barycenter of $F$ could not be $n$ in $\operatorname{Sub} \Delta$.) We set:

$$
\widetilde{\mathrm{T}}_{\Delta}\left(\mathbf{1}_{(F, \varepsilon)} * e_{\{F\}}\right)=(-1)^{|(F, \varepsilon)|} \mathbf{1}_{(F, \varepsilon)} .
$$

b) If $\operatorname{dim}\left(G_{n}, \varepsilon_{n}\right) \geqslant 1$, then $G_{n} \neq \varnothing$ and we set $e_{\alpha}=\operatorname{der}\left(G_{q} * \cdots * G_{n}\right)$ the last vertex of the face $G$. We write $G_{n}=G_{n}^{\prime} * e_{J}$, so that $G^{\prime}=G_{q} * \cdots * G_{n-1} * G_{n}^{\prime}$ is subdividing and $e_{\alpha}$ is a vertex of the face $J$ for which $e_{J}$ is the barycenter. We set:

$$
\widetilde{\mathrm{T}}_{\Delta}\left(\mathbf{1}_{\left(F * G^{\prime}, \varepsilon\right)} * e_{J}\right)=\widetilde{\mathrm{T}}_{\Delta}\left(\mathbf{1}_{\left(F * G^{\prime}, \varepsilon\right)}\right) * e_{\alpha} .
$$

The construction of $\widetilde{\mathrm{T}}_{\Delta}$ does not change the value of $\varepsilon_{n}$; moreover, the image of an element $\mathbf{1}_{(F * G, \varepsilon)}$ with $\varepsilon_{n}=0$ and $G_{n} \neq \varnothing$ also has a non-empty component in $\Delta_{n}$. So we built a linear map

$$
\widetilde{\mathrm{T}}_{\Delta}: \tilde{N}^{*}(K(\Delta)) \rightarrow \tilde{N}^{*-1}(\Delta) .
$$

We construct the map $\widetilde{\operatorname{Sub}_{\Delta}}: \widetilde{N}^{*}(\operatorname{Sub} \Delta) \rightarrow \widetilde{N}^{*}(\Delta)$ from $\widetilde{\mathrm{T}}_{\Delta}$, by

$$
\widetilde{\mathrm{Sub}}_{\Delta}=-\widetilde{\mathrm{T}}_{\Delta} \circ \widetilde{\delta}^{K(\Delta)} \text {. }
$$

REMARK 9.5. To any simplex, $F * G$, of $K(\Delta)$, we associate a family of vertices, $\mathcal{V}_{\Delta}(F * G)$, consisting of the vertices of $F$ and the vertices of the faces of $\Delta$ having a vertex of $G$ as barycenter. Adding the virtual vertices, we set $\mathcal{V}_{\Delta}\left(\mathbf{1}_{(F * G, \varepsilon)}\right)=$ $\mathcal{V}_{\Delta}(F * G, \varepsilon)$. By construction of $\widetilde{\mathrm{T}}_{\Delta}$, we have

$$
\mathcal{V}_{\Delta}\left(\mathbf{1}_{(F * G, \varepsilon)}\right)=\mathcal{V}_{\Delta}\left(\widetilde{\mathrm{T}}_{\Delta}\left(\mathbf{1}_{(F * G, \varepsilon)}\right)\right) \text {. }
$$

The proof of Lemma 9.4 is postponed to the end of the proof of Lemma 9.4 page 34. First, we take advantage of this result in terms of the blown-up intersection cohomology. 
Let $(X, \bar{p})$ be a perverse space. Recall that a cochain $\omega \in \tilde{N}_{\bar{p}}^{*}(X ; R)$ associates to any regular filtered simplex $\sigma: \Delta \rightarrow X$, a cochain $\omega_{\sigma} \in \tilde{N}_{\sigma}^{*}$ and verifies $\|\omega\| \leqslant \bar{p}$, $\|\delta \omega\| \leqslant \bar{p}$ and $\delta_{\ell}^{*}\left(\omega_{\sigma}\right)=\omega_{\partial_{\ell} \sigma}$, for any regular face operator, $\delta_{\ell}$.

Definition 9.6. Let $\mathcal{U}$ be an open cover of $X$. A $\mathcal{U}$-small simplex is a regular simplex, $\sigma: \Delta=\Delta_{0} * \cdots * \Delta_{n} \rightarrow X$, such that there exists $U \in \mathcal{U}$ with $\operatorname{Im} \sigma \subset U$. The family of $\mathcal{U}$-small simplices is denoted by $\operatorname{Simp}_{\mathcal{U}}$.

The blown-up complex of $\mathcal{U}$-small cochains of $X$ with coefficients in $R$, written $\tilde{N}^{*, \mathcal{U}}(X ; R)$, is the cochain complex made up of elements $\omega$, associating to any $\mathcal{U}$-small simplex, $\sigma: \Delta=\Delta_{0} * \cdots * \Delta_{n} \rightarrow X$, an element $\omega_{\sigma} \in \tilde{N}^{*}(\Delta)$, so that $\delta_{\ell}^{*}\left(\omega_{\sigma}\right)=\omega_{\partial_{\ell} \sigma}$, for any face operator, $\delta_{\ell}: \Delta_{0}^{\prime} * \cdots * \Delta_{n}^{\prime} \rightarrow \Delta_{0} * \cdots * \Delta_{n}$, with $\Delta_{n}^{\prime} \neq \varnothing$. If $\bar{p}$ is a perversity on $X$, we denote by $\tilde{N}_{\bar{p}}^{*, \mathcal{U}}(X ; R)$ the complex of $\mathcal{U}$-small cochains verifying $\|\omega\| \leqslant \bar{p}$ and $\|\delta \omega\| \leqslant \bar{p}$.

The following Theorem compares the complexes $\tilde{N}_{\bar{p}}^{*}(X ; R)$ and $\tilde{N}_{\bar{p}}^{*, \mathcal{U}}(X ; R)$.

TheOREm B. Let $(X, \bar{p})$ be a perverse space endowed with an open cover, $\mathcal{U}$. Let $\rho_{\mathcal{U}}: \tilde{N}_{\bar{p}}^{*}(X ; R) \rightarrow \tilde{N}_{\bar{p}}^{*, \mathcal{U}}(X ; R)$ be the restriction map. The following properties are verified.

(i) There exist a cochain map, $\varphi \mathcal{U}: \tilde{N}_{\bar{p}}^{*, \mathcal{U}}(X ; R) \rightarrow \tilde{N}_{\bar{p}}^{*}(X ; R)$, and a homotopy, $\Theta: \tilde{N}_{\bar{p}}^{*}(X ; R) \rightarrow \tilde{N}_{\bar{p}}^{*-1}(X ; R)$, such that

$$
\rho_{\mathcal{U}} \circ \varphi_{\mathcal{U}}=\mathrm{id} \text { and } \delta \circ \Theta+\Theta \circ \delta=\mathrm{id}-\varphi_{\mathcal{U}} \circ \rho_{\mathcal{U}} .
$$

(ii) Furthermore, if the cochain $\omega \in \tilde{N}_{\bar{p}}^{*, \mathcal{U}}(X ; R)$ is such that there exists a subset $K \subset X$ for which $\omega_{\sigma}=0$ if $(\operatorname{Im} \sigma) \cap K=\varnothing$, then $\varphi_{\mathcal{U}}(\omega)$ also verifies $\left(\varphi_{\mathcal{U}}(\omega)\right)_{\sigma}=0$ if $(\operatorname{Im} \sigma) \cap K=\varnothing$.

As an immediate consequence of property (i) we get the following corollary.

Corollary 9.7. The restriction map, $\rho_{\mathcal{U}}: \tilde{N}_{\bar{p}}^{*}(X ; R) \rightarrow \tilde{N}_{\bar{p}}^{*, \mathcal{U}}(X ; R)$, is a quasi-isomorphism.

First we establish the existence of a subdivision at the level of the blown-up complex.

Proposition 9.8. Consider $X$ a filtered space. The maps $\widetilde{\mathrm{T}}_{\Delta}$ and $\widetilde{\mathrm{Sub}} \Delta$ extend as $\widetilde{\mathrm{T}}: \tilde{N}^{*}(X ; R) \rightarrow \widetilde{N}^{*-1}(X ; R)$ and as $\widetilde{\mathrm{Sub}}: \widetilde{N}^{*}(X ; R) \rightarrow \widetilde{N}^{*}(X ; R)$ verifying

$$
\widetilde{\mathrm{T}} \circ \delta+\delta \circ \widetilde{\mathrm{T}}=\mathrm{id}-\widetilde{\mathrm{Sub}} \text {. }
$$

Proof. We first construct a homotopy, $\widetilde{\mathrm{T}}: \tilde{N}^{*}(X ; R) \rightarrow \tilde{N}^{*-1}(X ; R)$, from the maps $\widetilde{\mathrm{T}}_{\Delta}$ previously defined.

Let $\sigma: \Delta \rightarrow X$ be a filtered simplex and $\omega \in \tilde{N}^{k}(X ; R)$. A simplex $F * G$ of $K(\Delta)$ can be described by its vertices, $F * G=\left[e_{i_{0}}, \ldots, e_{i_{r}}, e_{\left\{F_{0}\right\}}, \ldots, e_{\left\{F_{s}\right\}}\right]$, where $e_{j}$ is a vertex of $\Delta$ and $e_{\left\{F_{j}\right\}} \in \mathcal{V}(\operatorname{Sub} \Delta)$ is the barycenter of the face $F_{j} \triangleleft \Delta$. It follows $e_{\left\{F_{j}\right\}} \in \Delta$ and we define a filtered simplex of $X, \sigma_{F * G}: F * G \rightarrow X$, using the barycentric coordinates by

$$
\sigma_{F * G}\left(\sum_{j} t_{j} e_{j}+\sum_{\ell} u_{\ell} e_{\left\{F_{\ell}\right\}}\right)=\sigma\left(\sum_{j} t_{j} e_{j}+\sum_{\ell} u_{\ell} e_{\left\{F_{\ell}\right\}}\right) .
$$


Thanks to Proposition 2.8, we define $\omega_{K(\Delta)} \in \tilde{N}^{k}(K(\Delta))$ by

$$
\omega_{K(\Delta)}=\sum_{\substack{F * G \triangleleft K(\Delta) \\|(F * G, \varepsilon)|=k}} \omega_{\sigma_{F * G}}(F * G, \varepsilon) \mathbf{1}_{(F * G, \varepsilon)} .
$$

Write now

$$
(\widetilde{\mathrm{T}}(\omega))_{\sigma}=\widetilde{\mathrm{T}}_{\Delta}\left(\omega_{K(\Delta)}\right)
$$

and verify that we obtain an element of $\tilde{N}^{*}(X ; R)$. Let $\sigma: \Delta=\Delta_{0} * \cdots * \Delta_{n} \rightarrow X$ be a regular simplex and $\tau=\sigma \circ \delta_{\ell}: \nabla \rightarrow X$, where $\delta_{\ell}: \nabla \rightarrow \Delta$ is a regular face operator. Since $\omega_{K(\Delta)} \in \tilde{N}^{k}(K(\Delta))$ then $\delta_{\ell}^{*} \omega_{K(\Delta)}=\omega_{K(\nabla)}$. The maps $\delta_{\ell}^{*}: \tilde{N}^{*}(\Delta) \rightarrow \tilde{N}^{*}(\nabla)$ and $\delta_{\ell}^{*}: \tilde{N}^{*}(K(\Delta)) \rightarrow \tilde{N}^{*}(K(\nabla))$ are defined by

$\delta_{\ell}^{*}\left(\mathbf{1}_{(F, \varepsilon)}\right)=\left\{\begin{array}{cl}\mathbf{1}_{(F, \varepsilon)} & \text { if } F \triangleleft \nabla, \\ 0 & \text { if not, }\end{array}\right.$ and $\delta_{\ell}^{*}\left(\mathbf{1}_{(F * G, \varepsilon)}\right)=\left\{\begin{array}{cl}\mathbf{1}_{(F * G, \varepsilon)} & \text { if } F * G \triangleleft K(\nabla), \\ 0 & \text { if not. }\end{array}\right.$

From these calculations and from Remark 9.5. directly follows the commutativity of the following diagram,

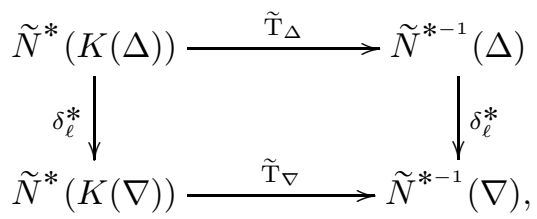

from which we deduce, $\delta_{\ell}^{*} \widetilde{\mathrm{T}}(\omega)_{\sigma}=\delta_{\ell}^{*} \widetilde{\mathrm{T}}_{\Delta}\left(\omega_{K(\Delta)}\right)=\widetilde{\mathrm{T}}_{\nabla}\left(\delta_{\ell}^{*} \omega_{K(\Delta)}\right)=\widetilde{\mathrm{T}}_{\nabla}\left(\omega_{K(\nabla)}\right)=$ $\widetilde{\mathrm{T}}(\omega)_{\tau}$. Thus the map $\widetilde{\mathrm{T}}: \tilde{N}^{*}(X ; R) \rightarrow \tilde{N}^{*-1}(X ; R)$ is well defined. Now we proceed to the construction of $\widetilde{\mathrm{Sub}}: \tilde{N}^{*}(X ; R) \rightarrow \tilde{N}^{*}(X ; R)$ from the operators $\widetilde{\mathrm{Sub}} \Delta$ of Lemma 9.4 Let $\sigma: \Delta \rightarrow X$ be a regular simplex and let $\omega \in \tilde{N}^{k}(X ; R)$. If $G$ is a face of Sub $\Delta$, we denote by $\iota_{G}: G \rightarrow \Delta$ the canonical injection and we define (cf. Proposition 2.8)

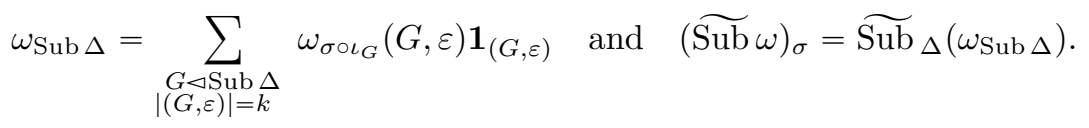

Notice

$$
\begin{aligned}
\iota_{\mathrm{Sub} \Delta}^{*}\left(\omega_{K(\Delta)}\right) & =\sum_{\substack{F * G \triangleleft K(\Delta) \\
|(F * G, \varepsilon)|=k}} \omega_{\sigma_{F * G}}(F * G, \varepsilon) \iota_{\mathrm{Sub} \Delta}^{*}\left(\mathbf{1}_{(F * G, \varepsilon)}\right) \\
& =\sum_{\substack{G \triangleleft \operatorname{Sub} \Delta \\
|(G, \varepsilon)|=k}} \omega_{\sigma \circ \iota_{G}}(G, \varepsilon) \mathbf{1}_{(G, \varepsilon)}=\omega_{\mathrm{Sub} \Delta} .
\end{aligned}
$$

We also have $\iota_{\Delta}^{*}\left(\omega_{K(\Delta)}\right)=\omega_{\sigma}$. It follows, by using Lemma 9.4

$$
\begin{aligned}
\omega_{\sigma}-(\tilde{\mathrm{T}} \delta \omega)_{\sigma}-(\delta \tilde{\mathrm{T}} \omega)_{\sigma} & =\iota_{\Delta}^{*}\left(\omega_{K(\Delta)}\right)-\widetilde{\mathrm{T}}_{\Delta}\left(\widetilde{\delta}^{K(\Delta)} \omega_{K(\Delta)}\right)-\widetilde{\delta}^{\Delta} \widetilde{\mathrm{T}}_{\Delta}\left(\omega_{K(\Delta)}\right) \\
& =\widetilde{\operatorname{Sub}} \Delta\left(\iota_{\operatorname{Sub} \Delta}^{*}\left(\omega_{K(\Delta)}\right)\right)=\widetilde{\operatorname{Sub}} \Delta\left(\omega_{\operatorname{Sub} \Delta}\right)=(\widetilde{\operatorname{Sub}}(\omega))_{\sigma}
\end{aligned}
$$

We have shown $\widetilde{\operatorname{Sub}}(\omega) \in \widetilde{N}^{*}(X ; R)$ and the equality $\widetilde{\mathrm{T}} \circ \delta+\delta \circ \widetilde{\mathrm{T}}=\mathrm{id}-\widetilde{\mathrm{Sub}}$. 
Proof of Theorem B, (i) We prove $\|\widetilde{\mathrm{T}}(\omega)\| \leqslant\|\omega\|$ and $\|\widetilde{\operatorname{Sub}}(\omega)\| \leqslant\|\omega\|$, by working on the chosen basis and the various possible cases. Making reference to Definition [2.5, since maps $\widetilde{\mathrm{T}}$ and $\widetilde{\mathrm{Sub}}$ do not modify the value of the parameter $\varepsilon$ then we can limit ourselves to the case $\varepsilon_{n-\ell}=0$, with $\ell \in\{1, \ldots, n\}$. We proceed by induction on the complex $\tilde{N}^{\text {all }}(\Delta)$, the case $n=0$ being obvious. With the notations used in the construction of $\widetilde{\mathrm{T}}$, the following properties are verified.

- (9.2): if $G_{n}=\varnothing$ and $\varepsilon_{n}=1$, the desired inequality comes from

$$
\begin{aligned}
\left\|\widetilde{\mathrm{T}}_{\Delta}\left(\mathbf{1}_{(F * G, \varepsilon)}\right) \otimes \mathbf{1}_{(\varnothing, 1)}\right\|_{\ell} & =\left\{\begin{array}{cc}
0 & \text { if } \ell=1, \\
\left\|\widetilde{\mathrm{T}}_{\Delta}\left(\mathbf{1}_{(F * G, \varepsilon)}\right)\right\|_{\ell-1} & \text { if } \ell>1,
\end{array}\right. \\
& \leqslant\left\{\begin{array}{cc}
0 & \text { if } \ell=1, \\
\left\|\mathbf{1}_{(F * G, \varepsilon)}\right\|_{\ell-1} & \text { if } \ell>1,
\end{array} \leqslant\left\|\mathbf{1}_{(F * G, \varepsilon)} \otimes \mathbf{1}_{(\varnothing, 1)}\right\|_{\ell .} .\right.
\end{aligned}
$$

- The justifications of (9.3) and (9.4) are similar.

- 9.5): if $\operatorname{dim}\left(G_{n}, \varepsilon_{n}\right) \geqslant 1$, we denote by $\ell(\alpha)$ the weight of the generator $e_{\alpha}$ of $\Delta$. By construction of $\widetilde{\mathrm{T}}_{\Delta}$, we have

$$
\left\|\widetilde{\mathrm{T}}_{\Delta}\left(\mathbf{1}_{\left(F * G^{\prime}, \varepsilon\right)}\right) * e_{\alpha}\right\|_{\ell}=\left\{\begin{array}{c}
\left\|\widetilde{\mathrm{T}}_{\Delta}\left(\mathbf{1}_{\left(F * G^{\prime}, \varepsilon\right)}\right)\right\|_{\ell \text { if } \ell(\alpha) \leqslant n-\ell,} \\
\left\|\widetilde{\mathrm{T}}_{\Delta}\left(\mathbf{1}_{\left(F * G^{\prime}, \varepsilon\right)}\right)\right\|_{\ell}+1 \text { if } \ell(\alpha)>n-\ell,
\end{array} \leqslant\left\|\mathbf{1}_{(F * G, \varepsilon)}\right\|_{\ell .}\right.
$$

To study the perverse degree of $\widetilde{\mathrm{Sub}_{\Delta}}(-)$, consider a subdividing face $G \triangleleft \operatorname{Sub} \Delta$ with first term $e_{0}$. By definition of $\widehat{\operatorname{Sub}} \Delta$, one has, for each $\ell \in\{1, \ldots, n\}$,

$\left\|\widetilde{\operatorname{Sub}}{ }_{\Delta} \mathbf{1}_{(G, \varepsilon)}\right\|_{\ell}=\left\|\widetilde{\mathrm{T}}_{\Delta} \widetilde{\delta}^{K(\Delta)} \mathbf{1}_{(G, \varepsilon)}\right\|_{\ell}=\left\|\widetilde{\mathrm{T}}_{\Delta}\left(\mathbf{1}_{(G, \varepsilon)} * e_{0}\right)\right\|_{\ell} \leqslant\left\|\mathbf{1}_{(G, \varepsilon)} * e_{0}\right\|_{\ell}=\left\|\mathbf{1}_{(G, \varepsilon)}\right\|_{\ell}$.

(The last equality uses the fact that $e_{0}$, being the first vertex of $G$, lies in the smallest filtration of $G$.) Together with Proposition 9.8, this proves that $\widetilde{\mathrm{T}}$ and $\widetilde{\mathrm{Sub}}$ define two maps from the complex $\tilde{N}_{\bar{p}}^{*}(X ; R)$ to itself.

We construct a cochain map, $\varphi_{\mathcal{U}}: \tilde{N}_{\bar{p}}^{*, \mathcal{U}}(X ; R) \rightarrow \tilde{N}_{\bar{p}}^{*}(X ; R)$, and a homotopy, $\Theta: \widetilde{N}_{\bar{p}}^{*}(X ; R) \rightarrow \widetilde{N}_{\bar{p}}^{*-1}(X ; R)$, such that $\rho_{\mathcal{U}} \circ \varphi_{\mathcal{U}}=\mathrm{id}$ and $\delta \circ \Theta+\Theta \circ \delta=\mathrm{id}-\varphi_{\mathcal{U}} \circ \rho_{\mathcal{U}}$.

Write $\Psi_{1}=\widetilde{\mathrm{T}}$ and, for $m \geqslant 2, \widetilde{\mathrm{Sub}}^{m}=\widetilde{\mathrm{Sub}}^{m-1} \circ \widetilde{\mathrm{Sub}}$ and $\Psi_{m}=\sum_{0 \leqslant i<m} \widetilde{\mathrm{T}} \circ$ $\widetilde{\mathrm{Sub}}^{i}$. A simple calculation using (9.6) gives

$$
\delta \circ \Psi_{m}+\Psi_{m} \circ \delta=\sum_{0 \leqslant i<m} \delta \circ \widetilde{\mathrm{T}} \circ \widetilde{\mathrm{Sub}}^{i}+\widetilde{\mathrm{T}}_{\widetilde{\mathrm{Sub}}^{i}} \circ \delta=\mathrm{id}-\widetilde{\mathrm{Sub}}^{m} .
$$

If $\sigma: \Delta \rightarrow X$ is a regular simplex, we denote by $m(\sigma)$ the smallest integer such that the simplices of $\mathrm{Sub}^{m(\sigma)} \Delta$ have their image by $\sigma$ included in an open subset of the cover $\mathcal{U}$. If $F$ is a simplex of $\Delta$, we denote by $\sigma_{F}: F \rightarrow X$ the restriction of $\sigma$ and by $(F, \varepsilon) \triangleleft \widetilde{\Delta}$ the fact that $(F, \varepsilon)$ is a face of the blow up $\widetilde{\Delta}$. Let $\omega \in \widetilde{N}^{k}(X ; R)$. The homotopy $\Theta$ is defined by

$$
(\Theta(\omega))_{\sigma}=\sum_{\substack{(F, \varepsilon) \triangleleft \widetilde{\Delta} \\|(F, \varepsilon)|=k-1}}\left(\Psi_{m\left(\sigma_{F}\right)}(\omega)\right)_{\sigma_{F}}(F, \varepsilon) \mathbf{1}_{(F, \varepsilon)} .
$$

Since the coefficient of $\mathbf{1}_{(F, \varepsilon)}$ depends only on $\sigma_{F}$ and $\varepsilon$ then we get the compatibility with the faces. In order to construct $\varphi_{\mathcal{U}}$, we calculate $\delta \circ \Theta$ and $\Theta \circ \delta$. Following 
(2.1), we have

$$
\delta \mathbf{1}_{(F, \varepsilon)}=(-1)^{k} \sum_{(F, \varepsilon) \triangleleft \partial(\nabla, \kappa)} n_{(F, \varepsilon, \nabla, \kappa)} \mathbf{1}_{(\nabla, \kappa)},
$$

where $(F, \varepsilon) \triangleleft \partial(\nabla, \kappa)$ means that $(F, \varepsilon)$ runs over the proper faces of $(\nabla, \kappa)$ and

$$
\partial(\nabla, \kappa)=\sum_{(F, \varepsilon) \triangleleft \partial(\nabla, \kappa)} n_{(F, \varepsilon, \nabla, \kappa)}(F, \varepsilon) .
$$

We deduce, for all $\omega \in \tilde{N}^{k}(X ; R)$,

$$
(\delta \Theta(\omega))_{\sigma}=(-1)^{k} \sum_{\substack{(F, \varepsilon) \triangleleft \widetilde{\Delta} \\|(F, \varepsilon)|=k-1}} \sum_{\substack{(F, \varepsilon) \triangleleft \partial(\nabla, \kappa) \\(\nabla, \kappa) \triangleleft \widetilde{\Delta}}} n_{(F, \varepsilon, \nabla, \kappa)}\left(\Psi_{m\left(\sigma_{F}\right)}(\omega)\right)_{\sigma_{F}}(F, \varepsilon) \mathbf{1}_{(\nabla, \kappa)} .
$$

On the other hand, the definition of $\Theta$ implies

$$
\begin{aligned}
& (\Theta(\delta \omega))_{\sigma}=\sum_{\substack{(\nabla, \kappa) \triangleleft \tilde{\Delta} \\
|(\nabla, \kappa)|=k}}\left(\Psi_{m\left(\sigma_{\nabla}\right)}(\delta \omega)\right)_{\sigma_{\nabla}}(\nabla, \kappa) \mathbf{1}_{(\nabla, \kappa)} \\
& =\sqrt{9.8} \omega_{\sigma}-\sum_{\substack{(\nabla, \kappa) \triangleleft \widetilde{\Delta} \\
|(\nabla, \kappa)|=k}}\left(\widetilde{\operatorname{Sub}}^{m\left(\sigma_{\nabla}\right)}(\omega)\right)_{\sigma_{\nabla}}(\nabla, \kappa) \mathbf{1}_{(\nabla, \kappa)} \\
& -\sum_{\substack{(\nabla, \kappa) \triangleleft \tilde{\Delta} \\
|(\nabla, \kappa)|=k}}\left(\delta \Psi_{m\left(\sigma_{\nabla}\right)}(\omega)\right)_{\sigma_{\nabla}}(\nabla, \kappa) \mathbf{1}_{(\nabla, \kappa)} .
\end{aligned}
$$

It follows, again by using (9.9),

$$
((\delta \Theta+\Theta \delta-\mathrm{id})(\omega))_{\sigma}=-\left(\varphi_{\mathcal{U}}(\omega)\right)_{\sigma}
$$

with

$$
\begin{array}{r}
-(\varphi \mathcal{U}(\omega))_{\sigma}=-\sum_{\substack{(\nabla, \kappa) \triangleleft \widetilde{\Delta} \\
|(\nabla, \kappa)|=k}}\left(\widetilde{\operatorname{Sub}}^{m\left(\sigma_{\nabla}\right)}(\omega)\right)_{\sigma_{\nabla}}(\nabla, \kappa) \mathbf{1}_{(\nabla, \kappa)}+ \\
\sum_{\substack{(F, \varepsilon) \triangleleft \widetilde{\Delta} \\
|(F, \varepsilon)|=k-1}} \sum_{\substack{(F, \varepsilon) \triangleleft \partial(\nabla, \kappa) \\
(\nabla, \kappa) \triangleleft \widetilde{\Delta}}} n_{(F, \varepsilon, \nabla, \kappa)}\left(\left(\Psi_{m\left(\sigma_{F}\right)}-\Psi_{m\left(\sigma_{\nabla}\right)}\right)(\omega)\right)_{\sigma_{F}}(F, \varepsilon) \mathbf{1}_{(\nabla, \kappa)} .
\end{array}
$$

Observe that $\left(\varphi_{\mathcal{U}}(\omega)\right)_{\sigma}$ is well defined for all $\omega \in \tilde{N}^{k}(X ; R)$ but we have to show that it is also well defined for every $\omega \in \widetilde{N}^{*, \mathcal{U}}(X ; R)$ and any regular simplex $\sigma: \Delta \rightarrow X$. The first term of the sum above is defined since the cochains $\left(\widetilde{\mathrm{Sub}}^{m}(\omega)\right)_{\sigma_{\nabla}}$ are well defined for any $m \geqslant m\left(\sigma_{\nabla}\right)$. The second term is also well defined since $m\left(\sigma_{F}\right) \leqslant$ $m\left(\sigma_{\nabla}\right)$ and the cochain $\left(\widetilde{\mathrm{Sub}}^{m}(\omega)\right)_{\sigma_{F}}$ is defined for any $m \geqslant m\left(\sigma_{F}\right)$. So we have $\varphi_{\mathcal{U}}(\omega) \in \tilde{N}^{*}(X ; R)$.

The equality $\delta \circ \Theta+\Theta \circ \delta=\mathrm{id}-\varphi_{\mathcal{U}} \circ \rho_{\mathcal{U}}$ is thus verified by construction of $\varphi_{\mathcal{U}}$ and $\Theta$. If $\omega \in \tilde{N}^{*, \mathcal{U}}(X ; R)$ and if $\sigma$ is $\mathcal{U}$-small, then $m(\sigma)=0$ and the family of indexes of the terms defining $\Psi_{m(\sigma)}$ is empty. Then

$$
\left(\rho_{\mathcal{U}}\left(\varphi_{\mathcal{U}}(\omega)\right)\right)_{\sigma}=\varphi_{\mathcal{U}}(\omega)_{\sigma}=\omega_{\sigma}-0=\omega_{\sigma} .
$$

We have therefore established $\rho_{\mathcal{U}} \circ \varphi_{\mathcal{U}}=$ id. From id $-\varphi_{\mathcal{U}}=\delta \circ \Theta+\Theta \circ \delta$, we deduce $\delta-\delta \circ \varphi_{\mathcal{U}}=\delta \circ \Theta \circ \delta=\delta-\varphi_{\mathcal{U}} \circ \delta$, and the compatibility of $\varphi_{\mathcal{U}}$ with differentials. 
It remains to study the behavior of $\Theta$ and $\varphi_{\mathcal{U}}$ relatively to the perverse degree. Since $\left\|\Psi_{m}(\omega)\right\| \leqslant\|\omega\|$ and $\|\widetilde{\operatorname{Sub}}(\omega)\| \leqslant\|\omega\|$, we have, for any singular stratum, $S,\|\Theta(\omega)\|_{S} \leqslant\|\omega\|_{S}$ and $\|\varphi \mathcal{U}(\omega)\|_{S} \leqslant\|\omega\|_{S}$. This completes the proof of the assertion (i).

(ii) Let $\omega \in \tilde{N}_{\bar{p}}^{*, \mathcal{U}}(X ; R)$ verifying the hypothesis of (ii) for a subset $K \subset X$. Let $\sigma: \Delta \rightarrow X$ such that $(\operatorname{Im} \sigma) \cap K=\varnothing$. The element $\left(\varphi_{\mathcal{U}}(\omega)\right)_{\sigma}$ is defined from cochains $\omega_{\sigma_{F * G}}$, where $\sigma_{F * G}$ is the restriction of $\sigma$, as defined in (9.7). From this formula, we find that $\operatorname{Im} \sigma_{F * G} \subset \operatorname{Im} \sigma$, and then $\omega_{\sigma_{F * G}}=0$ and $(\varphi \mathcal{U}(\omega))_{\sigma}=0$.

Proof of Lemma 9.4. We have to show that $\widetilde{\mathrm{T}}_{\Delta}: \tilde{N}^{*}(K(\Delta)) \rightarrow \tilde{N}^{*-1}(\Delta)$ verifies (9.1). For it, we use Proposition 2.11 to express the value of the differential $\delta$ in function of the adding of vertices.

(i) Start with $\mathbf{1}_{(F, \varepsilon)} \in \tilde{N}^{*}(\Delta)$. By definition, the face $F$ is not a full face (see Definition 9.2 on $K(\Delta)$, then we have $\widetilde{\mathrm{T}}_{\Delta}\left(\mathbf{1}_{(F, \varepsilon)}\right)=0$. On the other hand, the only term of $\widetilde{\delta}^{K(\Delta)} \mathbf{1}_{(F, \varepsilon)}$ having a non-zero image by $\widetilde{\mathrm{T}}_{\Delta}$ is $\mathbf{1}_{(F, \varepsilon)} * e_{\{F\}}$ where $e_{\{F\}}$ is the barycenter of the face $F$. Therefore one has, using (9.4),

$\widetilde{\mathrm{T}}_{\Delta}\left(\widetilde{\delta}^{K(\Delta)} \mathbf{1}_{(F, \varepsilon)}\right)=\widetilde{\mathrm{T}}_{\Delta}\left((-1)^{|(F, \varepsilon)|} \mathbf{1}_{(F, \varepsilon)} * e_{\{F\}}\right)=(-1)^{|(F, \varepsilon)|}(-1)^{|(F, \varepsilon)|} \mathbf{1}_{(F, \varepsilon)}=\mathbf{1}_{(F, \varepsilon)}$.

It follows:

$$
\left(\widetilde{\mathrm{T}}_{\Delta} \circ \widetilde{\delta}^{K(\Delta)}+\widetilde{\delta}^{\Delta} \circ \widetilde{\mathrm{T}}_{\Delta}\right)\left(\mathbf{1}_{(F, \varepsilon)}\right)=\left(\iota_{\Delta}^{*}-\widetilde{\operatorname{Sub}} \Delta \circ \iota_{\mathrm{Sub} \Delta}^{*}\right)\left(\mathbf{1}_{(F, \varepsilon)}\right) .
$$

(ii) Continue with a simplex, $F * G \triangleleft K(\Delta)$, such that $F \neq \varnothing$ and $G \neq \varnothing$. In this case, we have to show

$$
\left(\widetilde{\mathrm{T}}_{\Delta} \circ \widetilde{\delta}^{K(\Delta)}+\widetilde{\delta}^{\Delta} \circ \widetilde{\mathrm{T}}_{\Delta}\right)\left(\mathbf{1}_{(F * G, \varepsilon)}\right)=0 .
$$

- We look first to the case of a full simplex distinguishing the various possibilities that appear in the construction of $\widetilde{\mathrm{T}}_{\Delta}$, located after the statement of Lemma 9.4. We use a first recurrence assuming equality (9.10) holds for any filtered euclidean simplex with formal dimension strictly less than $n$.

- If $G_{n}=\varnothing$ and $\varepsilon_{n}=1$, we have $F * G \triangleleft K(\nabla)$, then:

$$
\begin{aligned}
& \widetilde{\mathrm{T}}_{\Delta}\left(\widetilde{\delta}^{K(\Delta)}\left(\mathbf{1}_{(F * G, \varepsilon)} \otimes \mathbf{1}_{(\varnothing, 1)}\right)\right)={ }_{(1)} \\
& \widetilde{\mathrm{T}}_{\nabla}\left(\widetilde{\delta}^{K(\nabla)} \mathbf{1}_{(F * G, \varepsilon)}\right) \otimes \mathbf{1}_{(\varnothing, 1)} \\
& +(-1)^{|(F * G, \varepsilon)|} \sum_{e \in \mathcal{V}\left(K(\Delta)_{n}\right)} \widetilde{\mathrm{T}}_{\Delta}\left(\mathbf{1}_{(F * G, \varepsilon)} \otimes \mathbf{1}_{(\varnothing, 1)} * e\right)=_{(2)} \\
& \widetilde{\mathrm{T}}_{\nabla}\left(\widetilde{\delta}^{K(\nabla)} \mathbf{1}_{(F * G, \varepsilon)} \otimes \mathbf{1}_{(\varnothing, 1)}\right. \\
& +(-1)^{|(F * G, \varepsilon)|} \sum_{e \in \mathcal{V}\left(\Delta_{n}\right)} \widetilde{\mathrm{T}}_{\nabla}\left(\mathbf{1}_{(F * G, \varepsilon)}\right) \otimes \mathbf{1}_{(\varnothing, 1)} * e={ }_{(3)} \\
& -\widetilde{\delta}^{\nabla} \widetilde{\mathrm{T}}_{\nabla}\left(\mathbf{1}_{(F * G, \varepsilon)} \otimes \mathbf{1}_{(\varnothing, 1)}+(-1)^{|(F * G, \varepsilon)|} \widetilde{\mathrm{T}}_{\nabla}\left(\mathbf{1}_{(F * G, \varepsilon)}\right) \otimes \widetilde{\delta}^{c \Delta_{n}} \mathbf{1}_{(\varnothing, 1)}={ }_{(1)}\right. \\
& \left.-\widetilde{\delta}^{\Delta} \widetilde{\mathrm{T}}_{\Delta}\left(\mathbf{1}_{(F * G, \varepsilon)} \otimes \mathbf{1}_{(\varnothing, 1)}\right)\right)
\end{aligned}
$$

where $={ }_{(1)}$ uses (9.2), $={ }_{(2)}$ uses $(9.5)$ and $(9.2),={ }_{(3)}$ is the induction hypothesis on $\nabla$.

- The argument is similar when $\operatorname{dim} G_{n}=0$ and $\varepsilon_{n}=0$. A second induction on the dimension of $G_{n}$ completes the proof in the case of a full simplex $F * G$. 
- If $F * G$ is not full and if the differential $\tilde{\delta}^{K(\Delta)} \mathbf{1}_{(F * G, \varepsilon)}$ only involves non full simplices, then the left hand side of (9.10) is zero and the result is true. So we have to consider the case of a non full simplex whose differential involves full simplices. Specifically, consider a full $k$-simplex $F^{\prime} * G^{\prime}$ with $F^{\prime}=\left[e_{i_{0}}, \ldots, e_{i_{a}}\right]$ and $G^{\prime}=\left[e_{\left\{i_{0} \ldots i_{a}\right\}}, \ldots, e_{\left\{i_{0} \ldots i_{a} \ldots i_{b}\right\}}\right]$ and including a non full $(k-1)$-face $F * G$ with $F \neq \varnothing$ and $G \neq \varnothing$.

We need to establish

$$
\widetilde{\mathrm{T}}_{\Delta} \widetilde{\delta}^{K(\Delta)}\left(\mathbf{1}_{(F * G, \varepsilon)}\right)=0 .
$$

Consider the various possible cases.

(a) Suppose $a=b$, then $F=\left[e_{i_{0}}, \ldots, \hat{e}_{i_{x}}, \ldots, e_{i_{a}}\right]$ and $G=G^{\prime}=\left[e_{\left\{F^{\prime}\right\}}\right]$. The equality (9.11) is reduced to $\widetilde{\mathrm{T}}_{\Delta} \widetilde{\delta}^{K(\Delta)}\left(\mathbf{1}_{(F, \varepsilon)} * e_{\left\{F^{\prime}\right\}}\right)=0$. By keeping in the expression of the differential only the elements corresponding to full simplices, we have:

$\widetilde{\mathrm{T}}_{\Delta} \widetilde{\delta}^{K(\Delta)}\left(\mathbf{1}_{(F, \varepsilon)} * e_{\left\{F^{\prime}\right\}}\right)=(-1)^{(F, \varepsilon)+1} \widetilde{\mathrm{T}}_{\Delta}\left(\mathbf{1}_{(F, \varepsilon)} * e_{\left\{F^{\prime}\right\}} * e_{i_{x}}+\mathbf{1}_{(F, \varepsilon)} * e_{\left\{F^{\prime}\right\}} * e_{\{F\}}\right)$

The definition of $\widetilde{\mathrm{T}}_{\Delta}$ gives,

$$
\begin{aligned}
\widetilde{\mathrm{T}}_{\Delta}\left(\mathbf{1}_{(F, \varepsilon)} * e_{\left\{F^{\prime}\right\}} * e_{i_{x}}\right)= & -\widetilde{\mathrm{T}}_{\Delta}\left(\mathbf{1}_{(F, \varepsilon)} * e_{i_{x}} * e_{\left\{F^{\prime}\right\}}\right)=-(-1)^{\left|\left(F^{\prime}, \varepsilon\right)\right|} \mathbf{1}_{(F, \varepsilon)} * e_{i_{x}}, \\
& \widetilde{\mathrm{T}}_{\Delta}\left(\mathbf{1}_{(F, \varepsilon)} * e_{\left\{F^{\prime}\right\}} * e_{\{F\}}\right)=-\widetilde{\mathrm{T}}_{\Delta}\left(\mathbf{1}_{(F, \varepsilon)} * e_{\{F\}} * e_{\left\{F^{\prime}\right\}}\right) \\
& =-\widetilde{\mathrm{T}}_{\Delta}\left(\mathbf{1}_{(F, \varepsilon)} * e_{\{F\}}\right) * e_{i_{x}}=-(-1)^{|(F, \varepsilon)|} \mathbf{1}_{(F, \varepsilon)} * e_{i_{x}} .
\end{aligned}
$$

The conclusion comes from $|(F, \varepsilon)|+1=\left|\left(F^{\prime}, \varepsilon\right)\right|$.

For the last two cases, we use an induction on the dimension of the component $G_{n}$.

(b) Suppose $a<b$ and $F=\left[e_{i_{0}}, \ldots, \hat{e}_{i_{x}}, \ldots, e_{i_{a}}\right]$, then $G=G^{\prime}=L * e_{\left\{i_{0} \ldots i_{a} \ldots i_{b}\right\}}$. The equality (9.11) is reduced to $\widetilde{\mathrm{T}}_{\Delta} \widetilde{\delta}^{K(\Delta)}\left(\mathbf{1}_{(F * L, \varepsilon)} * e_{\left\{i_{0} \ldots i_{a} \ldots i_{b}\right\}}\right)=0$. By definition of $\widetilde{\mathrm{T}}_{\Delta}$ and following Corollary 2.12, one has:

$$
\widetilde{\mathrm{T}}_{\Delta} \widetilde{\delta}^{K(\Delta)}\left(\mathbf{1}_{(F * L, \varepsilon)} * e_{\left\{i_{0} \ldots i_{a} \ldots i_{b}\right\}}\right)=\widetilde{\mathrm{T}}_{\Delta}\left(\widetilde{\delta}^{K(\Delta)} \mathbf{1}_{(F * L, \varepsilon)}\right) * e_{i_{b}} .
$$

The simplex $F * L$ is not full and $L \neq \varnothing$ since $a<b$. We can apply the induction hypothesis, so $\widetilde{\mathrm{T}}_{\Delta} \widetilde{\delta}^{K(\Delta)}\left(\mathbf{1}_{(F * L, \varepsilon)}\right)=0$.

(c) The argument is similar when $G=\left[e_{\left\{i_{0} \ldots i_{a}\right\}}, \ldots, \hat{e}_{\left\{i_{0} \ldots i_{a} \ldots i_{x}\right\}}, \ldots, e_{\left\{i_{0} \ldots i_{a} \ldots i_{b}\right\}}\right]$ and $a<b$, and thus $F=F^{\prime}$.

(iii) It remains to verify the equality (9.1) for elements $\mathbf{1}_{(G, \varepsilon)} \in \tilde{N}^{*}(\operatorname{Sub} \Delta)$. In this case, it is actually the definition of $\widetilde{\mathrm{Sub}}$ by

$$
\widetilde{\operatorname{Sub}} \mathbf{1}_{(G, \varepsilon)}=-\left(\widetilde{\mathrm{T}}_{\Delta} \circ \widetilde{\delta}^{K(\Delta)}\right)\left(\mathbf{1}_{(G, \varepsilon)}\right),
$$

the other terms being zero.

To complete the proof of Lemma 9.4, it remains to verify the compatibility of $\widetilde{\mathrm{Sub}}$ with differentials,

$$
\tilde{\delta}^{\Delta} \circ \widetilde{\mathrm{T}}_{\Delta} \circ \tilde{\delta}^{K(\Delta)}\left(\mathbf{1}_{(G, \varepsilon)}\right)=\widetilde{\mathrm{T}}_{\Delta} \circ \tilde{\delta}^{K(\Delta)} \circ \widetilde{\delta}^{\mathrm{Sub} \Delta}\left(\mathbf{1}_{(G, \varepsilon)}\right) .
$$

The two terms of this equation are zero except in the two following situations.

(i) $G=\left[e_{i_{0}}, \ldots, e_{\left\{i_{0} \ldots i_{a}\right\}}\right]$,

(ii) $G=\left[e_{i_{0}}, \ldots, \hat{e}_{\left\{i_{0} \ldots i_{x}\right\}}, \ldots, e_{\left\{i_{0} \ldots i_{x} \ldots i_{a}\right\}}\right]$ with $x<a$. 
Detail each case.

(i) The term $\tilde{\delta}^{K(\Delta)}\left(\mathbf{1}_{(G, \varepsilon)}\right)$ has only one full term, $(-1)^{|(G, \varepsilon)|} \mathbf{1}_{(G, \varepsilon)} * e_{i_{0}}$. The term $\widetilde{\delta}^{K(\Delta)} \widetilde{\delta}^{\operatorname{Sub} \Delta}\left(\mathbf{1}_{(G, \varepsilon)}\right)$ has the following full terms $-\sum_{e_{i_{j}} \in \mathcal{V}(\Delta)} \mathbf{1}_{(G, \varepsilon)} * e_{\left\{i_{0} \ldots i_{a} i_{j}\right\}} *$ $e_{i_{0}}$. We can deduce,

$$
\begin{aligned}
\widetilde{\mathrm{T}}_{\Delta} \widetilde{\delta}^{K(\Delta)} \tilde{\delta}^{\operatorname{Sub} \Delta}\left(\mathbf{1}_{(G, \varepsilon)}\right) & =-\sum_{e_{i_{j}} \in \mathcal{V}(\Delta)} \widetilde{\mathrm{T}}_{\Delta}\left(\mathbf{1}_{(G, \varepsilon)} * e_{\left\{i_{0} \ldots i_{a} i_{j}\right\}} * e_{i_{0}}\right) \\
& =\sum_{e_{i_{j}} \in \mathcal{V}(\Delta)} \widetilde{\mathrm{T}}_{\Delta}\left(\mathbf{1}_{(G, \varepsilon)} * e_{i_{0}}\right) * e_{i_{j}} \\
& =(-1)^{|(G, \varepsilon)|} \tilde{\delta}^{\Delta} \widetilde{\mathrm{T}}_{\Delta}\left(\mathbf{1}_{(G, \varepsilon)} * e_{i_{0}}\right)=\widetilde{\delta}^{\Delta} \widetilde{\mathrm{T}}_{\Delta} \tilde{\delta}^{K(\Delta)}\left(\mathbf{1}_{(G, \varepsilon)}\right) .
\end{aligned}
$$

(ii) The term $\tilde{\delta}^{K(\Delta)} \mathbf{1}_{(G, \varepsilon)}$ does not have any full term. Also, the next term $\widetilde{\delta}^{K(\Delta)} \tilde{\delta}^{\operatorname{Sub} \Delta} \mathbf{1}_{(G, \varepsilon)}$ has the following full terms $-\mathbf{1}_{(G, \varepsilon)} * e_{\left\{i_{0} \ldots i_{x}\right\}} * e_{i_{0}}-\mathbf{1}_{(G, \varepsilon)} *$ $e_{\left\{i_{0} \ldots i_{x-1} i_{x+1}\right\}} * e_{i_{0}}$. As in the previous calculation, equation (9.12) follows from the definition of $\widetilde{\mathrm{T}}_{\Delta}$ :

$$
\widetilde{\mathrm{T}}_{\Delta}\left(\mathbf{1}_{(G, \varepsilon)} * e_{\left\{i_{0} \ldots i_{x}\right\}} * e_{i_{0}}\right)=-\widetilde{\mathrm{T}}_{\Delta}\left(\mathbf{1}_{(G, \varepsilon)} * e_{\left\{i_{0} \ldots i_{x-1} i_{x+1}\right\}} * e_{i_{0}}\right) .
$$

(The sign comes from the permutation of $e_{i_{x}}$ and $e_{i_{x+1}}$.)

\section{Mayer-Vietoris exact sequence. Theorem C.}

If $U \subset \mathrm{V}$ are two open subsets of a perverse space $(X, \bar{p})$, canonical inclusions $U \subset V \subset X$ induce cochain maps, $\widetilde{N}_{\bar{p}}^{*}(X ; R) \rightarrow \widetilde{N}_{\bar{p}}^{*}(V ; R) \rightarrow \widetilde{N}_{\bar{p}}^{*}(U ; R)$. If there is no ambiguity, we keep the same notation for a cochain and its images by these maps.

Theorem C (Mayer-Vietoris exact sequence). Let $(X, \bar{p})$ be a paracompact perverse space, endowed with an open cover $\left(U_{1}, U_{2}\right)$ and a subordinated partition of the unity, $\left(f_{1}, f_{2}\right)$. For $i=1,2$, we denote by $\mathcal{U}_{i}$ the cover of $U_{i}$ consisting of the open subsets $\left.\left(U_{1} \cap U_{2}, f_{i}^{-1}(] 1 / 2,1\right]\right)$ and by $\mathcal{U}$ the cover of $X$, union of the covers $\mathcal{U}_{i}$. Then, the canonical inclusions, $U_{i} \subset X$ and $U_{1} \cap U_{2} \subset U_{i}$, induce a short exact sequence,

$$
0 \rightarrow \tilde{N}_{\bar{p}}^{*, \mathcal{U}}(X ; R) \stackrel{\iota}{\longrightarrow} \tilde{N}_{\bar{p}}^{*, \mathcal{U}_{1}}\left(U_{1} ; R\right) \oplus \tilde{N}_{\bar{p}}^{*, \mathcal{U}_{2}}\left(U_{2} ; R\right) \stackrel{\varphi}{\longrightarrow} \tilde{N}_{\bar{p}}^{*}\left(U_{1} \cap U_{2} ; R\right) \rightarrow 0,
$$

where $\varphi\left(\omega_{1}, \omega_{2}\right)=\omega_{1}-\omega_{2}$.

The following result is a direct consequence of Theorems $\mathrm{B}$ and $\mathrm{C}$.

COROLlaRY 10.1. Let $(X, \bar{p})$ be a paracompact perverse space provided with an open cover $\left(U_{1}, U_{2}\right)$. Then there is a long exact sequence for the blown-up intersection cohomology,

$$
\rightarrow \mathscr{H}_{\bar{p}}^{i}(X ; R) \rightarrow \mathscr{H}_{\bar{p}}^{i}\left(U_{1} ; R\right) \oplus \mathscr{H}_{\bar{p}}^{i}\left(U_{2} ; R\right) \rightarrow \mathscr{H}_{\bar{p}}^{i}\left(U_{1} \cap U_{2} ; R\right) \rightarrow \mathscr{H}_{\bar{p}}^{i+1}(X ; R) \rightarrow
$$

Proof of Theorem C. Since the open cover $\mathcal{U}$ is the union of the covers $\mathcal{U}_{1}$ and $\mathcal{U}_{2}$, the morphism $\iota$ is injective.

We prove the surjectivity of the map $\varphi$. For $i=1,2$, we define the fonction, $g_{i}: X \rightarrow\{0,1\}$, by

$$
g_{1}(x)=\left\{\begin{array}{ll}
1 & \text { if } f_{1}(x)>1 / 2, \\
0 & \text { if not },
\end{array} \quad \text { and } \quad g_{2}(x)= \begin{cases}1 & \text { if } f_{2}(x) \geqslant 1 / 2 \\
0 & \text { if not. }\end{cases}\right.
$$


The inequality $f_{1}(x)>1 / 2$ implies $f_{2}(x)<1 / 2$ and $g_{2}(x)=0$. The support of $g_{2}$ is therefore included in $\left.\left.U_{2} \backslash f_{1}^{-1}(] 1 / 2,1\right]\right)$. It is noted as well that the support of $g_{1}$ is included in $\left.\left.U_{1} \backslash f_{2}^{-1}(] 1 / 2,1\right]\right)$. On the other hand, by construction, one has $g_{1}(x)+g_{2}(x)=1$. We denote by $\tilde{g}_{1}$ and $\tilde{g}_{2}$ the two 0 -cochains of perverse degree 0 , respectively associated to $g_{1}$ and $g_{2}$, and defined in Lemma 10.2, If $\omega \in \tilde{N}_{\bar{p}}^{*}\left(U_{1} \cap U_{2} ; R\right)$, we denote by $\tilde{g}_{i} \cup \omega$ the cup product (cf. Section 4 ) of $\tilde{g}_{i}$ with $\omega$, for $i=1,2$. Since the cochain $\tilde{g}_{1}$ has a support included in $\left.\left.U_{1} \backslash f_{2}^{-1}(] 1 / 2,1\right]\right)$, then the cup product $\tilde{g}_{1} \cup \omega \in \tilde{N}_{\bar{p}}^{*, \mathcal{u}_{1}}\left(U_{1} ; R\right)$. Likewise, one has $\tilde{g}_{2} \cup \omega \in \widetilde{N}_{\bar{p}}^{*, \mathcal{u}_{2}}\left(U_{2} ; R\right)$. We verify $\varphi\left(\tilde{g}_{1} \cup \omega,-\tilde{g}_{2} \cup \omega\right)=\omega$, which gives the surjectivity of $\varphi$.

The composition $\varphi \circ \iota$ is the zero map. It remains to consider an element $\left(\omega_{1}, \omega_{2}\right) \in \widetilde{N}_{\bar{p}}^{*, \mathcal{U}_{1}}\left(U_{1} ; R\right) \oplus \widetilde{N}_{\bar{p}}^{*, \mathcal{U}_{2}}\left(U_{2} ; R\right)$ such that $\varphi\left(\omega_{1}, \omega_{2}\right)=0$ and to construct $\omega \in \widetilde{N}_{\bar{p}}^{*, \mathcal{U}}(X ; R)$ such that $\iota(\omega)=\left(\omega_{1}, \omega_{2}\right)$. If $\sigma: \Delta \rightarrow X$ is a regular simplex, we set,

$$
\begin{aligned}
& \text { - } \omega_{\sigma}=\left(\omega_{1}\right)_{\sigma}=\left(\omega_{2}\right)_{\sigma}, \text { if } \sigma(\Delta) \subset U_{1} \cap U_{2}, \\
& \text { - } \left.\left.\omega_{\sigma}=\left(\omega_{1}\right)_{\sigma}, \text { if } \sigma(\Delta) \subset f_{1}^{-1}(] 1 / 2,1\right]\right), \\
& \text { - } \left.\left.\omega_{\sigma}=\left(\omega_{2}\right)_{\sigma}, \text { if } \sigma(\Delta) \subset f_{2}^{-1}(] 1 / 2,1\right]\right) .
\end{aligned}
$$

This definition makes sense because, on the one hand $\left.\left.\left.\left.f_{1}^{-1}(] 1 / 2,1\right]\right) \cap f_{2}^{-1}(] 1 / 2,1\right]\right)=$ $\varnothing$ and on the other hand $\left.\left.U_{1} \cap U_{2} \cap f_{1}^{-1}(] 1 / 2,1\right]\right) \subset U_{1} \cap U_{2}$, where the two cochains $\left(\omega_{1}\right)_{\sigma}$ and $\left(\omega_{2}\right)_{\sigma}$ coincide.

With the notation of the previous proof, the connecting morphism of the long exact sequence of Mayer-Vietoris is defined by

$$
[\omega] \mapsto\left[\left(\delta \tilde{g}_{1}\right) \cup \omega\right] .
$$

Lemma 10.2. Let $(X, \bar{p})$ be a perverse space. Any map, $g: X \rightarrow R$, defines a 0-cochain $\tilde{g} \in \tilde{N}_{\overline{0}}^{0}(X ; R)$. Moreover, the association $g \mapsto \tilde{g}$ is $R$-linear.

Proof. Let $\sigma: \Delta=\Delta_{0} * \cdots * \Delta_{n} \rightarrow X$ be a regular simplex. We want to define $\tilde{g}_{\sigma} \in N^{0}\left(c \Delta_{0}\right) \otimes \cdots \otimes N^{0}\left(c \Delta_{n-1}\right) \otimes N^{0}\left(\Delta_{n}\right)$. If $b=\left(b_{0}, \ldots, b_{n}\right) \in c \Delta_{0} \times \cdots \times$ $c \Delta_{n-1} \times \Delta_{n}$, we denote by $i_{0}$ the smallest index for which $b_{i}$ is not a apex of a cone (i.e., $i_{0}=\min \left\{i \mid b_{i} \in \Delta_{i}\right\}$ ). Observe that the integer $i_{0}$ exists since $b_{n} \in \Delta_{n}$ and set $\tilde{g}_{\sigma}(b)=g\left(\sigma\left(b_{i_{0}}\right)\right)$. This map is clearly compatible with the face operators and defines $\tilde{g} \in \widetilde{N}^{0}(X ; R)$.

It remains to determine the perversity of the cochain $\tilde{g}_{\sigma}$. Since it is a 0 -cochain, it is obviously $\overline{0}$-allowable and we only need to study its coboundary. To compute the $\ell$-perverse degree of $\delta \tilde{g}_{\sigma}$, we consider $F=F_{0} \otimes \cdots \otimes F_{n}=\left\{b_{0}\right\} \otimes \cdots \otimes F_{j} \otimes \cdots \otimes\left\{b_{n}\right\}$ with $\operatorname{dim} F_{j}=1$ and $\partial F_{j}=b_{j}^{1}-b_{j}^{0}$. We have,

$$
\delta \tilde{g}_{\sigma}(F)=\tilde{g}_{\sigma}\left(b_{0}, \ldots, b_{j}^{1}, \ldots, b_{n}\right)-\tilde{g}_{\sigma}\left(b_{0}, \ldots, b_{j}^{0}, \ldots, b_{n}\right) .
$$

If $n-\ell<j$, then $i_{0} \leqslant n-\ell<j$ and $\delta \tilde{g}_{\sigma}(F)=g\left(\sigma\left(b_{i_{0}}\right)\right)-g\left(\sigma\left(b_{i_{0}}\right)\right)=0$, and then $\left\|\delta \tilde{g}_{\sigma}\right\|_{\ell}=-\infty$.

Finally, $\tilde{g} \in \tilde{N}_{\bar{\sigma}}^{0}(X)$ and the association $g \mapsto \tilde{g}$ is $R$-linear by construction.

\section{Product with the real line. Theorem $\mathrm{D}$.}

Consider the product $X \times \mathbb{R}$ equipped with the product filtration $(X \times \mathbb{R})_{i}=$ $X_{i} \times \mathbb{R}$ and a perversity $\bar{p}$. We also denote by $\bar{p}$ the perversity induced on $X$, that is, $\bar{p}(S)=\bar{p}(S \times \mathbb{R})$ for each stratum $S$ of $X$. Let $I_{0} I_{1}: X \rightarrow X \times \mathbb{R}$ be the canonical injections, defined by $I_{0}(x)=(x, 0)$ and $I_{1}(x)=(x, 1)$. The canonical projections 
are denoted by pr: $X \times \mathbb{R} \rightarrow X$ and $\operatorname{pr}_{2}: X \times \mathbb{R} \rightarrow \mathbb{R}$. Proposition 3.5 ensures that the maps $I_{0}, I_{1}$ and pr induce cochain maps between the blown-up complexes.

Theorem D. Let $X$ be a filtered space and let $X \times \mathbb{R}$ equipped with the filtration product and a perversity $\bar{p}$. Denoting also $\bar{p}$ the perversity induced on $X$, the maps induced in the blown-up intersection cohomology by the projection $\operatorname{pr}: X \times \mathbb{R} \rightarrow X$ and the canonical injections, $I_{0} I_{1}: X \rightarrow X \times \mathbb{R}$, verify $\left(I_{0} \circ \mathrm{pr}\right)^{*}=\left(I_{1} \circ \mathrm{pr}\right)^{*}=\mathrm{id}$.

The proof proceeds according to the scheme of Section 9 by constructing a homotopy $\Theta_{\Delta}$ at the simplex level (cf. Proposition 11.1), then gluing them to get a homotopy at the level of blown-up complexes (see Proposition 11.3).

For any filtered simplex, $\Delta=\Delta_{0} * \cdots * \Delta_{n}$, one defines a weighted simplicial complex $\mathcal{L}_{\Delta}=\Delta \otimes[0,1]$, whose simplices are the joins $(F, \mathbf{0}) *(G, \mathbf{1})$, with $F \triangleleft \Delta$, $G \triangleleft \Delta,(F, \mathbf{0}) \subset \Delta \times\{0\}$ and $(G, \mathbf{1}) \subset \Delta \times\{1\}$. Henceforth, we denote by $F * G$ such simplex, meaning that the first term, $F$, is identified with a simplex of $\Delta \times\{0\}$ and the second, $G$, with a simplex of $\Delta \times\{1\}$.

If $F$ and $G$ are compatible, in the sense of Definition 4.1 then, for the filtration induced by $\Delta$, we have $F * G=F_{0} * \cdots *\left(F_{p} * G_{p}\right) * \cdots * G_{n}$, with $p \in\{0, \ldots, n\}$. A face of the prismatic set $\widetilde{\mathcal{L}}_{\Delta}$, corresponding to compatible simplices, $F$ and $G$ of $\Delta$, is denoted by,

$$
(F * G, \varepsilon)=\left(F_{0}, \varepsilon_{0}\right) \times \cdots \times\left(F_{p} * G_{p}, \varepsilon_{p}\right) \times \cdots \times\left(G_{n-1}, \varepsilon_{n-1}\right) \times G_{n} .
$$

In this writing, if $j<n, j \neq p$, one authorizes $F_{j}$ and $G_{j}$ to be the emptyset but the associated variable $\varepsilon_{j}$ must be 1 .

Proposition 11.1. There exists a linear map, $\Theta_{\Delta}: \widetilde{N}^{*}(\Delta \otimes[0,1]) \rightarrow \widetilde{N}^{*-1}(\Delta)$, such that

$$
\left(\Theta_{\Delta} \circ \delta+\delta \circ \Theta_{\Delta}\right)\left(\mathbf{1}_{(F * G, \varepsilon)}\right)=\left\{\begin{array}{cl}
0 & \text { if } F \neq \varnothing \text { and } G \neq \varnothing \\
-\mathbf{1}_{(G, \varepsilon)} & \text { if } F=\varnothing \\
\mathbf{1}_{(F, \varepsilon)} & \text { if } G=\varnothing
\end{array}\right.
$$

where $\delta$ denotes the differential of $\widetilde{N}^{*}(\Delta \otimes[0,1])$ and $\widetilde{N}^{*-1}(\Delta)$.

Proof. Define the map $\Theta_{\Delta}$ on the elements of the dual basis. Let

$$
\mathbf{1}_{(F * G, \varepsilon)}=\mathbf{1}_{\left(F_{0}, \varepsilon_{0}\right)} \otimes \cdots \otimes \mathbf{1}_{\left(F_{p} * G_{p}, \varepsilon_{p}\right)} \otimes \cdots \otimes \mathbf{1}_{\left(G_{n-1}, \varepsilon_{n-1}\right)} \otimes \mathbf{1}_{G_{n}} .
$$

We set

$$
\Theta_{\Delta}\left(\mathbf{1}_{(F * G, \varepsilon)}\right)=\left\{\begin{array}{cl}
(-1)^{|(F, \varepsilon)|_{<p}+\left|\left(G_{p}, \varepsilon_{p}\right)\right|} \mathbf{1}_{(F \cup G, \varepsilon)} & \text { if } F \text { and } G \text { are compatible, } \\
0 & \text { if not, }
\end{array}\right.
$$

where $\mathbf{1}_{(F \cup G, \varepsilon)}=\mathbf{1}_{\left(F_{0}, \varepsilon_{0}\right)} \otimes \cdots \otimes \mathbf{1}_{\left(F_{p} \cup G_{p}, \varepsilon_{p}\right)} \otimes \cdots \otimes \mathbf{1}_{\left(G_{n-1}, \varepsilon_{n-1}\right)} \otimes \mathbf{1}_{G_{n}}$ and $F_{p} \cup G_{p}$ was introduced in Definition 4.1 .

In $\mathbf{1}_{(F * G, \varepsilon)}$, we consider the faces $F$ of $\Delta \times\{0\}$ and $G$ of $\Delta \times\{1\}$. In the expression of $\Theta_{\Delta}\left(\mathbf{1}_{(F * G, \varepsilon)}\right)$, we make an abuse of notation by keeping the letters $F$ and $G$ for faces of $\Delta$. It remains to verify (11.1) and for that, we consider the following cases. We denote by $\mathcal{V}\left(\tilde{\mathcal{L}}_{\Delta}\right)$ the union of $\mathcal{V}\left(\mathcal{L}_{\Delta}\right)$ with the family of virtual vertices. 
- Suppose $F \neq \varnothing, G \neq \varnothing, F$ and $G$ compatible. Using equality (2.6) and Lemma 11.2, we obtain the equality (11.1) in this case:

$$
\begin{aligned}
\delta \Theta_{\Delta}\left(\mathbf{1}_{(F * G, \varepsilon)}\right) & =(-1)^{|(F * G, \varepsilon)|+1} \sum_{e \in \mathcal{V}(\widetilde{\Delta})} \Theta_{\Delta}\left(\mathbf{1}_{(F * G, \varepsilon)}\right) * e \\
& =(-1)^{|(F * G, \varepsilon)|+1} \sum_{e \in \mathcal{V}\left(\widetilde{\mathcal{L}}_{\Delta}\right)} \Theta_{\Delta}\left(\mathbf{1}_{(F * G, \varepsilon)} * e\right)=-\Theta_{\Delta}\left(\delta \mathbf{1}_{(F * G, \varepsilon)}\right) .
\end{aligned}
$$

- Suppose $F \neq \varnothing, G \neq \varnothing, F$ and $G$ not compatible such that $\delta \mathbf{1}_{(F * G, \varepsilon)}$ contains compatible elements. This amounts to giving a $k$-simplex, $F^{\prime} * G^{\prime}$, of $\Delta \otimes[0,1]$, with $F^{\prime}$ and $G^{\prime}$ compatible and having a $(k-1)$-face, $F * G$, with $F$ and $G$ non compatible and non empty. Put $F^{\prime}=\left[a_{i_{0}}, \ldots, a_{i_{r}}\right]$ and $G^{\prime}=\left[b_{j_{0}}, \ldots, b_{j_{s}}\right]$ with $a_{i_{r}}=b_{j_{0}} \in \Delta_{p}$. (Recall that in the latter equality we identified $\Delta \cong \Delta \times\{0\} \cong \Delta \times\{1\}$.) We denote by $a_{j_{t}} \in \Delta \times\{0\}$ the vertex identified to $b_{j_{t}} \in \Delta \times\{1\}$, as well as $b_{i_{t}} \in \Delta \times\{1\}$ for the vertex identified to $a_{i_{t}} \in \Delta \times\{0\}$. For the simplices $F$ and $G$, there are only two possibilities corresponding to compatible simplices $F^{\prime}, G^{\prime}$. Equality (11.1) is deduced from the following calculations.

+ If $F=\left[a_{i_{0}}, \ldots, a_{i_{r-1}}\right]$ and $G=G^{\prime}$, the expression $\Theta_{\Delta}\left(\delta \mathbf{1}_{(F * G, \varepsilon)}\right)$ takes the values,

$$
(-1)^{|(G, \varepsilon)|+1+|(F, \varepsilon)|_{<p}+\varepsilon_{p}} \Theta_{\Delta}\left(\mathbf{1}_{\left(F * a_{i_{r}} * G, \varepsilon\right)}+\mathbf{1}_{\left(F * b_{i_{r-1}} * G, \varepsilon\right)}\right),
$$

if $a_{i_{r-1}} \in F_{p}$, and

$$
(-1)^{|(F, \varepsilon)|+\mid\left(G_{p}, \varepsilon_{p}\right)+1} \Theta_{\Delta}\left(\mathbf{1}_{\left(F * a_{i_{r}} * G, \varepsilon\right)}\right)+(-1)^{|(F, \varepsilon)|_{<a}+\varepsilon_{a}} \Theta_{\Delta}\left(\mathbf{1}_{\left(F * b_{i_{r-1}} * G, \varepsilon\right)}\right),
$$

if $a_{i_{r}-1} \in F_{a}$ with $a<p$, which implies

$$
\Theta_{\Delta}\left(\delta \mathbf{1}_{(F * G, \varepsilon)}\right)=-\mathbf{1}_{\left(\left(F * a_{i_{r}}\right) \cup G, \varepsilon\right)}+\mathbf{1}_{\left(F \cup\left(b_{i_{r-1}} * G\right), \varepsilon\right)}=0 .
$$

+ The argument is the same for $F=F^{\prime}$ and $G=\left[b_{j_{1}}, \ldots, b_{j_{s}}\right]$.

- Suppose $F=\varnothing$. Let $b_{i_{0}} \in \Delta_{p} \times\{1\}$ be the first vertex of $G$ and $a_{i_{0}}$ the vertex of $\Delta_{p} \times\{0\}$ whose projection on $\Delta_{p}$ is equal to the projection of $b_{i_{0}}$. We have $\Theta_{\Delta}\left(\mathbf{1}_{(G, \varepsilon)}\right)=0$. To determine the second term, first note,

$$
\begin{aligned}
\mathbf{1}_{(G, \varepsilon)} * a_{i_{0}} & =(-1)^{|(G, \varepsilon)|_{>p}}\left(\mathbf{1}_{\left(G_{p}, \varepsilon_{p}\right)} * a_{i_{0}}\right) \otimes \ldots \\
& =(-1)^{|(G, \varepsilon)|_{>p}+1}\left(\mathbf{1}_{\left(a_{i_{0}} * G_{p}, \varepsilon_{p}\right)}\right) \otimes \ldots
\end{aligned}
$$

It follows

$$
\begin{aligned}
\Theta_{\Delta} \delta \mathbf{1}_{(G, \varepsilon)} & =(-1)^{|(G, \varepsilon)|} \Theta_{\Delta}\left(\mathbf{1}_{(G, \varepsilon)} * a_{i_{0}}\right) \\
& =(-1)^{\left|\left(G_{p}, \varepsilon_{p}\right)\right|+1} \Theta_{\Delta}\left(\mathbf{1}_{\left(a_{i_{0}} * G, \varepsilon\right)}\right)=-\mathbf{1}_{(G, \varepsilon)} .
\end{aligned}
$$

- The proof for the case $G=\varnothing$ is similar.

Lemma 11.2. Let $\mathbf{1}_{(F * G, \varepsilon)} \in \tilde{N}^{*}(\Delta \otimes[0,1])$, with $F \neq \varnothing$ and $G \neq \varnothing$.

1) Let $\ell \in\{1, \ldots, n\}$. For any vertex $e \in \Delta_{\ell}$, we denote $e_{0} \in \Delta_{\ell} \times\{0\}$ and $e_{1} \in$ $\Delta_{\ell} \times\{1\}$ the vertices of $\Delta \otimes[0,1]$ corresponding to $e$. Then we have

$$
\Theta_{\Delta}\left(\mathbf{1}_{(F * G, \varepsilon)}\right) * e=\Theta_{\Delta}\left(\mathbf{1}_{(F * G, \varepsilon)} * e_{0}\right)+\Theta_{\Delta}\left(\mathbf{1}_{(F * G, \varepsilon)} * e_{1}\right) .
$$

2) For any virtual vertex $\mathrm{v}_{\ell}, \ell<n$, we have

$$
\Theta_{\Delta}\left(\mathbf{1}_{(F * G, \varepsilon)} * \mathrm{v}_{\ell}\right)=\left(\Theta_{\Delta}\left(\mathbf{1}_{(F * G, \varepsilon)}\right)\right) * \mathrm{v}_{\ell} .
$$


Proof. Let $\mathbf{1}_{(F * G, \varepsilon)}=\mathbf{1}_{\left(F_{0}, \varepsilon_{0}\right)} \otimes \cdots \otimes \mathbf{1}_{\left(F_{p} * G_{p}, \varepsilon_{p}\right)} \otimes \cdots \otimes \mathbf{1}_{\left(G_{n-1}, \varepsilon_{n-1}\right)} \otimes \mathbf{1}_{G_{n}}$.

$1) \bullet$ Let $\ell<p$. Using the Definition 2.9. we get $\Theta_{\Delta}\left(\mathbf{1}_{(F * G, \varepsilon)} * e_{1}\right)=0$ and the following equalities

$$
\begin{aligned}
& \Theta_{\Delta}\left(\mathbf{1}_{(F * G, \varepsilon)} * e_{0}\right)=
\end{aligned}
$$

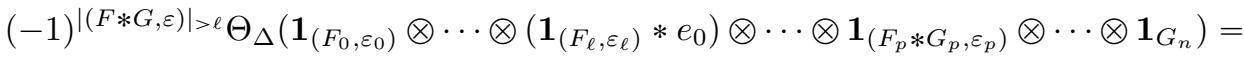

$$
\begin{aligned}
& (-1)^{|(F * G, \varepsilon)|_{>\ell}+|(F, \varepsilon)|_{<p}+1+\left|G_{p}\right|+\varepsilon_{p}} \cdots \otimes\left(\mathbf{1}_{\left(F_{\ell}, \varepsilon_{\ell}\right)} * e\right) \otimes \cdots \otimes \mathbf{1}_{\left(F_{p} \cup G_{p}, \varepsilon_{p}\right)} \otimes \cdots= \\
& (-1)^{|(F, \varepsilon)|_{<p}+\left|G_{p}\right|+\varepsilon_{p}}\left(\mathbf{1}_{\left(F_{0}, \varepsilon_{0}\right)} \otimes \cdots \otimes \mathbf{1}_{\left(F_{p} \cup G_{p}, \varepsilon_{p}\right)} \otimes \cdots \otimes \mathbf{1}_{G_{n}}\right) * e=\left(\Theta_{\Delta}\left(\mathbf{1}_{(F * G, \varepsilon)}\right)\right) * e .
\end{aligned}
$$

- The argument is the same if $\ell>p$.

- If $\ell=p$, these formulas become:

$$
\begin{aligned}
& \Theta_{\Delta}\left(\mathbf{1}_{(F * G, \varepsilon)} * e_{0}\right)=(-1)^{|(F * G, \varepsilon)|_{>p} \Theta_{\Delta}}\left(\mathbf{1}_{\left(F_{0}, \varepsilon_{0}\right)} \otimes \cdots \otimes \mathbf{1}_{\left(F_{p} * G_{p}, \varepsilon_{p}\right)} * e_{0} \otimes \cdots \otimes \mathbf{1}_{G_{n}}\right) . \\
& =(-1)^{|(F * G, \varepsilon)| \geqslant p+\varepsilon_{p}} \Theta_{\Delta}\left(\mathbf{1}_{\left(F_{0}, \varepsilon_{0}\right)} \otimes \cdots \otimes \mathbf{1}_{\left(F_{p} * G_{p} * e_{0}, \varepsilon_{p}\right)} \otimes \cdots \otimes \mathbf{1}_{G_{n}}\right) .
\end{aligned}
$$

Write $F_{p}=\left[a_{i_{0}}, \ldots, a_{i_{r}}\right], G_{p}=\left[b_{j_{0}}, \ldots, b_{j_{s}}\right]$. If the simplices are not compatible, both members of the previous equality are zero. The only cases giving compatible simplices are one of the following cases:

$$
(*) a_{i_{t}}<e<a_{i_{t+1}} \text { with } t \in\{0, \ldots, r-1\}, \quad(*) e<a_{i_{0}} \text { and } e \in \Delta_{p} .
$$

We treat the first case, the second being identical. Notice:

$$
\begin{aligned}
F_{p} * G_{p} * e & =\left[a_{i_{0}}, \ldots, a_{i_{r}}\right] *\left[b_{j_{0}}, \ldots, b_{j_{s}}\right] * e \\
& =(-1)^{s+1+r-t}\left[a_{i_{0}}, \ldots, a_{i_{t}}, e, a_{i_{t+1}}, \ldots, a_{i_{r}}\right] *\left[b_{j_{0}}, \ldots, b_{j_{s}}\right]
\end{aligned}
$$

and, setting $F_{p(e)}=\left[a_{i_{0}}, \ldots, a_{i_{t}}, e, a_{i_{t+1}}, \ldots, a_{i_{r}}\right]$,

$F_{p}(e) \cup G_{p}=\left[a_{i_{0}}, \ldots, a_{i_{t}}, e, a_{i_{t+1}}, \ldots, a_{i_{r}}=b_{j_{0}}, \ldots, b_{j_{s}}\right]=(-1)^{s+r-t}\left(F_{p} \cup G_{p}\right) * e$.

It follows

$$
\begin{array}{r}
\Theta_{\Delta}\left(\mathbf{1}_{(F * G, \varepsilon)} * e_{0}\right)= \\
(-1)^{|(F * G, \varepsilon)| \geqslant p+|(F, \varepsilon)|_{<p}+\left|G_{p}\right|+1} \mathbf{1}_{\left(F_{0}, \varepsilon_{0}\right)} \otimes \cdots \otimes \mathbf{1}_{\left(\left(F_{p} \cup G_{p}\right) * e, \varepsilon_{p}\right)} \otimes \cdots \otimes \mathbf{1}_{G_{n}}= \\
(-1)^{|(F, \varepsilon)|_{<p}+\left|G_{p}\right|+\varepsilon_{p}}\left(\mathbf{1}_{\left(F_{0}, \varepsilon_{0}\right)} \otimes \cdots \otimes\left(\mathbf{1}_{\left(F_{p} \cup G_{p}, \varepsilon_{p}\right)}\right) \otimes \cdots \otimes \mathbf{1}_{G_{n}}\right) * e= \\
\left(\Theta_{\Delta}\left(\mathbf{1}_{\left(F_{0}, \varepsilon_{0}\right)} \otimes \cdots \otimes\left(\mathbf{1}_{\left(F_{p} * G_{p}, \varepsilon_{p}\right)}\right) \otimes \cdots \otimes \mathbf{1}_{G_{n}}\right)\right) * e .
\end{array}
$$

In the case (11.3), we have $\Theta_{\Delta}\left(\mathbf{1}_{(F * G, \varepsilon)} * e_{1}\right)=0$, which gives the desired equality.

Similar argument gives the result in the symmetric case of the previous one: " $b_{j_{t}}<$ $e<b_{j_{t+1}}$ with $t \in\{1, \ldots, s-1\}$ or $b_{j_{s}}<e$ with $e \in \Delta_{p} . "$

2) We now consider a virtual vertex $v_{\ell}$. If $\varepsilon_{\ell}=1$, equality (11.2) is verified since its two members are zero. We therefore assume $\varepsilon_{\ell}=0$. If $\ell<p$, we have:

$$
\begin{array}{r}
\Theta_{\Delta}\left(\mathbf{1}_{(F * G, \varepsilon)} * \mathrm{v}_{\ell}\right)= \\
(-1)^{|(F * G, \varepsilon)|_{>\ell}+|(F, \varepsilon)|_{<p}+1+\left|\left(G_{p}, \varepsilon_{p}\right)\right|} \cdots \otimes\left(\mathbf{1}_{\left(F_{\ell}, 0\right)} * \mathrm{v}_{\ell}\right) \otimes \cdots \otimes \mathbf{1}_{\left(F_{p} \cup G_{p}, \varepsilon_{p}\right)} \otimes \cdots= \\
(-1)^{|(F, \varepsilon)|_{<p}+\left|\left(G_{p}, \varepsilon_{p}\right)\right|}\left(\mathbf{1}_{\left(F_{0}, \varepsilon_{0}\right)} \otimes \cdots \otimes \mathbf{1}_{\left(F_{p} \cup G_{p}, \varepsilon_{p}\right)} \otimes \cdots \otimes \mathbf{1}_{G_{n}}\right) * \mathrm{v}_{\ell}= \\
\left(\Theta_{\Delta}\left(\mathbf{1}_{(F * G, \varepsilon)}\right)\right) * \mathrm{v}_{\ell} .
\end{array}
$$

The argument is similar for $\ell \geqslant p$. 
Proposition 11.3. Let $X$ be a filtered space and let $X \times \mathbb{R}$ endowed with the product filtration and a perversity $\bar{p}$. Denoting also by $\bar{p}$ the induced perversity on $X$, there exists a linear map $\Theta: \widetilde{N}_{\bar{p}}^{*}(X \times \mathbb{R} ; R) \rightarrow \widetilde{N}_{\bar{p}}^{*-1}(X \times \mathbb{R} ; R)$, such that

$$
\Theta \circ \delta+\delta \circ \Theta=\left(I_{0} \circ \mathrm{pr}\right)^{*}-\mathrm{id} .
$$

Proof. Let $\sigma: \Delta \rightarrow X \times \mathbb{R}$ be a regular simplex and $\omega \in \tilde{N}^{k}(X \times \mathbb{R} ; R)$. We define $\hat{\sigma}: \mathcal{L}_{\Delta}=\Delta \otimes[0,1] \rightarrow X \times \mathbb{R}$ by $\hat{\sigma}(x, t)=\left(\operatorname{pr}(\sigma(x)), t \operatorname{pr}_{2}(\sigma(x))\right)$ and we set

$$
\omega_{\Delta \otimes[0,1]}=\sum_{(F * G, \varepsilon) \triangleleft \Delta \widetilde{\otimes[0,1]}} \omega_{\hat{\sigma} \circ \iota_{F * G}}(F * G, \varepsilon) \mathbf{1}_{(F * G, \varepsilon)},
$$

where $\iota_{F * G}: F * G \hookrightarrow \Delta \otimes[0,1]$ is the canonical injection. By construction, $\omega_{\Delta \otimes[0,1]} \in \tilde{N}^{k}(\Delta \otimes[0,1])$, and we can define

$$
\Theta(\omega)_{\sigma}=\Theta_{\Delta}\left(\omega_{\Delta \otimes[0,1]}\right) \in \tilde{N}^{k-1}(\Delta) .
$$

We need to verify $\Theta(\omega) \in \tilde{N}^{*}(X \times \mathbb{R} ; R)$. For this purpose, we consider a regular face operator $\delta_{\ell}: \nabla \rightarrow \Delta$ and $\tau=\sigma \circ \delta_{\ell}$. The property $\omega_{\Delta \otimes[0,1]} \in \tilde{N}^{*}(\Delta \otimes[0,1])$ becomes $\delta_{\ell}^{*} \omega_{\Delta \otimes[0,1]}=\omega_{\nabla \otimes[0,1]}$. The map $\delta_{\ell}^{*}: \tilde{N}^{*}(\Delta \otimes[0,1]) \rightarrow \tilde{N}^{*}(\nabla \otimes[0,1])$ verifies

$$
\delta_{\ell}^{*}\left(\mathbf{1}_{(F * G, \varepsilon)}\right)=\left\{\begin{array}{cl}
\mathbf{1}_{(F * G, \varepsilon)} & \text { if } F * G \triangleleft \nabla \otimes[0,1], \\
0 & \text { if not, }
\end{array}\right.
$$

which implies the commutativity of the following diagram,

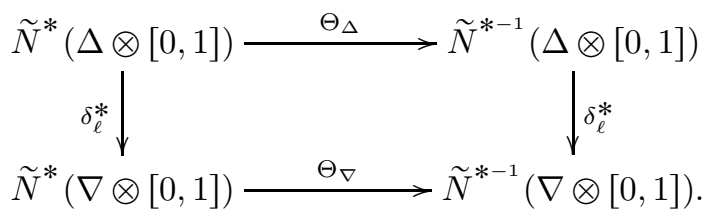

We deduce

$$
\delta_{\ell}^{*}\left(\Theta(\omega)_{\sigma}\right)=\delta_{\ell}^{*} \Theta_{\Delta}\left(\omega_{\Delta \otimes[0,1]}\right)=\Theta_{\nabla}\left(\delta_{\ell}^{*} \omega_{\Delta \otimes[0,1]}\right)=\Theta_{\nabla}\left(\omega_{\nabla \otimes[0,1]}\right)=\Theta(\omega)_{\tau} .
$$

The map $\Theta: \tilde{N}^{*}(X \times \mathbb{R} ; R) \rightarrow \widetilde{N}^{*-1}(X \times \mathbb{R} ; R)$ is well defined. From Proposition 11.1, we can deduce

$$
(\Theta \delta+\delta \Theta)(\omega)_{\sigma}=\sum_{(F, \varepsilon) \triangleleft \Delta \times\{0\}} \omega_{\hat{\sigma} \circ \iota_{F}}(F, \varepsilon) \mathbf{1}_{(F, \varepsilon)}-\sum_{(G, \varepsilon) \triangleleft \Delta \times\{1\}} \omega_{\hat{\sigma} \circ \iota_{G}}(G, \varepsilon) \mathbf{1}_{(G, \varepsilon)} .
$$

Recall the canonical inclusions $\iota_{0}, \iota_{1}: \Delta \rightarrow \Delta \otimes[0,1]$. If $F$ is a face of $\Delta \times\{0\}$ identified to $\nabla \triangleleft \Delta$, one has $\iota_{F}=\iota_{0} \circ \iota_{\nabla}$, from where

$$
\hat{\sigma} \circ \iota_{F}=\hat{\sigma} \circ \iota_{0} \circ \iota_{\nabla}=I_{0} \circ \operatorname{pr} \circ \sigma \circ \iota_{\nabla}
$$

and

$$
\sum_{(F, \varepsilon) \triangleleft \Delta \times\{0\}} \omega_{\hat{\sigma} \circ \iota_{F}}(F, \varepsilon) \mathbf{1}_{(F, \varepsilon)}=\left(I_{0} \circ \mathrm{pr}\right)^{*}\left(\sum_{(\nabla, \varepsilon) \triangleleft \tilde{\Delta}} \omega_{\sigma \circ \iota_{\nabla}}(\nabla, \varepsilon) \mathbf{1}_{(\nabla, \varepsilon)}\right)=\left(I_{0} \circ \mathrm{pr}\right)^{*}(\omega)_{\sigma} .
$$

Using $\iota_{G}=\iota_{1} \circ \iota_{\nabla}$ and $\hat{\sigma} \circ \iota_{G}=\hat{\sigma} \circ \iota_{1} \circ \iota_{\nabla}=\sigma \circ \iota_{\nabla}$, we show, as above, that the right side-hand of the second term of (11.5) is equal to $\omega_{\sigma}$. In summary, we have shown,

$$
(\Theta \delta+\delta \Theta)(\omega)_{\sigma}=\left(I_{0} \circ \mathrm{pr}\right)^{*}(\omega)_{\sigma}-\omega_{\sigma}
$$


from which comes the equality (11.4).

As for the compatibility with the perverse degrees, we prove $\|\Theta(\omega)\| \leqslant\|\omega\|$, for each $\omega \in \widetilde{N}^{*}(X \times \mathbb{R} ; R)$, by arguing over the chosen basis. This inequality follows directly from the definition of $\Theta$,

$$
\left\|\Theta_{\Delta}\left(\mathbf{1}_{(F * G, \varepsilon)}\right)\right\|_{\ell}=\left\|\mathbf{1}_{(F \cup G, \varepsilon)}\right\|_{\ell} \leqslant\left\|\mathbf{1}_{(F * G, \varepsilon)}\right\|_{\ell},
$$

for each $\ell \in\{1, \ldots, n\}$. With (11.4), we have constructed the homotopy $\Theta: \tilde{N}_{\bar{p}}^{*}(X \times \mathbb{R} ; R) \rightarrow \tilde{N}_{\bar{p}}^{*-1}(X \times \mathbb{R} ; R)$.

Proof of Theorem D. This is a direct consequence of the equalities pro $I_{0}=$ $\operatorname{pr} \circ I_{1}=\mathrm{id}$ and $\mathscr{H}_{\bar{p}}^{*}\left(I_{0} \circ \mathrm{pr}\right)=\mathrm{id}$, proved in (11.4).

The following result is used in Section 14

Proposition 11.4. Let $(X, \bar{p})$ be a paracompact perverse space. Let $S^{\ell}$ be the unit sphere of $\mathbb{R}^{\ell+1}$. The canonical projection $p_{X}: S^{\ell} \times X \rightarrow X,(z, x) \mapsto x$, induces an isomorphism $\mathscr{H}_{\bar{p}}^{k}\left(S^{\ell} \times X ; R\right) \cong \mathscr{H}_{\bar{p}}^{k}(X ; R) \oplus \mathscr{H}_{\bar{p}}^{k-\ell}(X ; R)$.

Proof. It suffices to use an induction on $\ell$ with the decomposition $S^{\ell}=$ $S^{\ell} \backslash\{$ Northpole $\} \cup S^{\ell} \backslash\{$ Southpole $\}$ (see Theorem $\mathrm{C}$ and Theorem D).

\section{Blown-up intersection cohomology of a cone. Theorem E,}

In this section, $X$ is an $n$-dimensional compact filtered space and we represent the open cone as the quotient $\stackrel{\circ}{\mathrm{c}} X=X \times[0, \infty[/ X \times\{0\}$, whose apex is denoted by w. The formal dimension of $\stackrel{\circ}{ } X$ is $n+1$ relatively to the conical filtration, $(\stackrel{\circ}{\mathrm{c}} X)_{i}={ }_{\mathrm{c}} X_{i-1}$ if $i \geqslant 1$ and $(\stackrel{\circ}{\mathrm{c}} X)_{0}=\{\mathrm{w}\}$. The purpose of this section is to prove the following proposition, cf. also [4, Corollary 1.47] and [24, Proposition 3.1.1].

Theorem E. Let $X$ be a compact filtered space. Consider the open cone, $\stackrel{\circ}{ } X=X \times[0, \infty[/ X \times\{0\}$, equipped with the conical filtration and a perversity $\bar{p}$. We also denote by $\bar{p}$ the perversity induced on $X$. The following properties are verified for any commutative ring, $R$.

(a) The inclusion $\iota: X \rightarrow \stackrel{\mathrm{c} X}{ }, x \mapsto[x, 1]$, induces an isomorphism, $\mathscr{H}_{\bar{p}}^{k}(\stackrel{\circ}{\mathrm{C}} X ; R) \stackrel{\cong}{\longrightarrow}$ $\mathscr{H}_{\bar{p}}^{k}(X ; R)$, for each $k \leqslant \bar{p}(\mathrm{w})$.

(b) For each $k>\bar{p}(\mathrm{w})$, we have $\mathscr{H}_{\bar{p}}^{k}(\stackrel{\circ}{\mathrm{c}} X ; R)=0$.

12.1. Simplices on a filtered space and its cone. First we link the complexes of $X$ and $\stackrel{\circ}{ } X$. The formal dimension of the cone being different from that of the original space, we introduce some operations which increase or decrease the length of filtrations.

- If $\Delta=\Delta_{0} * \cdots * \Delta_{n+1}$ is a regular simplex, of formal dimension $n+1$, we define a regular simplex, of formal dimension $n$, by $\widehat{\Delta}=\Delta_{1} * \cdots * \Delta_{n+1}$. Its filtration is characterized by $\widehat{\Delta}_{i}=\Delta_{i+1}$, for each $i \in\{0, \ldots, n\}$.

- Let $\sigma: \Delta_{\sigma}=\Delta_{0} * \cdots * \Delta_{n+1} \rightarrow \stackrel{\circ}{ } X$ be a regular simplex of $\stackrel{\circ}{ } X$. Since $\left.\sigma\left(\widehat{\Delta}_{\sigma}\right) \subset X \times\right] 0, \infty[$, we define the restriction

$$
\left.\hat{\sigma}: \Delta_{\hat{\sigma}}=\widehat{\Delta}_{\sigma} \stackrel{\sigma}{\rightarrow} X \times\right] 0, \infty[.
$$


- For each regular simplex of $\stackrel{\circ}{\mathrm{C}} X, \sigma: \Delta_{\sigma}=\Delta_{0} * \cdots * \Delta_{n+1} \rightarrow \stackrel{\circ}{\mathrm{c}} X$, the image of a point $x \in \Delta_{\sigma}$ can be written as,

$$
\sigma(x)=\left[\sigma_{1}(x), \sigma_{2}(x)\right] \in \stackrel{\circ}{\circ} X=X \times[0, \infty[/ X \times\{0\} .
$$

Associated to the simplex $\sigma$, there is the following regular simplex of $\stackrel{\circ}{ } X$,

$$
\mathrm{c} \sigma: \Delta_{\mathrm{c} \sigma}=\left(\{\mathrm{p}\} * \Delta_{0}\right) * \cdots * \Delta_{n+1} \rightarrow \stackrel{\circ}{\mathrm{c} X}
$$

defined by $\mathrm{c} \sigma((1-t) \mathrm{p}+t x)=\left[\sigma_{1}(x), t \sigma_{2}(x)\right]$. Moreover, if one considers $\left.\hat{\sigma}: \widehat{\Delta}_{\sigma} \rightarrow X \times\right] 0, \infty[\hookrightarrow \subset \mathrm{c} X$ as a filtered simplex of the cone, then $\mathrm{c} \hat{\sigma}$ is a face of $\mathrm{c} \sigma$.

The truncation of a cochain complex is defined for all positive integers $s$ by

$$
\left(\tau_{\leqslant s} C\right)^{r}=\left\{\begin{array}{ccc}
C^{r} & \text { if } & r<s, \\
\mathcal{Z} C^{s} & \text { if } & r=s, \\
0 & \text { if } & r>s,
\end{array}\right.
$$

where $\mathcal{Z} C^{s}$ means the $R$-module of cocycles whose degree is $s$.

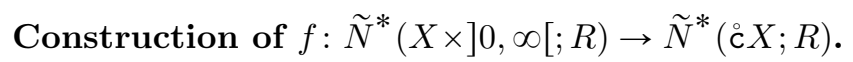

Let $\sigma: \Delta_{\sigma}=\Delta_{0} * \cdots * \Delta_{n+1} \rightarrow \stackrel{\complement}{ } X$ and $\omega \in \tilde{N}^{*}(X \times] 0, \infty[; R)$. We denote by $\lambda_{c \Delta_{0}}$ the cocycle $\mathbf{1}_{(\varnothing, 1)}+\sum_{e \in \mathcal{V}\left(\Delta_{0}\right)} \mathbf{1}_{([e], 0)} \in N^{0}\left(\mathrm{c} \Delta_{0}\right)$. We set

$$
f(\omega)_{\sigma}=\lambda_{c \Delta_{0}} \otimes \omega_{\hat{\sigma}} .
$$

Proposition 12.1. Let $(\stackrel{\circ}{\mathrm{c} X}, \bar{p})$ be a perverse space over the cone of the compact space $X$ and let $(X \times] 0, \infty[, \bar{p})$ be the induced perverse space. The correspondence defined above induces a cochain map,

$$
f: \tau_{\leqslant \bar{p}(\text { w })} \tilde{N}_{\bar{p}}^{*}(X \times] 0, \infty[; R) \rightarrow \tau_{\leqslant \bar{p}(\text { w })} \tilde{N}_{\bar{p}}^{*}(\stackrel{\circ}{\mathrm{c}} X ; R) .
$$

Proof. First, we check that the application $f$, defined locally at the level of simplices, extends globally to $\tilde{N}^{*}(X \times] 0, \infty[; R)$. For this, we must establish $\delta_{k}^{*} f(\omega)_{\sigma}=f(\omega)_{\sigma \circ \delta_{k}}$, for each $\omega \in \tilde{N}^{*}(X \times] 0, \infty[; R)$, each regular simplex, $\sigma: \Delta_{\sigma}=$ $\Delta_{0} * \cdots * \Delta_{n+1} \rightarrow \stackrel{\circ}{\mathrm{c} X}$, and any regular face operator, $\delta_{k}: \nabla \rightarrow \Delta_{\sigma}$, with $k \in$ $\left\{0, \ldots, \operatorname{dim} \Delta_{\sigma}\right\}$. Let $j_{0}$ denote the dimension of $\Delta_{0}$. To determine the effect of $\delta_{k}$ on the operation $\sigma \mapsto \hat{\sigma}$, we must distinguish $k>j_{0}$ of $k \leqslant j_{0}$. For the sake of convenience, we set $\delta_{s}=$ id if $s<0$. From the construction of $\hat{\sigma}$, we have

$$
\widehat{\sigma \circ \delta_{k}}=\left\{\begin{array}{ccc}
\hat{\sigma} \circ \delta_{k-j_{0}-1} & \text { if } & k>j_{0}, \\
\hat{\sigma} & \text { if } & k \leqslant j_{0}
\end{array}\right.
$$

which implies $\widehat{\sigma \circ \delta_{k}}=\hat{\sigma} \circ \delta_{k-j_{0}-1}$, with the previous convention. We conclude

$$
\delta_{k}^{*} f(\omega)_{\sigma}=\delta_{k}^{*}\left(\lambda_{c \Delta_{0}} \otimes \omega_{\hat{\sigma}}\right)=\left\{\begin{array}{ccc}
\lambda_{c \Delta_{0}} \otimes \delta_{k-j_{0}-1}^{*} \omega_{\hat{\sigma}} & \text { if } & k>j_{0}, \\
\lambda_{c} \nabla_{0} \otimes \omega_{\hat{\sigma}} & \text { if } & k \leqslant j_{0} .
\end{array}\right.
$$

It follows $\delta_{k}^{*} f(\omega)_{\sigma}=\lambda_{c \nabla_{0}} \otimes \omega_{\widehat{\sigma \circ \delta_{k}}}=f(\omega)_{\sigma \circ \delta_{k}}$.

Since the 0-cochain $\lambda_{c \Delta_{0}}$ is a cocycle, the compatibility with the differentials is immediate from the equalities

$$
\delta\left(f(\omega)_{\sigma}\right)=\delta\left(\lambda_{c \Delta_{0}} \otimes \omega_{\hat{\sigma}}\right)=\lambda_{c \Delta_{0}} \otimes \delta \omega_{\hat{\sigma}}=f(\delta \omega)_{\sigma} .
$$

The map $f$ being compatible with the differentials, it remains to show that the image by $f$ of a $\bar{p}$-allowable cochain, $\omega \in \widetilde{N}^{*}(X \times] 0, \infty[; R)$, is a $\bar{p}$-allowable cochain in $\tilde{N}^{*}(\stackrel{c}{\mathrm{c} X} ; R)$. We choose $\omega$ of degree less than or equal to $\bar{p}(\mathrm{w})$ and refer to Definition 3.4 for the property of $\bar{p}$-allowability. For the stratum reduced to 
w, the allowability comes directly from $\left\|f(\omega)_{\sigma}\right\|_{n+1} \leqslant\left|\omega_{\hat{\sigma}}\right| \leqslant \bar{p}(\mathrm{w})$. Now consider a singular stratum $S$ of $X$ and a regular simplex $\sigma: \Delta_{\sigma}=\Delta_{0} * \cdots * \Delta_{n+1} \rightarrow \stackrel{\circ}{ } X$, such that $\sigma\left(\Delta_{\sigma}\right) \cap(S \times] 0, \infty[) \neq \varnothing$. Let $\ell=\operatorname{codim}_{X \times] 0, \infty[}(S \times] 0, \infty[)$ and notice the equivalence of the conditions $\sigma\left(\Delta_{\sigma}\right) \cap(S \times] 0, \infty[) \neq \varnothing$ and $\hat{\sigma}\left(\Delta_{\hat{\sigma}}\right) \cap(S \times] 0, \infty[) \neq \varnothing$. For such stratum, we have $\ell \in\{1, \ldots, n\}$ and $\left\|f(\omega)_{\sigma}\right\|_{\ell}=\left\|\lambda_{c \Delta_{0}} \otimes \omega_{\hat{\sigma}}\right\|_{\ell}=\left\|\omega_{\hat{\sigma}}\right\|_{\ell}$. The result is a consequence of the inequality $\left\|\omega_{\hat{\sigma}}\right\|_{\ell} \leqslant\|\omega\|_{S \times] 0, \infty} \leqslant \bar{p}(S \times] 0, \infty[$, arising from the $\bar{p}$-allowability of $\omega$.

Construction of $g: \tilde{N}^{*}(\stackrel{\circ}{\mathrm{C}} ; R) \rightarrow \tilde{N}^{*}(X \times] 0, \infty[; R)$.

Let $\omega \in \tilde{N}^{*}(\stackrel{\circ}{\mathrm{c}} X ; R)$ and $\left.\tau: \Delta_{\tau} \rightarrow X \times\right] 0, \infty[$ a regular simplex. We denote by $\mathrm{c} \tau: \Delta_{c \tau}=\{\mathrm{p}\} * \Delta_{\tau} \rightarrow \stackrel{\mathrm{c} X}{ } X$ the cone over $\tau$ defined above. Notice $\widetilde{\Delta_{\mathrm{c} \tau}}=\mathrm{c}\{\mathrm{p}\} \times \widetilde{\Delta_{\tau}}$.

Let $\mathrm{v}_{0}$ be the apex of the cone over the component of filtration degree 0 of a filtered simplex. The cone $\mathrm{c}\{\mathrm{p}\}$ having two vertices $\mathrm{p}$ and $\mathrm{v}_{0}$, the cochain $\omega_{\mathrm{c} \tau}$ decomposes into

$$
\omega_{\mathrm{c} \tau}=\mathbf{1}_{\mathrm{p}} \otimes \gamma_{\mathrm{p}}+\mathbf{1}_{\mathrm{v}_{0}} \otimes \gamma_{\mathrm{v}_{0}}+\mathbf{1}_{\mathrm{p} * \mathrm{v}_{0}} \otimes \gamma_{\mathrm{v}_{0}}^{\prime},
$$

with $\gamma_{\mathrm{p}}, \gamma_{\mathrm{v}_{0}}, \gamma_{\mathrm{v}_{0}}^{\prime} \in \tilde{N}^{*}\left(\Delta_{\tau}\right)$. We set

$$
g(\omega)_{\tau}=\gamma_{\mathrm{v}_{0}} .
$$

Proposition 12.2. Let $(\stackrel{\circ}{\mathrm{c} X}, \bar{p})$ be a perverse space with $X$ compact and let $(X \times] 0, \infty[, \bar{p})$ be the induced perverse space. The correspondence defined above induces a cochain map,

$$
g: \tau_{\leqslant \bar{p}(\mathrm{w})} \tilde{N}_{\bar{p}}^{*}(\stackrel{\circ}{ } \mathrm{X} ; R) \rightarrow \tau_{\leqslant \bar{p}(\mathrm{w})} \tilde{N}_{\bar{p}}^{*}(X \times] 0, \infty[; R) .
$$

The proof follows the pattern of that of Proposition 12.1 we leave it to the reader.

Specify the compositions $f \circ g$ and $g \circ f$.

(a) Let $\omega \in \tilde{N}^{*}(X ; R)$. Consider a regular simplex, $\left.\tau: \Delta_{\tau} \rightarrow X \times\right] 0, \infty[$ and its associated map $\mathrm{c} \tau:\{\mathrm{p}\} * \Delta_{\tau} \rightarrow \stackrel{\mathrm{c} X}{ }$. Following (12.2), one has

$$
f(\omega)_{\mathrm{c} \tau}=\lambda_{\mathrm{c}\{\mathrm{p}\}} \otimes \omega_{\tau}=\mathbf{1}_{\mathrm{p}} \otimes \omega_{\tau}+\mathbf{1}_{\mathrm{v}_{0}} \otimes \omega_{\tau} .
$$

It follows, according to (12.3), $g(f(\omega))_{\tau}=\omega_{\tau}$ and $g \circ f=$ id.

(b) Let $\omega \in \tilde{N}^{*}(\stackrel{\circ}{\mathrm{c}} X ; R)$. Consider a regular simplex $\sigma: \Delta_{\sigma}=\Delta_{0} * \cdots * \Delta_{n+1} \rightarrow \stackrel{\circ}{\mathrm{c}} X$, and its associated map $\mathrm{c} \sigma:\left(\{\mathrm{p}\} * \Delta_{0}\right) * \cdots * \Delta_{n+1} \rightarrow \stackrel{\circ}{\mathrm{C} X}$. The cochain $\omega_{\mathrm{c} \sigma}$ decomposes into

$$
\omega_{\mathrm{c} \sigma}=\sum_{F \triangleleft c \Delta_{0}} \mathbf{1}_{F} \otimes \gamma_{F}+\sum_{F \triangleleft c \Delta_{0}} \mathbf{1}_{\mathrm{p} * F} \otimes \gamma_{F}^{\prime}+\mathbf{1}_{\mathrm{p}} \otimes \gamma_{\varnothing} .
$$

Since the cochain $\omega$ is globally defined and the simplex c $\hat{\sigma}$ is a face of $c \sigma$, we deduce $\omega_{\mathrm{c} \hat{\sigma}}=\mathbf{1}_{\mathrm{v}_{0}} \otimes \gamma_{\mathrm{v}_{0}}+\mathbf{1}_{\mathrm{p} * \mathrm{v}_{0}} \otimes \gamma_{\mathrm{v}_{0}}^{\prime}+\mathbf{1}_{\mathrm{p}} \otimes \gamma_{\varnothing}$. It follows:

$$
f(g(\omega))_{\sigma}=\lambda_{\mathrm{c} \Delta_{0}} \otimes g(\omega)_{\hat{\sigma}}=\lambda_{\mathrm{c} \Delta_{0}} \otimes \gamma_{\mathrm{v}_{0}} .
$$

Construction of a homotopy $H: \tilde{N}^{*}(\stackrel{\circ}{\mathrm{C}} X ; R) \rightarrow \tilde{N}^{*-1}(\stackrel{\circ}{\mathrm{c} X} ; R)$. 
If $\sigma: \Delta_{\sigma}=\Delta_{0} * \cdots * \Delta_{n+1} \rightarrow \stackrel{\circ}{\mathrm{C}} X$ is a regular simplex, we define a map $H: \tilde{N}^{*}\left(\Delta_{\mathrm{c} \sigma}\right) \rightarrow \tilde{N}^{*-1}\left(\Delta_{\sigma}\right)$, i.e.,

$H: N^{*}\left(\mathrm{c}\left(\mathrm{p} * \Delta_{0}\right)\right) \otimes N^{*}\left(\mathrm{c} \Delta_{1}\right) \otimes \cdots \otimes N^{*}\left(\Delta_{n+1}\right) \rightarrow N^{*-1}\left(\mathrm{c} \Delta_{0}\right) \otimes \cdots \otimes N^{*}\left(\Delta_{n+1}\right)$.

We decompose $\omega_{\mathrm{c} \sigma} \in \tilde{N}^{*}\left(\Delta_{\mathrm{c} \sigma}\right)$ as in the formula (12.4) and set:

$$
(H(\omega))_{\sigma}=\sum_{\mathrm{v}_{0} \neq F \triangleleft \mathrm{c} \Delta_{0}}(-1)^{|F|+1} \mathbf{1}_{F} \otimes \gamma_{F}^{\prime}+\lambda_{\Delta_{0}} \otimes \gamma_{\mathrm{v}_{0}}^{\prime},
$$

where $\mathrm{v}_{0}$ is the apex of the cone over the component filtration of degree 0 and $\lambda_{\Delta_{0}}$ the sum of 0-cochains on $\Delta_{0}$.

Proposition 12.3. Let $(\stackrel{\circ}{\mathrm{c}} X, \bar{p})$ be a perverse space with $X$ compact.

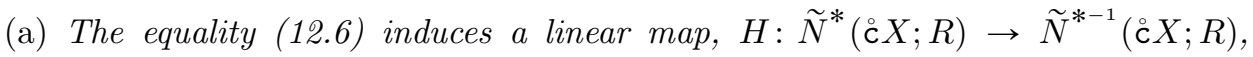
verifying

$$
\delta \circ H+H \circ \delta=\mathrm{id}-f \circ g .
$$

(b) Using the notation introduced in (12.1), the application $H$ induces a map,

$$
H: \tau_{\leqslant \bar{p}(\text { w) }} \tilde{N}_{\bar{p}}^{*}(\stackrel{\circ}{\mathrm{c}} X ; R) \rightarrow \tau_{\leqslant \bar{p}(\text { w) }} \tilde{N}_{\bar{p}}^{*-1}(\stackrel{\circ}{\mathrm{c}} X ; R) .
$$

Proof. (a) We must establish the equality $\delta_{k}^{*} H(\omega)_{\sigma}=H(\omega)_{\sigma \circ \delta_{k}}$, for each cochain $\omega \in \tilde{N}^{*}(\stackrel{\circ}{\mathrm{c} X})$, each regular simplex $\sigma: \Delta_{\sigma}=\Delta_{0} * \cdots * \Delta_{n+1} \rightarrow \stackrel{\circ}{ } X$ and each regular face operator $\delta_{k}: D=D_{0} * \cdots * D_{n+1} \rightarrow \Delta_{\sigma}$, with $k \in\left\{0, \ldots, \operatorname{dim} \Delta_{\sigma}\right\}$. Denoting by $\delta_{*}^{\mathrm{p}}$ the regular face operators of $\{\mathrm{p}\} * \Delta_{\sigma}$, we can write $\mathrm{c}\left(\sigma \circ \delta_{k}\right)=$ $\mathrm{c} \sigma \circ \delta_{k+1}^{\mathrm{p}}$. If $k>\operatorname{dim} \Delta_{0}$, we set $k^{\circ}=k-\operatorname{dim} \Delta_{0}-1$ and $\delta_{k^{\circ}}^{\circ}: D_{1} * \cdots * D_{n+1} \rightarrow$ $\Delta_{1} * \cdots * \Delta_{n+1}$ the induced face. Following (12.6), we have:

$$
\delta_{k}^{*} H(\omega)_{\sigma}=\left\{\begin{array}{cl}
\sum_{\mathrm{v}_{0} \neq F \triangleleft c D_{0}}(-1)^{|F|+1} \mathbf{1}_{F} \otimes \gamma_{F}^{\prime}+\lambda_{D_{0}} \otimes \gamma_{\mathrm{v}_{0}}^{\prime} & \text { if } k \leqslant \operatorname{dim} \Delta_{0}, \\
\sum_{\mathrm{v}_{0} \neq F \triangleleft c \Delta_{0}}(-1)^{|F|+1} \mathbf{1}_{F} \otimes \delta_{k^{\circ}}^{\circ, *} \gamma_{F}^{\prime}+\lambda_{\Delta_{0}} \otimes \delta_{k^{\circ}}^{\circ, *} \gamma_{\mathrm{v}_{0}}^{\prime} & \text { if } k>\operatorname{dim} \Delta_{0} .
\end{array}\right.
$$

Using the equality $\omega_{\mathrm{c}\left(\sigma \circ \delta_{k}\right)}=\omega_{\mathrm{c} \sigma \circ \delta_{k+1}^{\mathrm{p}}}=\delta_{k+1}^{\mathrm{p}, *} \omega_{\mathrm{c} \sigma}$ and (12.4), we get:

$\omega_{\mathrm{c}\left(\sigma \circ \delta_{k}\right)}=\left\{\begin{array}{cl}\left(\sum_{F \triangleleft c D_{0}} \mathbf{1}_{F} \otimes \gamma_{F}+\mathbf{1}_{\mathrm{p} * F} \otimes \gamma_{F}^{\prime}\right)+\mathbf{1}_{\mathrm{p}} \otimes \gamma_{\varnothing} & \text { if } k \leqslant \operatorname{dim} \Delta_{0}, \\ \left(\sum_{F \triangleleft c D_{0}} \mathbf{1}_{F} \otimes \delta_{k^{\circ}}^{\circ, *} \gamma_{F}+\mathbf{1}_{\mathrm{p} * F} \otimes \delta_{k^{\circ}}^{\circ, *} \gamma_{F}^{\prime}\right)+\mathbf{1}_{\mathrm{p}} \otimes \delta_{k^{\circ}}^{\circ} \gamma_{\varnothing} & \text { if } k>\operatorname{dim} \Delta_{0},\end{array}\right.$

which gives

$$
\begin{aligned}
H(\omega)_{\sigma \circ \delta_{k}} & =\left\{\begin{array}{cl}
\left(\sum_{\mathrm{v}_{0} \neq F \triangleleft c D_{0}}(-1)^{|F|+1} \mathbf{1}_{F} \otimes \gamma_{F}^{\prime}\right)+\lambda_{D_{0}} \otimes \gamma_{\mathrm{v}_{0}}^{\prime}, & \text { if } k \leqslant \operatorname{dim} \Delta_{0}, \\
\left(\sum_{\mathrm{v}_{0} \neq F \triangleleft c D_{0}}(-1)^{|F|+1} \mathbf{1}_{F} \otimes \delta_{k^{\circ}}^{\circ, *} \gamma_{F}^{\prime}\right)+\lambda_{D_{0}} \otimes \delta_{k^{\circ}}^{\circ *} \gamma_{\mathrm{v}_{0}}^{\prime} & \text { if } k>\operatorname{dim} \Delta_{0},
\end{array}\right. \\
& =\delta_{k}^{*} H(\omega)_{\sigma} .
\end{aligned}
$$

Let us study the behavior of $H$ towards the differentials. Let $\sigma: \Delta_{\sigma}=\Delta_{0} *$ $\ldots * \Delta_{n+1} \rightarrow \stackrel{\circ}{ } \times$ be a regular simplex and $\omega \in \tilde{N}^{*}(\stackrel{\circ}{\subset} X ; R)$. Let us start from the equality (12.4) and calculate the differential in eliminating terms having a zero 
image by $H$.

$$
\begin{aligned}
(12.7)(H(\delta \omega))_{\sigma}= & H\left(\sum_{e \in \mathcal{V}\left(c \Delta_{0}\right)} \mathbf{1}_{p * e} \otimes \gamma_{\varnothing}+\sum_{F \triangleleft c \Delta_{0}} \mathbf{1}_{F * \mathrm{p}} \otimes \gamma_{F}+\right. \\
& \left.\sum_{\substack{F \triangleleft c \Delta_{0} \\
e \in \mathcal{V}\left(c \Delta_{0}\right)}} \mathbf{1}_{\mathrm{p} * F * e} \otimes \gamma_{F}^{\prime}+\sum_{F \triangleleft c \Delta_{0}}(-1)^{|F|+1} \mathbf{1}_{\mathrm{p} * F} \otimes \delta \gamma_{F}^{\prime}\right) .
\end{aligned}
$$

Notice

$$
\begin{array}{r}
\sum_{e \in \mathcal{V}\left(\mathrm{c} \Delta_{0}\right)} \mathbf{1}_{p * e} \otimes \gamma_{\varnothing}+\sum_{F \triangleleft \mathrm{c} \Delta_{0}} \mathbf{1}_{F * \mathrm{p}} \otimes \gamma_{F}= \\
\sum_{e \in \mathcal{V}\left(\Delta_{0}\right)} \mathbf{1}_{p * e} \otimes \gamma_{\varnothing}+\sum_{\mathrm{v}_{0} \neq F \triangleleft c \Delta_{0}} \mathbf{1}_{F * \mathrm{p}} \otimes \gamma_{F}+\mathbf{1}_{\mathrm{p} * \mathrm{v}_{0}} \otimes\left(\gamma_{\varnothing}-\gamma_{\mathrm{v}_{0}}\right) .
\end{array}
$$

Replacing in (12.7) and developing the definition of $H$, we get:

$$
\begin{aligned}
(H(\delta \omega))_{\sigma}= & -\sum_{e \in \mathcal{V}\left(\Delta_{0}\right)} \mathbf{1}_{e} \otimes \gamma_{\varnothing}+\sum_{\mathrm{v}_{0} \neq F \triangleleft c \Delta_{0}} \mathbf{1}_{F} \otimes \gamma_{F}+\lambda_{\Delta_{0}} \otimes\left(\gamma \varnothing-\gamma_{\mathrm{v}_{0}}-\delta \gamma_{\mathrm{v}_{0}}^{\prime}\right) \\
& +\sum_{\substack{F \triangleleft c \Delta_{0} \\
e \in \mathcal{V}\left(\mathrm{c} \Delta_{0}\right)}}(-1)^{|F|} \mathbf{1}_{F * e} \otimes \gamma_{F}^{\prime}+\sum_{\mathrm{v}_{0} \neq F \triangleleft c \Delta_{0}} \mathbf{1}_{F} \otimes \delta \gamma_{F}^{\prime} .
\end{aligned}
$$

On the other hand, the quantity $\delta \circ H$ can be written

$$
\begin{aligned}
\delta(H(\omega))_{\sigma}= & \sum_{\substack{\mathrm{v}_{0} \neq F \triangleleft c \Delta_{0} \\
e \in \mathcal{V}\left(\mathrm{c} \Delta_{0}\right)}}(-1)^{|F|+1} \mathbf{1}_{F * e} \otimes \gamma_{F}^{\prime}-\sum_{\mathrm{v}_{0} \neq F \triangleleft c \Delta_{0}} \mathbf{1}_{F} \otimes \delta \gamma_{F}^{\prime} \\
& +\sum_{e \in \mathcal{V}\left(\Delta_{0}\right)} \mathbf{1}_{e * \mathrm{v}_{0}} \otimes \gamma_{\mathrm{v}_{0}}^{\prime}+\lambda_{\Delta_{0}} \otimes \delta \gamma_{\mathrm{v}_{0}}^{\prime} .
\end{aligned}
$$

Using (12.5), the sum of the two expressions can be reduced to:

$$
H(\delta \omega)_{\sigma}+\delta H(\omega)_{\sigma}=\sum_{F \triangleleft c \Delta_{0}} \mathbf{1}_{F} \otimes \gamma_{F}-\lambda_{c \Delta_{0}} \otimes \gamma_{\mathrm{v}_{0}}=\omega_{\sigma}-(f \circ g)(\omega)_{\sigma} .
$$

(b) As in the proof of Proposition 12.1, we are reduced to consider a singular

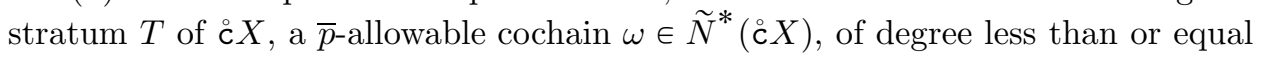
to $\bar{p}(\mathrm{w})$, and a regular simplex, $\sigma: \Delta_{\sigma} \rightarrow \stackrel{\mathrm{c} X}{ }$, with $\sigma\left(\Delta_{\sigma}\right) \cap T \neq \varnothing$. Let $\ell=$ $\operatorname{codim}_{c} X \in\{1, \ldots, n+1\}$. Notice that $c \sigma\left(\Delta_{c \sigma}\right) \cap T \neq \varnothing$. Thus, according to the definition of perverse degree (cf. Definition [2.5), we have

$$
\begin{aligned}
\bar{p}(T) & \geqslant\|\omega\|_{T} \geqslant\left\|\omega_{\mathrm{c} \sigma}\right\|_{\ell}=\max \left\{\left\|\mathbf{1}_{F} \otimes \gamma_{F}\right\|_{\ell},\left\|\mathbf{1}_{\mathrm{p} * F} \otimes \gamma_{F}^{\prime}\right\|_{\ell},\left\|\mathbf{1}_{\mathrm{p}} \otimes \gamma_{\varnothing}\right\|_{\ell} \mid F \triangleleft \mathrm{c} \Delta_{0}\right\} \\
& \geqslant \max \left\{\left\|\mathbf{1}_{\mathrm{p} * F} \otimes \gamma_{F}^{\prime}\right\|_{\ell} \mid F \triangleleft \mathrm{c} \Delta_{0}\right\},
\end{aligned}
$$

where the equality uses (2.5) and (12.4). We develop this expression by distinguishing two cases.

- Let $\ell \neq n+1$. By definition, for any face $F \triangleleft \check{c} \Delta_{0}$, we have the equality $\left\|\mathbf{1}_{\mathrm{p} * F} \otimes \gamma_{F}^{\prime}\right\|_{\ell}=\left\|\mathbf{1}_{F} \otimes \gamma_{F}^{\prime}\right\|_{\ell}$, if $F \neq \mathrm{v}_{0}$, and $\left\|\mathbf{1}_{\mathrm{p} * \mathrm{v}_{0}} \otimes \gamma_{\mathrm{v}_{0}}^{\prime}\right\|_{\ell}=\left\|\lambda_{\Delta_{0}} \otimes \gamma_{\mathrm{v}_{0}}^{\prime}\right\|_{\ell}$. It follows:

$\bar{p}(T) \geqslant \max \left\{\left\|\mathbf{1}_{F} \otimes \gamma_{F}^{\prime}\right\|_{\ell},\left\|\lambda_{\Delta_{0}} \otimes \gamma_{\mathrm{v}_{0}}^{\prime}\right\|_{\ell} \mid \mathrm{v}_{0} \neq F \triangleleft \mathrm{c} \Delta_{0}\right\}=\left\|H(\omega)_{\sigma}\right\|_{\ell}$, where the last equality uses (2.5) and (12.6). We deduce $\bar{p}(T) \geqslant\|H(\omega)\|_{T}$. 
- Let $\ell=n+1$. In this case we have

$$
\begin{aligned}
\left\|H(\omega)_{\sigma}\right\|_{n+1} & =\max \left\{\left\|\mathbf{1}_{\mathrm{p} * F} \otimes \gamma_{F}^{\prime}\right\|_{n+1},\left\|\lambda_{\Delta_{0}} \otimes \gamma_{\mathrm{v}_{0}}^{\prime}\right\|_{n+1} \mid \mathrm{v}_{0} \neq F \triangleleft \mathrm{c} \Delta_{0}\right\} \\
& =\max \left\{\left|\gamma_{F}^{\prime}\right|,\left|\gamma_{\mathrm{v}_{0}}^{\prime}\right| \mid F \triangleleft \Delta_{0}\right\} \leqslant|\omega|-1 \leqslant \bar{p}(\mathrm{w}),
\end{aligned}
$$

where the first equality uses (2.5) and (12.6). It follows $\bar{p}(\mathrm{w}) \geqslant\|H(\omega)\|_{\mathrm{w}}$.

It has been shown $\|H(\omega)\| \leqslant \bar{p}$. The property $\|\delta H(\omega)\| \leqslant \bar{p}$ is deduced using (12.8).

The determination of the cohomology of a cone follows from the properties of $f, g$ and $H$.

Proof of Theorem E. (a) From Propositions 12.1, 12.2 and 12.3, we deduce that the map $g: \tau_{\leqslant \bar{p}(\text { w) }} \tilde{N}_{\bar{p}}^{*}(\stackrel{\circ}{\mathrm{c}} X ; R) \rightarrow \tau_{\leqslant \bar{p}(\mathrm{w})} \tilde{N}_{\bar{p}}^{*}(X \times] 0, \infty[; R)$ is a quasi-isomorphism. We know from Theorem $\mathrm{D}$, that the inclusion $\left.I_{1}: X \rightarrow X \times\right] 0, \infty[$ induces a quasi-isomorphism. It remains to prove $I_{1}^{*} \circ g=\iota^{*}$. The involved stratifications on $X \times] 0, \infty[$ are different in these two quasi-isomorphisms but the cohomologies are the same (see subsection 3.1).

For this, consider $\omega \in \tau_{\bar{p}(\text { w })} \tilde{N}_{\bar{p}}^{*}(\stackrel{\circ}{\mathrm{c}} X ; R)$ and $\tau: \Delta_{\tau} \rightarrow X$ a regular simplex. By definition, we have $\left(\iota^{*} \omega\right)_{\tau}=\omega_{\iota \circ \tau}$. Notice $\iota \circ \tau=\mathrm{c}\left(I_{1} \circ \tau\right) \circ \delta_{0}$, where $\delta_{0}(x)=0 \cdot \mathrm{p}+1 \cdot x$. It then follows

$$
\left(\iota^{*} \omega\right)_{\tau}=\omega_{\mathrm{c}\left(I_{1} \circ \tau\right) \circ \delta_{0}}=\delta_{0}^{*} \omega_{\mathrm{c}\left(I_{1} \circ \tau\right)}=\underline{12.3}, \gamma_{\mathrm{v}_{0}}=g(\omega)_{I_{1} \circ \tau}=I_{1}^{*}(g(\omega))_{\tau} .
$$

To prove part (b), we consider a cocycle $\omega \in \tilde{N}_{\bar{p}}^{k}(\stackrel{\circ}{\mathrm{c} X} R)$ with $k>\bar{p}(\mathrm{w})$. Following Proposition 12.3, we have $\delta H(\omega)=\omega-f(g(\omega))$ and it suffices to establish the equality $f(g(\omega))=0$.

We prove it by contradiction, assuming that there is a regular simplex $\sigma: \Delta_{\sigma} \rightarrow$ $\stackrel{\complement}{ } X$, such that $f(g(\omega))_{\sigma} \neq 0$. Following (12.5), this implies $\gamma_{\mathrm{v}_{0}} \neq 0$. We get a contradiction,

$$
\begin{aligned}
k>\bar{p}(\mathrm{w}) & \geqslant\|f(g(\omega))\|_{\mathrm{w}} \geqslant\left\|f(g(\omega))_{\mathrm{c} \sigma}\right\|_{n+1}=\left\|\lambda_{\mathrm{c}\left(\mathrm{p} * \Delta_{0}\right)} \otimes \gamma_{\mathrm{v}_{0}}\right\|_{n+1} \\
& =\left\|\lambda_{\mathrm{p} * \Delta_{0}} \otimes \gamma_{\mathrm{v}_{0}}\right\|_{n+1}=\left|\gamma_{\mathrm{v}_{0}}\right|=k .
\end{aligned}
$$

The proof of the topological independence Theorem of Section 14 is based on the following excision theorem.

12.2. Relative cohomology. Let $X$ be a filtered space and $Y \subset X$ a subspace endowed with the induced stratification. Consider a perversity $\bar{p}$ on $X$. We also denote by $\bar{p}$ the induced perversity on $Y$. The complex of relative $\bar{p}$-intersection cochains is the direct sum $\widetilde{N}_{\bar{p}}^{*}(X, Y ; R)=\tilde{N}_{\bar{p}}^{*}(X ; R) \oplus \widetilde{N}_{\bar{p}}^{*-1}(Y ; R)$, endowed with the differential $D(\alpha, \beta)=(d \alpha, \alpha-d \beta)$. Its homology is the relative blown-up $\bar{p}$ intersection cohomology of the perverse pair $(X, Y, \bar{p})$, denoted by $\mathscr{H}_{\bar{p}}^{*}(X, Y ; R)$.

By definition, we have a long exact sequence associated to the perverse pair $(X, Y, \bar{p})$ :

$$
\ldots \rightarrow \mathscr{H}_{\bar{p}}^{i}(X ; R) \stackrel{1^{*}}{\rightarrow} \mathscr{H}_{\bar{p}}^{i}(Y ; R) \rightarrow \mathscr{H}_{\bar{p}}^{i+1}(X, Y ; R) \stackrel{\operatorname{pr}^{*}}{\rightarrow} \mathscr{H}_{\bar{p}}^{i+1}(X ; R) \rightarrow \ldots
$$

where $\operatorname{pr}: \widetilde{N}_{\bar{p}}^{*}(X, Y ; R) \rightarrow \widetilde{N}_{\bar{p}}^{*}(X ; R)$ is defined by $\operatorname{pr}(\alpha, \beta)=\alpha$ and $1^{*}$ comes from the restriction map 1: $\tilde{N}_{\bar{p}}^{*}(X ; R) \rightarrow \widetilde{N}_{\bar{p}}^{*}(Y ; R)$. 
Proposition 12.4. Let $(X, \bar{p})$ be a paracompact perverse space. If $F$ is a closed subset of $X$ and $U$ an open subset of $X$ with $F \subset U$, then the natural inclusion $(X \backslash F, U \backslash F) \hookrightarrow(X, U)$ induces the isomorphism

$$
\mathscr{H}_{\bar{p}}^{*}(X, U ; R) \cong \mathscr{H}_{\bar{p}}^{*}(X \backslash F, U \backslash F ; R) .
$$

Proof. For the sake of simplicity we write the complexes $\mathcal{A}^{*}=\tilde{N}_{\bar{p}}^{*, \mathcal{U}}(X ; R)$, $\mathcal{B}^{*}=\tilde{N}_{\bar{p}}^{*, \mathcal{U}_{1}}(X \backslash F ; R), \mathcal{C}^{*}=\tilde{N}_{\bar{p}}^{*, \mathcal{U}_{2}}(U ; R)$ and $\mathcal{E}^{*}=\tilde{N}_{\bar{p}}^{*}(U \backslash F ; R)$, the terms of the Mayer-Vietoris exact sequence of Theorem $\mathbb{C}$ applied to the cover $\mathcal{U}=\{X \backslash F, U\}$. Associated to this sequence we have the exact sequence

$$
0 \rightarrow\left(\mathcal{A}^{*} \oplus \mathcal{C}^{*-1}, D\right) \stackrel{f}{\rightarrow}\left(\mathcal{B}^{*} \oplus \mathcal{C}^{*} \oplus \mathcal{C}^{*-1} \oplus \mathcal{E}^{*-1}, D^{\prime}\right) \stackrel{g}{\rightarrow}\left(\mathcal{E}^{*} \oplus \mathcal{E}^{*-1}, D\right) \rightarrow 0
$$

where $D(a, c)=(d a, a-d c), f(a, c)=(a, a, c, 0), D^{\prime}\left(b, c_{0}, c_{1}, e\right)=\left(d b, d c_{0}, c_{0}-\right.$ $\left.d c_{1}, b-c_{0}-d e\right), g\left(b, c_{0}, c_{1}, e\right)=\left(b-c_{0}, e\right)$ and $D\left(e, e^{\prime}\right)=\left(d e, e-d e^{\prime}\right)$. The righthand complex is acyclic since $D\left(e, e^{\prime}\right)=0 \Rightarrow\left(e, e^{\prime}\right)=D\left(e^{\prime}, 0\right)$. The chain map $h:\left(\mathcal{B}^{*} \oplus \mathcal{E}^{*-1}, D\right) \rightarrow\left(\mathcal{B}^{*} \oplus \mathcal{C}^{*} \oplus \mathcal{C}^{*-1} \oplus \mathcal{E}^{*-1}, D^{\prime}\right)$, defined by $h(b, e)=(b, 0,0, e)$, where $D(b, e)=(d b, b-d e)$, is a quasi-isomorphism, since

$$
\begin{aligned}
0=D^{\prime}\left(b, c_{0}, c_{1}, e\right) & \Rightarrow \quad\left(b, c_{0}, c_{1}, e\right)=h\left(b, e+c_{1}\right)+D^{\prime}\left(0, c_{1}, 0,0\right) \\
h\left(b^{\prime}, e^{\prime}\right)=D^{\prime}\left(b, c_{0}, c_{1}, e\right) & \Rightarrow \quad\left(b^{\prime}, e^{\prime}\right)=D\left(b, e+c_{1}\right) .
\end{aligned}
$$

Let $k:\left(\mathcal{A}^{*} \oplus \mathcal{C}^{*-1}, D\right) \rightarrow\left(\mathcal{B}^{*} \oplus \mathcal{E}^{*-1}, D\right)$, defined by $k(a, c)=(a, c)$. If $D(a, c)=$ 0 , then $h(k(a, c))-f(a, c)=(0,-a,-c, c)=D^{\prime}(0,-c, 0,0)$. Therefore, $k$ is a quasi-isomorphism. Applying Theorem B and the definition of relative blown-up $\bar{p}$-intersection cohomology we get the result.

The following result comes directly from the Mayer-Vietoris sequence and the long exact sequence associated to a pair.

Proposition 12.5. Let $X$ be a compact filtered space. Let us consider the cone,

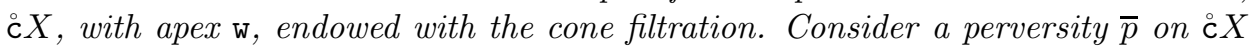
and denote also by $\bar{p}$ the induced perversity on $X$. Then

$$
\mathscr{H}_{\bar{p}}^{j}(\stackrel{\circ}{\mathrm{c}} X, \stackrel{\circ}{\mathrm{c}} X \backslash\{\mathrm{w}\} ; R)=\left\{\begin{array}{cc}
\mathscr{H}_{\bar{p}}^{j-1}(X ; R) & \text { if } j \geqslant \bar{p}(\mathrm{w})+2, \\
0 & \text { if } j \leqslant \bar{p}(\mathrm{w})+1 .
\end{array}\right.
$$

\section{Comparison between intersection cohomologies. Theorem $\mathbf{F}$}

If $R$ is a field, the existence of a quasi-isomorphism between the cochain complexes, $C_{D \bar{p}}^{*}(X ; R)$ and $\tilde{N}_{\bar{p}}^{*}(X ; R)$, has been established in [4, Theorem B] for GMperversities in the framework of filtered face sets. We generalize this result using general perversities without the condition $D \bar{p} \leqslant \bar{t}$. In order to eliminate this last constraint we use tame intersection cohomology and homology instead of intersection cohomology and homology.

Recall that a perverse CS set $(X, \bar{p})$ is locally $(D \bar{p}, R)$-torsion free if, for each singular stratum $S$ and each $x \in S$ with $\operatorname{link} L$, we have that $\mathfrak{T}_{\bar{p}(S)}^{D \bar{p}}(L ; R)=0$, i.e., the torsion $R$-submodule of $\mathfrak{H}_{\bar{p}(S)}^{D \bar{p}}(L ; R)$, vanishes (see [18]).

TheOrem F. Let $(X, \bar{p})$ be a paracompact separable perverse $C S$ set and $R$ a Dedekind ring. The blown-up and the tame intersection complexes, $\tilde{N}_{\bar{p}}^{*}(X ; R)$ and 
$\mathfrak{C}_{D \bar{p}}^{*}(X ; R)$, are related by a quasi-isomorphism if one of the following hypotheses is satisfied.

1) The ring $R$ is a field.

2) The space $X$ is a locally $(D \bar{p}, R)$-torsion free pseudomanifold.

Under any of these assumptions, there exists an isomorphism

$$
\mathscr{H}_{\bar{p}}^{*}(X ; R) \cong \mathfrak{H}_{D \bar{p}}^{*}(X ; R) .
$$

This isomorphism exists for the top perversity $\bar{t}$, for any CS set and any Dedekind ring (see [4, Remark 1.51]). This Theorem has a particular form in the classical setting of stratified pseudomanifolds and GM-perversities.

COROLlaRY 13.1. Let $\bar{p}$ be a GM-perversity, with complementary perversity $\bar{q}$, i.e., $\bar{q}(i)=i-2-\bar{p}(i)$ for $i \geqslant 2$. We consider a Dedekind ring $R$. Let $X$ be a paracompact separable classical pseudomanifold. The blown-up and the intersection complexes, $\tilde{N}_{\bar{p}}^{*}(X ; R)$ and $C_{\bar{q}}^{*}(X ; R)$, are related by a quasi-isomorphism if $X$ is locally $(\bar{q}, R)$-torsion free. So, we have an isomorphism $\mathscr{H}_{\bar{p}}^{*}(X ; R) \cong H_{\bar{q}}^{*}(X ; R)$.

The proof of Theorem $\mathrm{F}$, similar to that used in $\mathbf{5}$, Théorème $\mathrm{A}]$, meets the scheme used in [21, Theorem 10], 24, Lemma 1.4.1], [14, Section 5.1].

Proposition 13.2. Let $\mathcal{F}_{X}$ be the category whose objects are (stratified homeomorphic to) open subsets of a given paracompact and separable $C S$ set $X$ and whose morphisms are stratified homeomorphisms and inclusions. Let $\mathcal{A} b_{*}$ be the category of graded abelian groups. Let $F^{*}, G^{*}: \mathcal{F}_{X} \rightarrow \mathcal{A} b$ be two functors and $\Phi: F^{*} \rightarrow G^{*}$ a natural transformation satisfying the conditions listed below.

(i) $F^{*}$ and $G^{*}$ admit exact Mayer-Vietoris sequences and the natural transformation $\Phi$ induces a commutative diagram between these sequences,

(ii) If $\left\{U_{\alpha}\right\}$ is a disjoint collection of open subsets of $X$ and $\Phi: F_{*}\left(U_{\alpha}\right) \rightarrow G_{*}\left(U_{\alpha}\right)$ is an isomorphism for each $\alpha$, then $\Phi: F^{*}\left(\bigsqcup_{\alpha} U_{\alpha}\right) \rightarrow G^{*}\left(\bigsqcup_{\alpha} U_{\alpha}\right)$ is an isomorphism.

(iii) If $L$ is a compact filtered space such that $X$ has an open subset stratified homeomorphic to $\mathbb{R}^{i} \times \stackrel{\circ}{\mathrm{c}} L$ and, if $\Phi: F^{*}\left(\mathbb{R}^{i} \times\left({ }^{\circ} L \backslash\{\mathrm{v}\}\right)\right) \rightarrow G^{*}\left(\mathbb{R}^{i} \times\left({ }^{\circ} L \backslash\{\mathrm{v}\}\right)\right)$ is an isomorphism, then so is $\Phi: F^{*}\left(\mathbb{R}^{i} \times \stackrel{\circ}{\mathrm{c}} L\right) \rightarrow G^{*}\left(\mathbb{R}^{i} \times \stackrel{\circ}{\mathrm{c}} L\right)$. Here, $\mathrm{v}$ is the apex of the cone $\stackrel{\circ}{\mathrm{C}} L$.

(iv) If $U$ is an open subset of $X$ contained within a single stratum and homeomorphic to an Euclidean space, then $\Phi: F^{*}(U) \rightarrow G^{*}(U)$ is an isomorphism.

Then $\Phi: F^{*}(X) \rightarrow G^{*}(X)$ is an isomorphism.

Proof. Let $U$ be an open subset of $X$. We apply Lemma 13.3 taking for $P(U)$ the property

$$
\text { " } \Phi: F^{*}(U) \rightarrow G^{*}(U) \text { is an isomorphism." }
$$

Notice that the CS set $X$ is a separable, locally compact and metrizable space [5, Proposition 1.11]. We proceed by induction on the dimension of $X$, the result is immediate when $\operatorname{dim} X=0$, that is, a discrete space. The inductive step occurs in two stages.

- We first show $P(Y)$ for any open subset $Y$ of a fixed conical chart. We can suppose $Y=\mathbb{R}^{m} \times \stackrel{\circ}{\mathrm{c} L}$. If $L=\varnothing$, we apply (iv). Let us suppose $L \neq \varnothing$. We consider the basis $\mathcal{V}$ of open sets of $Y$, composed of 
- open subsets $V$ of $Y$, with $\mathrm{v} \notin V$, which are CS sets of strictly lower dimension than $X$,

- open subsets $V=B \times \stackrel{\circ}{\mathrm{c}}_{\varepsilon} L$, where $B \subset \mathbb{R}^{m}$ is an open $m$-cube, $\varepsilon>0$ and $\dot{\circ}_{\epsilon}=(L \times[0, \varepsilon[) /(L \times\{0\})$.

This family is closed by finite intersections and it verifies the assumptions of Lemma 13.3. For a), it suffices to apply the induction hypothesis together with (iii) and (iv). The property b) derives from (i) and property c) from (ii).

- We prove $P(X)$ by considering the open basis of $X$ consisting of open subsets of conical charts. This family is stable by finite intersections and verifies the assumptions of Lemma 13.3. The condition a) is a consequence of the first step. The two other conditions derive from (i) and (ii), as previously.

Lemma 13.3. Let $X$ be a locally compact topological space, metrizable and separable. We are given an open basis of $X, \mathcal{U}=\left\{U_{\alpha}\right\}$, closed by finite intersections, and a statement $P(U)$ on open subsets of $X$ satisfying the following three properties.

a) The property $P\left(U_{\alpha}\right)$ is true for all $\alpha$.

b) If $U, V$ are open subsets of $X$ for which properties $P(U), P(V)$ and $P(U \cap V)$ are true, then $P(U \cup V)$ is true.

c) If $\left(U_{i}\right)_{i \in I}$ is a family of open subsets of $X$, pairwise disjoint, verifying the property $P\left(U_{i}\right)$ for all $i \in I$, then $P\left(\bigsqcup_{i} U_{i}\right)$ is true.

Then the property $P(X)$ is true.

Proof. Since the family $\mathcal{U}$ is closed by finite intersections, the properties a) and b) imply that the property $P$ is true for any finite union of elements of $\mathcal{U}$.

Since the space $X$ is separable, metrizable and locally compact, then its Alexandroff's compactification $\hat{X}=X \sqcup\{\infty\}$ is metrizable (cf. [26. Exercise 23C]) and there exists a proper map, $f: X \rightarrow[0, \infty[$, defined by $f(x)=1 / d(\infty, x)$. We associate to $f$ a countable family of compact subsets, $A_{n}=f^{-1}([n, n+1])$. Each $A_{n}$ possesses a finite cover consisting of open subsets $U_{\alpha} \in \mathcal{U}$, included in $f^{-1}(] n-1 / 2, n+3 / 2[)$; we denote by $U_{n}$ the union of the elements of this cover. Since the open subset $U_{n}$ is a finite union of elements of $\mathcal{U}$, then the property $P\left(U_{n}\right)$ is true.

Let $U_{\text {even }}=\bigsqcup_{n} U_{2 n}$ and let $U_{\text {odd }}=\bigsqcup_{n} U_{2 n+1}$. The hypothesis c) implies that $P\left(U_{\text {even }}\right)$ and $P\left(U_{\text {odd }}\right)$ are true. Furthermore, since the intersection $U_{2 n} \cap U_{2 n+1}$ is a finite union of elements $U_{\alpha}$ of $\mathcal{U}$, then the property $P\left(U_{2 n} \cap U_{2 n+1}\right)$ is true. From $U_{\text {even }} \cap U_{\text {odd }}=\bigsqcup_{n} U_{2 n} \cap U_{2 n+1}$ and from the hypothesis c), we deduce that the property $P\left(U_{\text {even }} \cup U_{\text {odd }}\right)$ is true. The conclusion then follows from $U_{\text {even }} \cup U_{\text {odd }}=$ $\bigcup_{n} U_{n} \supset \bigcup_{n} A_{n}=X$.

Let $X$ be a filtered space. We construct the map

$$
\chi: \tilde{N}^{*}(X ; R) \rightarrow \mathfrak{C}^{*}(X ; R)
$$

as follows. If $\omega \in \tilde{N}^{*}(X ; R)$ and if $\sigma: \Delta=\Delta_{0} * \cdots * \Delta_{n} \rightarrow X$ is a regular simplex, we set:

$$
\chi(\omega)(\sigma)=\omega_{\sigma}(\widetilde{\Delta}) .
$$

Proposition 13.4. Let $(X, \bar{p})$ be a perverse space. The operator $\chi$ induces a chain map, $\chi: \widetilde{N}_{\bar{p}}^{*}(X ; R) \rightarrow \mathfrak{C}_{D \bar{p}}^{*}(X ; R)$. 
Proof. Recall that $\mathfrak{C}_{D \bar{p}}^{*}(X ; R)=\operatorname{Hom}\left(\mathfrak{C}_{*}^{D \bar{p}}(X ; R), R\right)$. Since the simplices coming from $\mathfrak{C}_{*}^{\bar{D}}(X ; R)$ are regular, then the definition of $\chi$ makes sense. This is the reason of considering tame intersection chains instead of intersection chains.

We need to prove $\chi \circ \delta=\mathfrak{d}^{*} \circ \chi$, where $\mathfrak{d}^{*}$ is the linear dual of $\mathfrak{d}$ (see Definition [5.4). Consider a cochain $\omega$ of $\tilde{N}_{\bar{p}}^{*}(X ; R)$ and a chain $\xi \in \mathfrak{C}_{*}^{D \bar{p}}(X ; R)$. We prove $\chi(\delta \omega)(\xi)=\left(\mathfrak{d}^{*} \chi\right)(\omega)(\xi)=-(-1)^{|\omega|} \chi(\omega)(\mathfrak{d} \xi)$. In fact, it suffices to prove $\chi(\delta \omega)(\sigma)=-(-1)^{|\omega|} \chi(\omega)(\mathfrak{d} \sigma)$ for a regular simplex $\sigma: \Delta \rightarrow X D \bar{p}$-allowable. Let us suppose that:

$$
\omega_{\sigma}\left(\mathcal{H}_{i}\right)=0 \text { for each hidden face } \mathcal{H}_{i}
$$

$($ see $(6.5))$. Then

$\chi(\delta \omega)(\sigma)=(\delta \omega)_{\sigma}(\widetilde{\Delta})=-(-1)^{|\omega|} \omega_{\sigma}(\partial \widetilde{\Delta})=-(-1)^{|\omega|} \omega_{\sigma}(\widetilde{\mathfrak{d} \Delta})=-(-1)^{|\omega|} \chi(\omega)(\mathfrak{d} \sigma)$.

Let us prove the claim (13.1). We suppose $\omega_{\sigma}\left(\mathcal{H}_{i}\right) \neq 0$ for some hidden face $\mathcal{H}_{i}$. By definition of a hidden face, notice that $\Delta_{n-i} \neq \varnothing$, thus there exists an $i$ dimensional stratum $S$ of $X$ with $S \cap \operatorname{Im} \sigma \neq \varnothing$. Then, since $\sigma$ is $D \bar{p}$-allowable and $\omega$ is $\bar{p}$-allowable,

- $0 \leqslant|\Delta|_{\leqslant n-i}=\|\sigma\|_{S} \leqslant \operatorname{dim} \sigma-\operatorname{codim} S+D \bar{p}(S)=\operatorname{dim} \sigma-\bar{p}(S)-2$, and

- $0 \leqslant|\Delta|_{>n-i}=\left|\mathcal{H}_{i}\right|_{>n-i}=\left\|\mathbf{1}_{\mathcal{H}_{i}}\right\|_{\operatorname{codim} S} \leqslant(1)\left\|\omega_{\sigma}\right\|_{\operatorname{codim} S} \leqslant \bar{p}(S)$,

where $\leqslant_{(1)}$ comes from $\omega_{\sigma}\left(\mathcal{H}_{i}\right) \neq 0$. We conclude that $\bar{p}(S)$ is finite. Adding up these two inequalities, we get $\operatorname{dim} \sigma-1=\operatorname{dim} \Delta-1=|\Delta|_{\leqslant n-i}+|\Delta|_{>n-i} \leqslant$ $\left\|\omega_{\sigma}\right\|_{S}+\|\sigma\|_{S} \leqslant \operatorname{dim} \sigma-2$. This contradiction gives the claim (13.1) and ends the proof.

Proof of Theorem E. By hypothesis, $X$ is a separable paracompact CS set. Properties (i), (ii), (iv) of Proposition 13.2 are verified. For the item (iii), we consider a filtered compact space $L$, for which the chain map of Proposition 13.4induces an isomorphism $\chi^{*}: \mathscr{H}_{\bar{p}}^{*}\left(\mathbb{R}^{i} \times L \times\right] 0, \infty[; R) \stackrel{\cong}{\rightrightarrows} \mathfrak{H}_{\bar{q}}^{*}\left(\mathbb{R}^{i} \times L \times\right] 0, \infty[; R)$. Since the space $\left.\mathbb{R}^{i} \times L \times\right] 0, \infty$ [ is an open subset of $\mathbb{R}^{i} \times \stackrel{\circ}{\mathrm{c}} L$, the following diagram commutes.

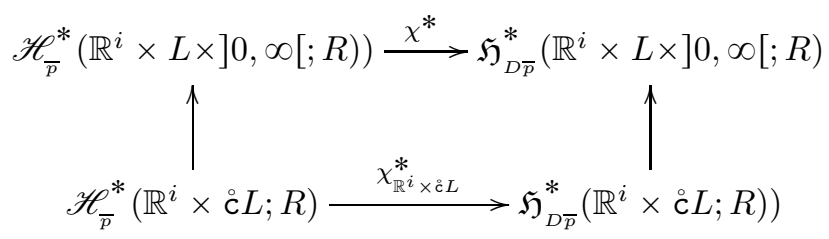

Recall the blown-up intersection cohomology computation (see Theorem D and Theorem E):

$$
\begin{aligned}
\mathscr{H}_{\bar{p}}^{k}\left(\mathbb{R}^{i} \times \stackrel{\circ}{\mathrm{c}} L ; R\right) & =\mathscr{H}_{\bar{p}}^{k}(\stackrel{\circ}{\mathrm{c}} L ; R) \\
& =\left\{\begin{array}{cl}
\mathscr{H}_{\bar{p}}^{k}(L ; R)=\mathscr{H}_{\bar{p}}^{k}\left(\mathbb{R}^{i} \times L \times\right] 0, \infty[; R) & \text { if } k \leqslant \bar{p}(\mathrm{w}), \\
0 & \text { if } k>\bar{p}(\mathrm{w}) .
\end{array}\right.
\end{aligned}
$$

The tame intersection cohomology is determined in [14, Chapter 7]: 


$$
\begin{aligned}
\mathfrak{H}_{D \bar{p}}^{k}\left(\mathbb{R}^{i} \times \stackrel{\circ}{\mathrm{C}} L ; R\right) & =\mathfrak{H}_{D \bar{p}}^{k}(\stackrel{\circ}{\mathrm{c}} L ; R) \\
& =\left\{\begin{array}{cll}
\mathfrak{H}_{D \bar{p}}^{k}(L ; R)=\mathfrak{H}_{D \overline{\bar{p}}}^{k}\left(\mathbb{R}^{i} \times L \times\right] 0, \infty[; R) & \text { if } & k \leqslant \bar{p}(\mathrm{w}), \\
\operatorname{Ext}\left(\mathfrak{H}_{k-1}^{D \bar{p}}(L ; R), R\right) & \text { if } & k=\bar{p}(\mathrm{w})+1, \\
0 & \text { if } & k>\bar{p}(\mathrm{w})+1 .
\end{array}\right.
\end{aligned}
$$

The involved stratifications on $L$ are different in these computations but the cohomologies are the same (see subsections 3.1, 5.1).

In case 1$)$ we have $\operatorname{Ext}\left(\mathfrak{H}_{\bar{p}(w)}^{D \bar{p}}(L ; R), R\right)=0$ since $R$ is a field. In case 2$)$ the link $L$ is a compact pseudomanifold. According to [14, Section 5.7], the tame intersection homology of $L$ is finitely generated. Since the torsion module of $\mathfrak{H}_{\bar{p}(\mathbf{w})}^{D \bar{P}}(L ; R)$ is zero by hypothesis, we deduce $\operatorname{Ext}\left(\mathfrak{H}_{\bar{p}(w)}^{D \bar{p}}(L ; R), R\right)=0$.

So, in both cases, the vertical maps of (13.2) are isomorphisms. Since $\chi^{*}$ is an isomorphism then $\chi_{\mathbb{R}^{i} \times \dot{ }}^{*} L$ is also an isomorphism.

For some particular perversities the blown-up intersection cohomology is described in terms of the ordinary cohomology, denoted by $H^{*}(-; R)$.

Proposition 13.5. Let $X$ be a paracompact separable CS set. Then

(a) $\mathscr{H}_{0}^{*}(X ; R) \cong H^{*}(X ; R)$ if $X$ is normal.

(b) $\mathscr{H}_{\bar{p}}^{*}(X ; R) \cong H^{*}(X, \Sigma ; R)$ if $\bar{p}<0$.

(c) $\mathscr{H}_{\bar{p}}^{*}(X ; R) \cong H^{*}(X \backslash \Sigma ; R)$ if $\bar{p}>\bar{t}$.

These isomorphisms preserve the cup product.

Proof. The complex of singular chains (resp. relative chains) with coefficients in $R$, is denoted by $S_{*}(X ; R)$ (resp. $S_{*}(X, \Sigma ; R)$ ). It computes the ordinary homology $H_{*}(X ; R)\left(\operatorname{resp} . H_{*}(X, \Sigma ; R)\right)$.

(a) Let $F: S^{*}(X ; R) \rightarrow \tilde{N}_{\bar{\sigma}}^{*}(X ; R)$ be the differential operator defined by $F(\omega)_{\sigma}=\mu^{*}\left(\sigma^{*}(\omega)\right)$, for any regular filtered simplex $\sigma: \Delta \rightarrow X$. The operator $\mu^{*}$ is associated to the $\mu$-amalgamation of $\Delta$ (see 8.1.1). Applying Proposition 7.5 repeatedly, we conclude that $F$ is a chain map commuting with the cup product. By definition of $\mu^{*}$ we have $\left\|F(\omega)_{\sigma}\right\|_{\ell} \leqslant\left\|\sigma^{*}(\omega)\right\|_{0}=0$. The operator $F$ is therefore well defined.

We consider the statement: "The operator $F$ is a quasi-isomorphism" and we prove it by using Proposition 13.2. We verify the four properties (i)-(iv) of this Proposition.

(i) It is well known that the functor $S^{*}(-; R)$ verifies the Mayer-Vietoris property. It has been proven in Theorem $\mathrm{C}$ that the functor $\widetilde{N}_{\overline{0}}^{*}(-; R)$ also verifies Mayer-Vietoris property. One easily checks that $F$ induces a commutative diagram between these sequences.

(ii),(iv) Straightforward.

(iii) It is well known that $H^{*}\left(\mathbb{R}^{i} \times \stackrel{\circ}{\mathrm{c}} L ; R\right)=H^{0}\left(\mathbb{R}^{i} \times \stackrel{\circ}{\mathrm{c}} L ; R\right)=R$ (constant cochains). From Theorems D, E we have $\mathscr{H}_{\overline{0}}^{*}\left(\mathbb{R}^{i} \times \stackrel{\circ}{\mathrm{c}} L ; R\right)=\mathscr{H}_{\overline{0}}^{0}(L ; R)$. Let us consider a basis point $x_{0} \in L \backslash \Sigma$, which is connected from [14, Lemma 2.6.3]. Also, for any point $x_{1} \in L$ there exists a regular simplex 
$\sigma:[0,1] \rightarrow L$ going from $x_{1}$ to $x_{0}$. If $\omega \in \widetilde{N}_{\overline{0}}^{0}(L ; R)$ is a cocycle, the cochain $\omega_{\sigma}$ takes the same value on $x_{1}$ and on $x_{0}$. This implies $\mathscr{H}_{\overline{0}}^{0}(L ; R)=R$.

(b) Consider the previous operator $F: S^{*}(X ; R) \rightarrow \tilde{N}^{*}(X ; R)$ defined by $F(\omega)_{\sigma}=\mu^{*}\left(\sigma^{*}(\omega)\right)$, for any regular filtered simplex $\sigma: \Delta \rightarrow X$. We know that it is a chain map commuting with the cup product. If $\omega$ vanishes on $\Sigma$ then $\left\|F(\omega)_{\sigma}\right\|_{\ell}=-\infty$ for any $\ell \in\{1, \ldots, n\}$. So, $F: S^{*}(X, \Sigma ; R) \rightarrow \widetilde{N}_{\bar{p}}^{*}(X ; R)$ is well defined. We consider the statement: "The operator $F$ is a quasi-isomorphism" and we prove it as in the previous case. In fact, the only item to prove is (iii). It comes from $H^{*}\left(\mathbb{R}^{i} \times \stackrel{\circ}{\mathrm{c}} L, \mathbb{R}^{i} \times \stackrel{\circ}{\mathrm{c}} \Sigma_{L} ; R\right)=0=\mathscr{H}_{\bar{p}}^{*}\left(\mathbb{R}^{i} \times \stackrel{\circ}{\mathrm{c}} L ; R\right)$, induced by Theorems D, E

(c) Consider the natural restriction $\gamma: \widetilde{N}_{\bar{p}}^{*}(X ; R) \rightarrow \widetilde{N}_{\bar{p}}^{*}(X \backslash \Sigma ; R)=S^{*}(X \backslash \Sigma ; R)$. It is a chain map commuting with the cup product. We consider the statement: "The operator $\gamma$ is a quasi-isomorphim" we proceed as in the previous case. In fact, the only property to prove is the item (iii) of Proposition 13.2. We know that $H^{*}\left(\mathbb{R}^{i} \times \stackrel{\circ}{\mathrm{c}} L \backslash \Sigma_{\mathbb{R}^{i} \times \mathrm{c} L} ; R\right)=H^{*}\left(\mathbb{R}^{i} \times\left(L \backslash \Sigma_{L}\right) \times\right] 0, \infty[; R)=H^{*}\left(L \backslash \Sigma_{L} ; R\right)=$ $H^{*}\left(\mathbb{R}^{i} \times(\stackrel{\circ}{\mathrm{c}} L \backslash\{\mathrm{v}\}) \backslash \Sigma_{\mathbb{R}^{i} \times\left({ }^{\circ} L \backslash\{\mathrm{v}\}\right)} ; R\right)$. On the other hand, we have $\mathscr{H}_{\bar{p}}^{*}\left(\mathbb{R}^{i} \times \stackrel{\circ}{\mathrm{c}} L ; R\right)=$ $\mathscr{H}_{\bar{p}}^{*}(L ; R)=\mathscr{H}_{\bar{p}}^{*}\left(\mathbb{R}^{i} \times(\stackrel{\circ}{\mathrm{c}} L \backslash\{\mathrm{v}\}) ; R\right)$ (cf. Theorems D] E). It suffices to apply the hypothesis of (iii).

\section{Topological invariance. Theorem G,}

We prove in this section that the blown-up intersection cohomology is a topological invariant, working with CS sets and GM-perversities. We follow the procedure of King [21] (see also [14]).

14.1. Intrinsic filtration. A key ingredient in the King's proof of the topological invariance is the intrinsic filtration of a CS set $X$. It was introduced in [21, crediting to Sullivan, see also [20]. We refer the reader to [14] where there is an exhaustive study of this notion.

Let $X$ be an $n$-dimensional filtered space. Two points $x, y \in X$ are equivalent if there exists a homeomorphism $h:(U, x) \rightarrow(V, y)$, where $U, V$ are neighborhoods of $x, y$ respectively. The local structure of a CS set implies that two points of the same stratum are necessarily equivalent. Hence the equivalence classes are unions of components of strata of X.

Let $Y_{i}$ be the union of the equivalence classes which only contain components of strata of dimension $\leqslant i$. The filtration $Y_{0} \subset Y_{1} \subset \cdots \subset Y_{n}=X$ is the intrinsic filtration of $X$, denoted by $X^{*}$. It is proved that $X^{*}$ is an $n$-dimensional CS set 21. Since the filtration $X^{*}$ coarsens the original filtration of $X$ then the identity map $\nu: X \rightarrow X^{*}$ is a stratified map, called the intrinsic aggregation (see Definition 1.5). Recall that, given a perversity $\bar{p}$, the map $\nu$ induces a morphism $\nu^{*}: \mathscr{H}_{\bar{p}}^{*}\left(X^{*} ; R\right) \rightarrow \mathscr{H}_{\bar{p}}^{*}(X ; R)$ (see Theorem $\mathrm{A}$ ).

Proposition 14.1. Let $\bar{p}$ be a GM-perversity. Let $X$ be a paracompact CS set with no codimension one strata. Let $S$ be a stratum of $X$ and $(U, \varphi)$ a conical chart of a point $x \in S$. If the intrinsic aggregation $\nu: X \rightarrow X^{*}$ induces the isomorphism $\nu_{*}: \mathscr{H}_{\bar{p}}^{*}\left((U \backslash S)^{*} ; R\right) \stackrel{\cong}{\rightrightarrows} \mathscr{H}_{\bar{p}}^{*}(U \backslash S ; R)$ then it also induces the isomorphism,

$$
\nu_{*}: \mathscr{H}_{\bar{p}}^{*}\left(U^{*} ; R\right) \stackrel{\cong}{\longrightarrow} \mathscr{H}_{\bar{p}}^{*}(U ; R) \text {. }
$$


Proof. We analyze the local structure of $X$ and $X^{*}$. Without loss of generality, we can suppose $U=\mathbb{R}^{k} \times \stackrel{\circ}{\mathrm{C}} W$, where $W$ is a compact filtered space (possibly empty) and $S \cap U=\mathbb{R}^{k} \times\{\mathrm{w}\}$, w being the apex of the cone $\stackrel{\circ}{\mathrm{c}} W$. Following [21, Lemma 2 and Proposition 1], there exists a homeomorphism

$$
h:\left(\mathbb{R}^{k} \times \stackrel{\circ}{\mathrm{c} W}\right)^{*} \cong \mathbb{R}^{m} \times \stackrel{\circ}{\cong} L,
$$

which is also a stratified map, where $L$ is a compact filtered space (possibly empty) and $m \geqslant k$. Moreover, $h$ verifies

$$
h\left(\mathbb{R}^{k} \times\{\mathrm{w}\}\right) \subset \mathbb{R}^{m} \times\{\mathrm{v}\} \text { and } h^{-1}\left(\mathbb{R}^{m} \times\{\mathrm{v}\}\right)=\mathbb{R}^{k} \times \stackrel{\circ}{ } A,
$$

where $A$ is an $(m-k-1)$ sphere and $\mathrm{v}$ is the apex of the cone ${ }^{\circ} L$. Now, the hypothesis and the conclusion of the Proposition become

$$
h: \mathscr{H}_{\bar{p}}^{*}\left(\mathbb{R}^{m} \times \stackrel{\circ}{c} L \backslash h\left(\mathbb{R}^{k} \times\{\mathrm{w}\}\right) ; R\right) \stackrel{\cong}{\rightrightarrows} \mathscr{H}_{\bar{p}}^{*}\left(\mathbb{R}^{k} \times \stackrel{\circ}{c} W \backslash\left(\mathbb{R}^{k} \times\{\mathrm{w}\}\right) ; R\right)
$$

and

$$
h: \mathscr{H}_{\bar{p}}^{*}\left(\mathbb{R}^{m} \times \stackrel{\circ}{\mathrm{c}} L ; R\right) \stackrel{\cong}{\rightrightarrows} \mathscr{H}_{\bar{p}}^{*}\left(\mathbb{R}^{k} \times \stackrel{\circ}{\mathrm{c} W} ; R\right) .
$$

The existence of the homeomorphism (14.1) implies $k+s=m+t$, and $s \geqslant t$, since $m \geqslant k$, where $s=\operatorname{dim} W$ and $t=\operatorname{dim} L$.

The result is clear when $s=-1$. So, we can suppose $s \geqslant 0$ which implies that the stratum $\mathbb{R}^{k} \times\{\mathrm{w}\}$ is singular. Since $X$ has no codimension one strata, we indeed have $s \geqslant 1$. When $t=-1$ then $L=\varnothing, s=\operatorname{dim} A$ and therefore

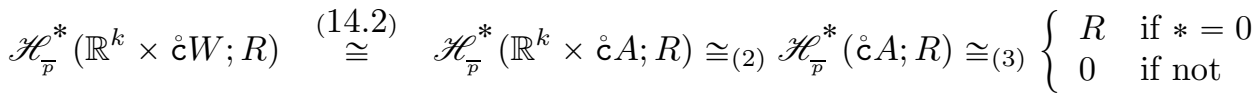

$$
\begin{aligned}
& \cong \mathscr{H}_{\bar{p}}^{*}\left(\mathbb{R}^{m} \times \stackrel{\circ}{\mathrm{c}} L ; R\right) \text {. }
\end{aligned}
$$

Here, $\cong_{(2)}$ is Theorem $\mathrm{D}$ and $\cong_{(3)}$ comes from

- Theorem E and

- $0 \leqslant \bar{p}(s+1) \leqslant \bar{t}(s+1)=s-1<\operatorname{dim} A$ since $\bar{p}$ is a GM-perversity and $s \geqslant 1$.

So, we can suppose $t \geqslant 0$ which implies that the stratum $\mathbb{R}^{m} \times\{\mathrm{v}\}$ is singular.

We establish (14.4) in two different cases.

- First case: $i>\bar{p}(s+1)$.

Theorem E gives $\mathscr{H}_{\bar{p}}^{i}\left(\mathbb{R}^{k} \times \stackrel{\complement}{c} W ; R\right)=0$ and then we have to prove that $\mathscr{H}_{\bar{p}}^{i}\left(\mathbb{R}^{m} \times \stackrel{\circ}{\mathrm{c}} L ; R\right)=0$. Since the stratum $\mathbb{R}^{m} \times\{\mathrm{v}\}$ is singular and $\bar{p}$ is a GMperversity then $i>\bar{p}(s+1) \geqslant \bar{p}(t+1)$. Now, TheoremEapplied to $\mathscr{H}_{\bar{p}}^{i}\left(\mathbb{R}^{m} \times \stackrel{\circ}{\mathrm{c}} L ; R\right)$ gives the result.

- Second case : $i \leqslant \bar{p}(s+1)$. We have the isomorphisms

$$
\begin{array}{r}
(14.5) \mathscr{H}_{\bar{p}}^{i}\left(\mathbb{R}^{m} \times \stackrel{\circ}{\mathrm{c}} L \backslash h\left(\mathbb{R}^{k} \times\{\mathrm{w}\}\right) ; R\right) \cong(1) \mathscr{H}_{\bar{p}}^{i}\left(\mathbb{R}^{k} \times \stackrel{\mathrm{c}}{ } W \backslash\left(\mathbb{R}^{k} \times\{\mathrm{w}\}\right) ; R\right) \cong \\
\mathscr{H}_{\bar{p}}^{i}\left(\mathbb{R}^{k} \times(\stackrel{\circ}{\mathrm{c}} W \backslash\{\mathrm{w}\}) ; R\right) \cong \mathscr{H}_{\bar{p}}^{i}\left(\mathbb{R}^{k} \times\right] 0,1[\times W ; R) \cong(2) \mathscr{H}_{\bar{p}}^{i}(W ; R),
\end{array}
$$


where $\cong_{(1)}$ is the hypothesis (14.3) and $\cong_{(2)}$ is Theorem D. Write $h\left(\mathbb{R}^{k} \times\{\mathrm{w}\}\right)=$ $B \times\{\mathrm{v}\} \subset \mathbb{R}^{m} \times\{\mathrm{v}\}$, with $B$ closed. We obtain a new sequence of isomorphisms

$$
\begin{array}{r}
(14.6) \quad \mathscr{H}_{\bar{p}}^{i}\left(\mathbb{R}^{m} \times \stackrel{\circ}{\mathrm{c}} L \backslash h\left(\mathbb{R}^{k} \times\{\mathrm{w}\}\right), \mathbb{R}^{m} \times \stackrel{\circ}{\mathrm{c}} L \backslash \mathbb{R}^{m} \times\{\mathrm{v}\} ; R\right) \cong \\
\mathscr{H}_{\bar{p}}^{i}\left(\left(\mathbb{R}^{m} \times \stackrel{\circ}{\mathrm{c}} L\right) \backslash(B \times\{\mathrm{v}\}), \mathbb{R}^{m} \times(\stackrel{\circ}{\mathrm{c}} L \backslash\{\mathrm{v}\}) ; R\right) \cong(1) \\
\mathscr{H}_{\bar{p}}^{i}\left(\left(\mathbb{R}^{m} \backslash B\right) \times \stackrel{\circ}{\mathrm{c}} L,\left(\mathbb{R}^{m} \backslash B\right) \times(\stackrel{\circ}{\mathrm{c}} L \backslash\{\mathrm{v}\}) ; R\right) \cong \mathscr{H}_{\bar{p}}^{i}\left(\left(\mathbb{R}^{m} \backslash B\right) \times(\stackrel{\circ}{\mathrm{c}} L, \stackrel{\circ}{\mathrm{c}} L \backslash\{\mathrm{v}\}) ; R\right) \cong(2) \\
\left.\mathscr{H}_{\bar{p}}^{i} \mathbb{R}^{k+1} \times A \times(\stackrel{\circ}{\mathrm{c}} L, \stackrel{\circ}{\mathrm{c}} L \backslash\{\mathrm{v}\}) ; R\right) \cong(3) \\
\mathscr{H}_{\bar{p}}^{i}(\stackrel{\circ}{\mathrm{c}} L, \stackrel{\circ}{\mathrm{c}} L \backslash\{\mathrm{v}\} ; R) \oplus \mathscr{H}_{\bar{p}}^{i-m+1+k}(\stackrel{\circ}{\mathrm{c}} L, \stackrel{\circ}{\mathrm{c}} L \backslash\{\mathrm{v}\} ; R) .
\end{array}
$$

The isomorphism $\cong_{(1)}$ is the excision of $B \times\left({ }^{\circ} L \backslash\{\mathrm{v}\}\right)$ (see Proposition 12.4), $\cong_{(2)}$ comes from (14.2) and $\cong_{(3)}$ from Theorem $\mathrm{D}$ and Proposition 11.4. Notice that $\mathbb{R}^{m} \times \stackrel{\circ}{c} L$, $\stackrel{\circ}{\mathrm{c}} L$ and $\stackrel{\circ}{\mathrm{c}} L \backslash\{\mathrm{w}\}$ are $F_{\sigma}$-subsets, [26. Exercise $\left.3 \mathrm{H}\right]$, of $X^{*}$ and therefore paracompact spaces [26. Theorem $20.12 \mathrm{a}), \mathrm{b})]$. The hypothesis on $i$ implies $i-m+1+k \leqslant \bar{p}(s+1)-s+1+t \leqslant \bar{p}(t+1)+1$, since $\bar{p}$ is a GM-perversity. Then $\mathscr{H}_{\bar{p}}^{i-m+1+k}(\stackrel{\circ}{\mathrm{c}} L, \stackrel{\circ}{\mathrm{c}} L \backslash\{\mathrm{v}\} ; R)$ vanishes (see Proposition 12.5). We have proved that $\mathscr{H}_{\bar{p}}^{i}\left(\mathbb{R}^{m} \times \stackrel{\circ}{\mathrm{c}} L \backslash h\left(\mathbb{R}^{k} \times\{\mathrm{w}\}\right), \mathbb{R}^{m} \times \stackrel{\circ}{\mathrm{c}} L \backslash \mathbb{R}^{m} \times\{\mathrm{v}\} ; R\right) \cong \mathscr{H}_{\bar{p}}^{i}(\stackrel{\circ}{\mathrm{c}} L, \stackrel{\circ}{\mathrm{c}} L \backslash\{\mathrm{v}\} ; R)$. Finally, from this isomorphism, from the long exact sequence of a pair (12.9) and from (14.5), we get that $\mathscr{H}_{\bar{p}}^{i}(W ; R) \cong \mathscr{H}_{\bar{p}}^{i}(\stackrel{\circ}{\mathrm{c}} L ; R)$. Applying Theorem $\mathrm{E}$ and Theorem $\mathrm{D}$ we have $\mathscr{H}_{\bar{p}}^{i}\left(\mathbb{R}^{m} \times \stackrel{\circ}{\mathrm{c}} L ; R\right) \cong \mathscr{H}_{\bar{p}}^{i}(\mathrm{c} L ; R) \cong \mathscr{H}_{\bar{p}}^{i}(W ; R) \cong \mathscr{H}_{\bar{p}}^{i}(\stackrel{\circ}{\mathrm{c}} W ; R) \cong$ $\mathscr{H}_{\bar{p}}^{i}\left(\mathbb{R}^{k} \times \stackrel{\circ}{c} W ; R\right)$.

Theorem G. Suppose $X$ is a separable paracompact $C S$ set with no codimension one strata. Let $\bar{p}$ be a GM-perversity. Then, the intrinsic aggregation $\nu: X \rightarrow X^{*}$ induces an isomorphism $\mathscr{H}_{\bar{p}}^{*}(X ; R) \cong \mathscr{H}_{\bar{p}}^{*}\left(X^{*} ; R\right)$. It follows that $\mathscr{H}_{\bar{p}}^{*}(X ; R)$ is independent (up to isomorphism) of the choice of a stratification of $X$ as $C S$ set with no codimension one strata.

In particular, if $X^{\prime}$ is another CS set with no codimension one strata which is homeomorphic to $X$ (not necessarily stratified homeomorphic), then $\mathscr{H}_{\bar{p}}^{*}(X ; R) \cong$ $\mathscr{H}_{\bar{p}}^{*}\left(X^{\prime} ; R\right)$. These $\bar{p}$-depending isomorphisms can be chosen to preserve the cup product.

Proof. We consider the statement: "The intrinsic aggregation $\nu: X \rightarrow X^{*}$ induces a quasi-isomorphism" and we prove it by using Proposition 13.2. We verify the four conditions of this Proposition.

(i) The functor $\tilde{N}_{\bar{p}}^{*}(-; R)$ satisfies Mayer-Vietoris property (see TheoremC). Notice that we know from [26. Theorem $20.12 \mathrm{~b}$ )] that $X^{*}$ is paracompact.

(ii),(iv) Straightforward.

(iii) See Proposition 14.1

Last statement comes from the fact that $\nu$ is a stratified map and Theorem A.

\section{Decomposition of the ordinary cap product.}

In this last section, we show how the ordinary cap product factorizes through the cap product we have defined for the blown-up intersection cohomology. This result extends the factorization developed in [7, Theorem A]. 
Proposition 15.1. Let $X$ be a normal separable paracompact $C S$ set endowed with two perversities $\bar{p}, \bar{p}$ verifying $\overline{0} \leqslant \bar{p}$ and $\bar{p}+\bar{p} \leqslant \bar{t}$. Then, there exists a commutative diagram

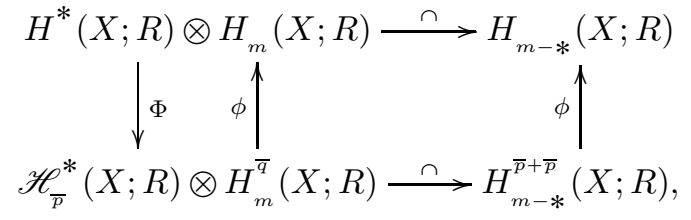

that is, $\phi(\Phi(\alpha) \cap \xi)=\alpha \cap \phi(\xi)$, for each $\alpha \in H^{*}(X ; R)$ and $\xi \in H_{m}^{\bar{q}}(X ; R)$. The map $\Phi$ preserves the $\cup$-product. Moreover, if $(\bar{p}, \bar{p})=(\overline{0}, \bar{t})$ then $\Phi$ and $\phi$ can be chosen isomorphisms.

Proof. Consider the natural inclusions $\tilde{N}_{\bar{\sigma}}^{*}(X ; R) \hookrightarrow \tilde{N}_{\bar{p}}^{*}(X ; R), C_{*}^{\bar{q}}(X ; R) \hookrightarrow$ $C_{*}^{\bar{t}}(X ; R)$ and $C_{*}^{\bar{p}+\bar{q}}(X ; R) \hookrightarrow C_{*}^{\bar{t}}(X ; R)$. So, it suffices to prove the statement for $(\bar{p}, \bar{q})=(\overline{0}, \bar{t})($ see Remark 6.8) $)$.

The complex of singular chains (resp. cochains), with coefficients on $R$, is denoted by $S_{*}(X ; R)\left(\right.$ resp. $\left.S^{*}(X ; R)\right)$, it computes the ordinary homology $H_{*}(X ; R)$ (resp. ordinary cohomology $H^{*}(X ; R)$ ). We proceed in three steps.

- Construction of $\phi$. We consider the inclusion $\iota: C_{*}^{\bar{t}}(X ; R) \hookrightarrow S_{*}(X ; R)$. We prove the statement : "The map $\iota$ is a quasi-isomorphim" by using [14, Theorem 5.1.4]. Let us verify the four conditions (1)-(4) of this Theorem.

(1) It is well known that the functor $S_{*}(-; R)$ verifies the Mayer-Vietoris property. It has been proven in [5, Proposition 4.1] that the functor $C_{*}^{\bar{t}}(-; R)$ also verifies Mayer-Vietoris property.

(2) Since the support of chains are compact.

(3) Since

$$
\begin{aligned}
& H_{*}\left(\mathbb{R}^{i} \times \stackrel{\circ}{\mathrm{c}} L ; R\right)=H_{0}\left(\mathbb{R}^{i} \times \stackrel{\circ}{\mathrm{c}} L ; R\right)=R \stackrel{\text { Normal }}{=} H_{0}(L ; R) \\
& =H_{0}\left(\mathbb{R}^{i} \times(\stackrel{\circ}{\mathrm{c}} L \backslash\{\mathrm{v}\}) ; R\right) \\
& \text { Hyp. (3) } H_{0}^{\bar{t}}\left(\mathbb{R}^{i} \times(\stackrel{\circ}{=} L \backslash\{\mathrm{v}\}) ; R\right) \stackrel{[\text { [ Prop. }}{=}{ }^{5.4]} H_{*}^{\bar{t}}\left(\mathbb{R}^{i} \times \stackrel{\circ}{\mathrm{c}} L ; R\right)
\end{aligned}
$$

(4) Straightforward.

The differential operator $\phi$ is the isomorphism $\iota_{*}$.

- Construction of $\Phi$. Consider the operator $F$ constructed in the proof of Proposition 13.5 (a) and set $\Phi=F_{*}$.

- Diagram commutativity. This is a local question. We can consider a cochain $\omega \in \tilde{N}^{*}(X)$ and a regular simplex $\sigma: \Delta \rightarrow X$. We have: $\phi(\Phi(\omega) \cap$ $\sigma)=\sigma_{*} \mu_{*}\left((\Phi(\omega))_{\sigma} \widetilde{\cap} \widetilde{\Delta}\right)=\sigma_{*} \mu_{*}\left(\mu^{*}\left(\sigma^{*} \omega\right) \widetilde{\cap} \widetilde{\Delta}\right)^{\operatorname{Prop} \underline{\underline{\underline{7.5}}}(2)} \sigma_{*}\left(\sigma^{*} \omega \cap\right.$ $\Delta)=\omega \cap \sigma=\omega \cap \phi(\sigma)$.

The case $\bar{q}=\overline{0}$ corresponds to the decomposition of [7, Theorem A]. If we work with non positive perversities, we have a decomposition of the cap product as follows. 
Proposition 15.2. Let $X$ be a separable paracompact $C S$ set endowed with two perversities $\bar{p}<\overline{0}$ and $\bar{q}<\overline{0}$. Then, there exists a commutative diagram

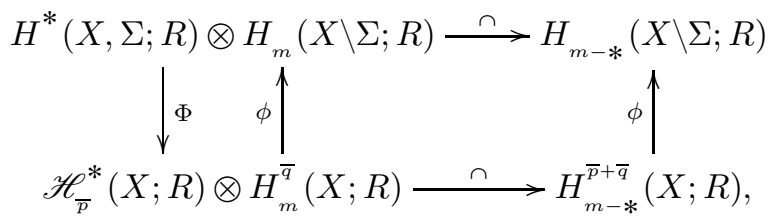

that is, $\phi(\Phi(\alpha) \cap \xi)=\alpha \cap \phi(\xi)$, for each $\alpha \in H^{*}(X, \Sigma ; R)$ and $\xi \in H_{m}^{\bar{q}}(X ; R)$, where $\Phi, \phi$ are isomorphisms and the first one preserves the $\cup$-product .

Proof. We proceed in three steps.

- Construction of $\phi$. We prove that the inclusion $C_{*}^{\bar{p}}(X ; R) \stackrel{\iota}{\hookleftarrow} S_{*}(X \backslash \Sigma, R)$ is a quasi-isomorphim. We proceed as in the previous Proposition. In fact, the only item to prove is the property (3) of [14, Theorem 5.1.4]. The regular part of $\mathbb{R}^{i} \times \stackrel{\circ}{\mathrm{c}} L$ is $\left.\mathbb{R}^{i} \times\left(L \backslash \Sigma_{L}\right) \times\right] 0, \infty[$. We know that $H_{*}\left(\mathbb{R}^{i} \times\left(L \backslash \Sigma_{L}\right) \times\right] 0, \infty[; R)=H_{*}\left(L \backslash \Sigma_{L} ; R\right)$. On the other hand, we have $H_{*}^{\bar{p}}\left(\mathbb{R}^{i} \times \stackrel{\circ}{\mathrm{c}} L ; R\right)=H_{*}\left(L \backslash \Sigma_{L} ; R\right)$ (cf. [5, Prop. 5.4]). Since both isomorphisms are induced by the canonical projection then we get (3). The map $\phi$ is the isomorphism $\iota_{*}$.

- Construction of $\Phi$. Consider the operator $F$ constructed in the proof of Proposition 13.5 (b) and set $\Phi=F_{*}$.

- Diagram commutativity. As in the previous Proposition.

\section{References}

[1] M. Banagl - Rational generalized intersection homology theories, Homology, Homotopy Appl. 12 (2010), no. 1, p. 157-185.

[2] J.-P. Brasselet, G. Hector and M. Saralegi - Théorème de de Rham pour les variétés stratifiées , Ann. Global Anal. Geom. 9 (1991), no. 3, p. 211-243.

[3] G. Bredon - Topology and geometry, Graduate Texts in Mathematics, vol. 139, SpringerVerlag, New York, 1997, Corrected third printing of the 1993 original.

[4] D. Chataur, M. Saralegi-Aranguren and D. Tanré - Intersection Cohomology. Simplicial blowup and rational homotopy., ArXiv Mathematics e-prints. no. 1205.7057 (2012), To appear in Mem. Amer. Math. Soc.

[5] — Homologie d'intersection. Perversités générales et invariance topologique , ArXiv Mathematics e-prints. no. 1602.03009 (2016).

[6] — Poincaré duality with cap products in intersection homology, ArXiv Mathematics e-prints. no. 1603.08773 (2016).

[7] — Singular decompositions of a cap product, Proceedings AMS 145(2017), no. 8, p. $3645-3656$.

[8] - Steenrod squares on intersection cohomology and a conjecture of $M$ Goresky and $W$ Pardon, Algebr. Geom. Topol. 16 (2016), no. 4, p. 1851-1904.

[9] G. Friedman - Superperverse intersection cohomology: stratification (in)dependence, Math. Z. 252 (2006), no. 1, p. 49-70.

[10] _ Singular chain intersection homology for traditional and super-perversities, Trans. Amer. Math. Soc. 359 (2007), no. 5, p. 1977-2019 (electronic).

[11] — Intersection homology Künneth theorems, Math. Ann. 343 (2009), no. 2, p. 371395.

[12] — Intersection homology with general perversities, Geom. Dedicata 148 (2010), p. $103-135$ 
[13] — An introduction to intersection homology with general perversity functions, in Topology of stratified spaces, Math. Sci. Res. Inst. Publ., vol. 58, Cambridge Univ. Press, Cambridge, 2011, p. 177-222.

[14] Singular intersection homology , Available at http://faculty.tcu.edu/gfriedman/IHbook.pdf (2017).

[15] G. Friedman and J. E. McClure - Cup and cap products in intersection (co)homology, Adv. Math. 240 (2013), p. 383-426.

[16] M. Goresky and R. MacPherson - Intersection homology theory, Topology 19 (1980), no. 2, p. $135-162$

[17] _ Intersection homology. II , Invent. Math. 72 (1983), no. 1, p. 77-129.

[18] M. Goresky and P. Siegel- Linking pairings on singular spaces , Comment. Math. Helv. 58 (1983), no. 1, p. 96-110.

[19] S. Halperin- Lectures on minimal models , Mém. Soc. Math. France (N.S.) (1983), no. 9-10.

[20] M. Handel- A resolution of stratification conjectures concerning CS sets , Topology 17 (1978), no. 2, p. 167-175.

[21] H. C. King- Topological invariance of intersection homology without sheaves, Topology Appl. 20 (1985), no. 2, p. 149-160.

[22] R. MacPherson- Intersection homology and perverse sheaves , Unpublished AMS Colloquium Lectures, San Francisco (1991).

[23] M. Saralegi- Homological properties of stratified spaces, Illinois J. Math. 38 (1994), no. 1, p. $47-70$.

[24] M. Saralegi-Aranguren- de Rham intersection cohomology for general perversities, Illinois J. Math. 49 (2005), no. 3, p. 737-758 (electronic).

[25] L. C. Siebenmann- Deformation of homeomorphisms on stratified sets. I, II , Comment. Math. Helv. 47 (1972), p. 123-136; ibid. 47 (1972), 137-163.

[26] S. Willard- General topology, Addison-Wesley Publishing Co., Reading, Mass.-London-Don Mills, Ont., 1970.

Lafma, Université de Picardie Jules Verne, 33, Rue Saint-Leu, 80039 Amiens Cedex 1, FRANCE

E-mail address: David.Chataur@u-picardie.fr

Laboratoire de Mathématiques de Lens, EA 2462, Université D’Artois, SP18, Rue Jean Souvraz, 62307 Lens Cedex, France

E-mail address: martin.saraleguiaranguren@univ-artois.fr

Département de Mathématiques, UMR 8524, Université de Lille 1, 59655 Villeneuve D'AscQ Cedex, France

E-mail address: Daniel.Tanre@univ-lille1.fr 